\title{
Model structures on the category of small double categories
}

\author{
THOMAS M FIORE \\ SIMONA PAOLI \\ DORETTE PRONK
}

\begin{abstract}
In this paper we obtain several model structures on DblCat, the category of small double categories. Our model structures have three sources. We first transfer across a categorification-nerve adjunction. Secondly, we view double categories as internal categories in Cat and take as our weak equivalences various internal equivalences defined via Grothendieck topologies. Thirdly, DblCat inherits a model structure as a category of algebras over a 2 -monad. Some of these model structures coincide and the different points of view give us further results about cofibrant replacements and cofibrant objects. As part of this program we give explicit descriptions for and discuss properties of free double categories, quotient double categories, colimits of double categories, horizontal nerve and horizontal categorification.
\end{abstract}

18D05, 18G55; 55P99, 55U10

\section{Introduction}

The theory of categories enriched in Cat, called 2-categories, has been highly developed over the past 40 years and has found numerous applications. Beginning with Bénabou's bicategories (weak 2-categories) in [4], through Kelly's monograph [54] on enriched categories, and including the more recent companion by Lack [57], as well as many others, we have seen the $n=2$ case for higher category theory become very well understood. Limits in 2-categories from Kelly [53], 2-monads on 2-categories from Blackwell-Kelly-Power [8] and Kan extensions for 2-functors from Dubuc [26] are now widely known. Model structures on 2-Cat have also been studied recently by Lack [58; 59] and Worytkiewicz-Hess-Parent-Tonks [83]. Model structures, more generally, have been used in the study of $(\infty, 1)$-categories as a means of comparison by Bergner [5; 6; 7], Joyal-Tierney [52], Rezk [75] and Toën [81].

Recent examples, however, show that 2-categories are not enough and that one must invoke Ehresmann's earlier notion of double category [30; 31]. In many mathematical situations one is interested in two types of morphisms, which may or may not interact. Between rings, for example, there are ring homomorphisms as well as bimodules. 
Between manifolds there are differentiable maps and cobordisms, which are both used in field theory. Between categories there are functors as well as adjunctions. The notion of 2-category does not capture both types of morphisms, but the notion of (pseudo) double category certainly does. ${ }^{1}$

Concisely, a small double category is an internal category in Cat. A small double category consists of a set of objects, a set of horizontal morphisms, a set of vertical morphisms and a set of squares, equipped with various associative and unital compositions satisfying the interchange law. In addition to the early work of Bastiani-Ehresmann [2], Ehresmann-Ehresmann [27; 28; 29], C Ehresmann [30; 31] and Brown-Spencer [18], recent work on double categories has been completed by Brown and collaborators [15; 16; 17], Dawson-Paré [20; 21; 22], Dawson-Paré-Pronk [23], Fiore [34], Garner [38], Grandis-Paré [42; 43; 44; 45], Kock [56], Shulman [76; 77] and others.

Double categories are the $n=2$ case for $n$-fold categories, which have been studied and applied for some time now. In the same way that higher categories may be defined by iterated enrichment, one may define wider categories or $n$-fold categories via iterated internalization. The edge symmetric ${ }^{2}$ case has been studied by Brown and Higgins in the concept of cubical $\omega$-category and by Grandis in the concept of symmetric weak cubical categories in [40] and [41]. Further, $n$-fold categories internal to the category of groups have been used to model connected homotopy $(n+1)$-types by Loday [63] as summarized in the survey paper by Paoli [70]. Recent work includes Lack-Paoli [61] and Paoli [71]. Applications of versions of the $n=2$ case of internalized categories include Dawson-Paré-Pronk [25], Fiore [33; 34], Kerler-Lyubashenko [55], MaySigurdsson [66], Morton [69] and Shulman [76; 77]. Thus, there has been a general trend towards $n$-fold categories, especially the $n=\omega$ and $n=2$ cases.

In this article we introduce model categories into the theory of double categories, anticipating a utility in the theory of wider categories analogous to that of model structures in the theory of higher categories. Already in the $n=2$ case we see that $n$-fold categories and $n$-categories diverge: even though the homotopy theory of 2-categories resembles that of categories, the homotopy theory of double categories is much richer. This results from the numerous ways to view a double category: as an internal category in Cat, as a categorical structure with two directions, as certain simplicial objects in Cat, as certain bisimplicial sets or as algebras over a 2-monad.

\footnotetext{
${ }^{1}$ These examples and others can be found in the article of Grandis-Paré [42]. There it is remarked in the introduction: "We conclude with some remarks about the motivation of this work. Its leitmotif can be summarised as follows: arrows which are too relaxed (like profunctors, spans, relations) or too strict (like adjunctions) to have limits, can be studied in a (pseudo) double category, correlating them with more ordinary (horizontal) arrows."

${ }^{2}$ Edge symmetric means that the $n$-morphisms in all $n+1$ directions are the same.
} 
Each point of view suggests different notions of weak equivalence and fibration. The new types of pasting diagrams available in a double category also create new phenomena. We take these various points of view into consideration when constructing the model structures.

Thus, our model structures have three sources. First, we transfer the Thomason diagram structure and categorical diagram structure on the category of simplicial objects in Cat to DblCat via a horizontal categorification-horizontal nerve adjunction. In the Thomason structure on Cat in [80], a functor is a weak equivalence if and only if its nerve is a weak homotopy equivalence of simplicial sets. In the categorical structure on Cat of Joyal-Tierney [51] (reproved by Rezk in [74]), a functor is a weak equivalence if and only if it is an equivalence of categories. Both the Thomason structure and the categorical structure on Cat are cofibrantly generated, and thus induce cofibrantly generated model structures on simplicial objects in Cat where weak equivalences and fibrations are defined levelwise. We apply Kan's Lemma on Transfer of cofibrantly generated model structures (Theorem 7.11) to transfer both of these diagram structures to DblCat (Theorem 7.13 and Theorem 7.17). However, the application is not straightforward, and we must make several double categorical preparations, including horizontal categorification and a pushout formula in DblCat. We also prove one negative result in Theorem 7.22: it is impossible to transfer the Reedy categorical structure on $\mathbf{C a t}^{\Delta^{\mathrm{op}}}$ to DblCat. The transfer from bisimplicial sets is treated by Fiore-Paoli in [35]. Even better, [35] contains a Thomason model structure on the category of small $n$-fold categories, and this model structure is Quillen equivalent to SSet.

We arrive at a second source for model structures on DblCat when we view double categories as internal categories in Cat. In this way we obtain double categorical versions of the categorical structure on Cat, where a functor is a weak equivalence if and only if it is fully faithful and essentially surjective. Although the notion of fully faithfulness makes sense internally, essential surjectivity does not, and therefore equivalences of internal categories need further explanation. Model structures on categories internal to a good category $\mathbf{C}$ have already been developed by EveraertKieboom-Van der Linden in [32], and we apply their results to the case $\mathbf{C}=$ Cat. They define essential surjectivity (and hence also weak equivalences) with respect to a Grothendieck topology $\mathcal{T}$ on $\mathbf{C}$. We take simplicially surjective functors and categorically surjective functors as bases for Grothendieck topologies on Cat, and obtain two distinct model structures in Sections 8.2 and 8.3. Additionally, we show in Theorem 8.52 that the model structure induced by the trivial topology coincides with the trivial model structure from the 2-category of internal categories. 
Third, DblCat inherits a model structure as a category of algebras over a 2-monad. This is an application of Lack's algebra structure [60]. The underlying 1-category of a 2-category with finite limits and finite colimits always admits the so-called trivial model structure, whose weak equivalences are equivalences and fibrations are isofibrations. If $\mathcal{K}$ is a locally finitely presentable 2 -category equipped with a $2-\operatorname{monad} T$ with rank, then the category of (strict) $T$-algebras is a model category: a morphism of $T$-algebras is a weak equivalence or fibration if and only if its underlying morphism is a weak equivalence or fibration in the trivial model structure on $\mathcal{K}$. In our application of [60] in Section 9, $\mathcal{K}$ is the 2-category Cat(Graph) of internal categories in small nonreflexive graphs, and $T$ is the $2-$ monad $\bar{M}$ induced by the Cartesian monad $M$ on Graph whose algebras are categories.

Depending on the reader's experience, certain model structures will be of more interest than others. Simplicially-minded readers will no doubt find the transfers from $\mathbf{C a t}^{\Delta^{\mathrm{op}}}$ most interesting, while categorically-minded readers may find the model structures on Cat(Cat) arising from [32] more interesting. Universal algebraists may find the third point of view most appealing, namely double categories as algebras for a 2-monad. Nevertheless, certain model structures can be defined from two points of view, and will thus be of interest to readers working in different fields.

In other words, we prove that some of these model structures coincide. The model structure obtained by transferring the categorical diagram structure across the vertical categorification-vertical nerve adjunction is the same as the model structure associated to the simplicially surjective topology on Cat (Corollary 8.29). The algebra structure is the same as the model structure associated to the categorically surjective topology on Cat (Theorem 9.1).

These two different constructions of the same model structures yield more refined information about cofibrant replacements and cofibrant objects. For example, the cofibrant objects in the algebra structure are known to be precisely the flexible algebras, but from the categorically-surjective-topology structure we see that the flexible double categories are precisely those with object category free on a graph (Corollary 9.4 and Remark 9.7). Such a description allows us to conclude that the flexible 2-categories of Lack [58] are indeed flexible algebras for a 2-monad. Lack's Theorem 4.8 (iv) in [58], which characterizes flexible 2-categories as those 2-categories with underlying 1 -category a free category on a graph, now extends to double categories.

We also compare our model structures on DblCat with the analogous ones for Cat in Propositions 7.16, 7.20 and 8.56. The vertical embedding of Cat into DblCat preserves and reflects weak equivalences, fibrations and cofibrations from the Thomason structure into the transferred diagram Thomason structure, as well as from the categorical 
structure into the transferred diagram categorical structure. The horizontal embedding of Cat into DblCat preserves and reflects weak equivalences, fibrations and cofibrations from the categorical structure into the trivial structure. However, the vertical inclusion of 2-Cat into DblCat preserves neither the weak equivalences nor the cofibrations of the categorical structure into the algebra structure, as shown at the end of Section 9. Nevertheless, a 2-category is cofibrant in 2-Cat if and only if its vertical embedding into DblCat is cofibrant.

In order to build our model structures we prove various general results about double categories, so far not available in the literature. These results are also of independent interest for the theory of double categories in its own right. We develop free double categories, their quotients and colimits of double categories using a double categorical version of Street's 2-categorical notion of derivation scheme [78]. In particular we obtain an explicit formula for two pushouts of double categories in Theorem 10.6, which is essential for our application of Kan's Lemma on Transfer in Theorem 7.13 and Theorem 7.17. We also prove that the 2-categories DblCat $\mathbf{v}$ and DblCat $_{\mathbf{h}}$ are 2-cocomplete in Theorem 4.2.

Free double categories on reflexive double graphs have been studied by Dawson-Paré in [22]. By reflexive double graph we mean a collection of objects, vertical edges, horizontal edges and squares equipped with source and target maps, identity edges and identity squares. In this paper, we will instead use double graphs with 1-identities. A double graph with 1-identities is like a reflexive double graph, except identity squares are not required. Between double graphs with 1-identities and double categories, there is the intermediate notion of double derivation scheme. A double derivation scheme is a double graph with 1-identities in which the horizontal and vertical reflexive 1-graphs are categories. In the free double category on a double derivation scheme, the vertical and horizontal 1-categories are preserved, but nontrivial squares consist of allowable compatible arrangements. Since we are considering compatible arrangements of squares in a double derivation scheme rather than in a double reflexive graph, our allowable compatible arrangements are different than the composable compatible arrangements of Dawson-Paré [21].

Free double categories on double derivation schemes and their quotients allow us to construct colimits of double categories. First one takes the colimits of the vertical and horizontal 1-categories. These, together with the colimit of the sets of squares, form a double derivation scheme. Finally, we mod out the free double category on this double derivation scheme by the smallest congruence which guarantees that the natural maps are double functors, and the result is the colimit in DblCat. This colimit formula is the basis of Theorem 10.6 which gives an explicit description of the pushouts of a double functor along two inclusions of external products. This theorem is crucial for 
our application of Kan's Lemma on Transfer. These two pushouts are special cases of a more general theorem on pushouts along inclusions of external products, which will appear in a separate paper with a comparison to Dawson-Paré-Pronk [24].

Free double categories on double derivation schemes and their quotients find further application in the construction of fundamental double categories of simplicial objects in Cat, that is, in our construction of a left adjoint to the horizontal nerve. We obtain an important example of our explicit constructions of fundamental double categories in a second way as well, namely via weighted colimits (see Example 6.6 and Proposition $6.11)$.

We begin in Section 2 with a review of double categories, including horizontal 2categories, vertical 2-categories, double functors, horizontal and vertical natural transformations, the external product of 2-categories and Cartesian closedness of the category DblCat, as well as the 2-categories DblCat $\mathbf{v}_{\mathbf{v}}$ and DblCat $_{\mathbf{h}}$. Free double categories on double derivation schemes are introduced in Section 3 and are used in Section 4 to describe colimits in DblCat. The horizontal nerve is discussed in Section 5 along with its representable definition in terms of external products of finite ordinals. In Section 6, free double categories on double derivation schemes and their quotients are applied to construct the left adjoint to the horizontal nerve. Section 7 focuses on transferring model structures across the horizontal categorification-horizontal nerve adjunction and recalls model structures on Cat, smallness issues and Kan's Lemma on Transfer. Section 8 begins with an exposition of the methods of EveraertKieboom-Van der Linden [32], and then applies them to obtain model structures on Cat $($ Cat $)=$ DblCat induced by three Grothendieck topologies on Cat: the simplicially surjective topology, the categorically surjective topology, and the trivial topology. The model structure induced by the simplicially surjective topology coincides with the transfer of the diagram categorical structure across the adjunction $c_{v} \dashv N_{v}$. In Section 9 we prove that the algebra structure on DblCat coincides with the model structure induced by the categorically surjective topology. In Section 10, the Appendix, we obtain an explicit description of certain pushouts in DblCat, namely Theorem 10.6. We use this to characterize the behavior of the horizontal nerve on such pushouts in Theorem 10.7. The essential application is to pushouts along the generating acyclic cofibrations in the transfer in Section 7.

Acknowledgements Thomas M Fiore was supported by National Science Foundation Grant DMS 0501208 at the University of Chicago. At the Universitat Autònoma de Barcelona he was supported by Grant SB2006-0085 of the Spanish Ministerio de Educación y Ciencia under the Programa Nacional de ayudas para la movilidad de profesores de universidad e investigadores españoles y extranjeros. Simona Paoli 
was supported by an Australian Research Council Postdoctoral Fellowship (project number DP0558598) and by a Macquarie University New Staff Grant Scheme. Dorette Pronk was supported by an NSERC Discovery Grant, and she also thanks Macquarie University and the University of Chicago for their hospitality and financial support, as well as Calvin College and Utrecht University for their hospitality during her sabbatical visits. All three authors gratefully acknowledge the financial support and hospitality of the Fields Institute during the Thematic Program on Geometric Applications of Homotopy Theory in 2007, at which a significant portion of this work was completed. Thomas M Fiore and Simona Paoli also thank the Centre de Recerca Matemàtica in Bellaterra (Barcelona) for its hospitality during the CRM Research Program on Higher Categories and Homotopy Theory in 2007-2008. Additional material in this article was completed during that time. Simona Paoli thanks the Universitat Autònoma de Barcelona and the Centre de Recerca Matemàtica for the financial support during her visits.

The authors thank Steve Lack for suggesting the comparison of the model structure induced by the categorically surjective topology and the algebra model structure. They also thank Michael Shulman for the simplified proof of Corollary 2.14. The authors express their gratitude to Peter May, Robert Paré and Robert Dawson for some discussion of this work. Additionally, they thank the anonymous referee for many helpful suggestions.

\section{Double categories}

We first recall the elementary notions of double category theory. In many mathematical contexts there are two interesting types of morphisms; double categories organize them into one structure. For example, between rings there are morphisms of rings as well as bimodules, between objects of any 2-category there are morphisms as well as adjunctions, and so on. Sometimes one would like to distinguish a family of squares, such as the pullback squares among the commutative squares, and double categories are also of use here. The notion of double category is not new, and goes back to Ehresmann $[30 ; 31]$.

Definition 2.1 A small double category $\mathbb{D}=\left(\mathbb{D}_{0}, \mathbb{D}_{1}\right)$ is a category object in Cat, the category of small categories. This means that $\mathbb{D}_{0}$ and $\mathbb{D}_{1}$ are small categories equipped with functors

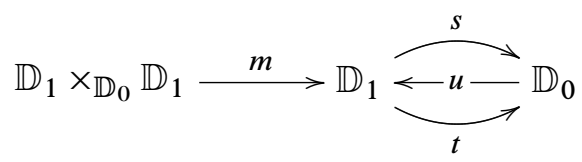


that satisfy the usual axioms of a category. We call the objects and morphisms of $\mathbb{D}_{0}$ respectively the objects and vertical morphisms of $\mathbb{D}$, and we call the objects and morphisms of $\mathbb{D}_{1}$ respectively the horizontal morphisms and squares of $\mathbb{D}$.

When one expands this definition, one sees that a small double category consists of a set of objects, a set of horizontal morphisms, a set of vertical morphisms, and a set of squares equipped with various sources, targets, and associative and unital compositions. Further, the horizontal and vertical compositions of squares are required to satisfy the interchange law. Since we only deal with small categories and small double categories, we will usually leave off the adjective small. Sources and targets are indicated as follows.

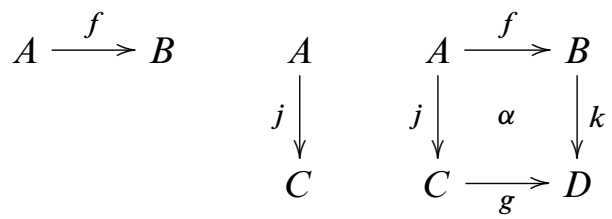

We denote the set of squares with the boundary

by

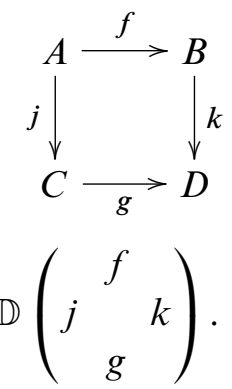

Then one has the categories

$$
(\operatorname{Obj} \mathbb{D}, \text { Hor } \mathbb{D}) \text { and }(\operatorname{Ver} \mathbb{D}, \mathrm{Sq} \mathbb{D})
$$

under horizontal composition and the categories

$$
(\operatorname{Obj} \mathbb{D}, \operatorname{Ver} \mathbb{D}) \text { and }(\text { Hor } \mathbb{D}, S q \mathbb{D})
$$

under vertical composition. We will write $\left[\begin{array}{ll}f & g\end{array}\right]$ for the horizontal composition of horizontal morphisms $f$ and $g$, and similarly $[\alpha \beta]$ for the horizontal composition of squares $\alpha$ and $\beta$. We will write $\left[\begin{array}{c}v \\ w\end{array}\right]$ for the vertical composition of vertical morphisms $v$ and $w$, and similarly $\left[\begin{array}{l}\gamma \\ \delta\end{array}\right]$ for the vertical composition of squares $\gamma$ and $\delta$.

There are many examples of double categories. The commutative squares in a given 1 -category form the squares of a double category. More generally, for a 2-category $\mathbf{C}$, 
Bastiani-Ehresmann defined the double category $\mathbb{Q} \mathbf{C}$ of quintets of $\mathbf{C}$ (pages 272 and 273 of [2]). The objects of $\mathbb{Q} \mathbf{C}$ are the objects of $\mathbf{C}$, horizontal and vertical morphisms are the morphisms of $\mathbf{C}$, and the squares $\alpha$ as in (1) are the 2-cells $\alpha: k \circ f \Longrightarrow g \circ j$. In many situations, one has examples of a slightly more general notion called pseudo double category, defined briefly in [2] on page 288 as the notion of dicategory, and defined in detail by Grandis-Paré in Section 7 of [42]. A pseudo double category is like a double category, except one direction is a bicategory (weak 2-category) rather than a 2-category. For example, the double category of rings, bimodules, ring homomorphism, and twisted maps of bimodules is weak in one direction. Another example is given by finite sets, Riemann surfaces with labelled analytically parametrized boundary components, bijections of finite sets, and holomorphic maps preserving the given structure. In these two examples we choose the horizontal direction to be weak, so that bimodules respectively Riemann surfaces are the horizontal morphisms. In this paper we work only with strict double categories, though pseudo double categories can also fit into our framework.

The notion of double category contains many familiar structures. If we view a category as an internal category in Cat with object and morphism categories discrete, it is equivalent to viewing an ordinary category as a double category with trivial vertical morphisms and trivial squares. Every 2-category $\mathbf{C}$ can be considered a double category in at least four ways: as a double category $\mathbb{H} \mathbf{C}$ with trivial vertical morphisms, as a double category $\mathbb{V} \mathbf{C}$ with trivial horizontal morphisms, as Ehresmann's quintets $\mathbb{Q} \mathbf{C}$, or as the transpose of Ehresmann's quintets $(\mathbb{Q} \mathbf{C})^{t}$. Any double category $\mathbb{D}$ has an underlying horizontal 2-category $\mathbf{H D}$ and an underlying vertical 2-category $\mathbf{V D D}$ : we obtain these substructures as the full subdouble categories with only trivial vertical morphisms or trivial horizontal morphisms respectively. We denote the underlying 1 -categories of $\mathbf{H D}$ and $\mathbf{V D}$ by $(\mathbf{H} \mathbb{D})_{0}$ and $(\mathbf{V D D})_{0}$ respectively. The subscript 0 here means underlying 1 -category of a 2 -category, and is unrelated to the subscript 0 in Definition 2.1. Though the formula $(\mathbf{V D})_{0}=\mathbb{D}_{0}$ holds, $(\mathbf{H D})_{0}$ is not the same as $\mathbb{D}_{0}$.

Definition 2.2 A double functor $F: \mathbb{D} \longrightarrow \mathbb{E}$ is an internal functor in Cat. Such a functor consists of functions

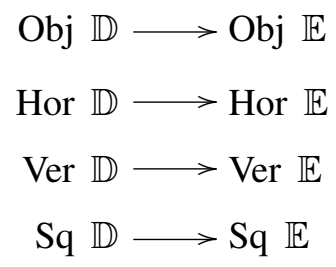

which preserve all sources, targets, compositions, and identities. 
Internal natural transformations in Cat are also called horizontal natural transformations. We recall the notion from pages 249 and 250 of Bastiani-Ehresmann's article [2], or 1.4 of Grandis-Paré's article [42].

Definition 2.3 If $F, G: \mathbb{D} \longrightarrow \mathbb{E}$ are double functors, then a horizontal natural transformation $\theta: F \Longrightarrow G$ as in [42] assigns to each object $A$ a horizontal morphism $\theta A: F A \longrightarrow G A$ and assigns to each vertical morphism $j$ a square

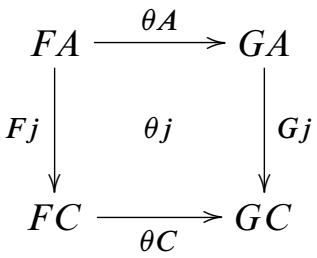

such that:

(1) For all $A \in \mathbb{D}$, we have $\theta 1_{A}^{v}=i_{\theta A}^{v}$.

(2) For composable vertical morphisms $j$ and $k$,

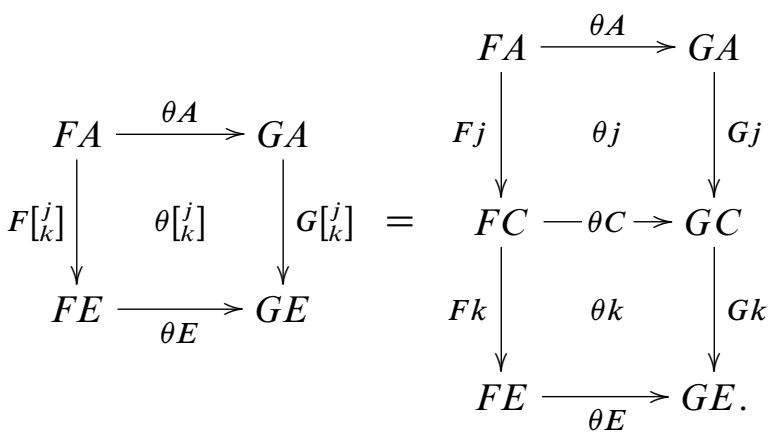

(3) For all $\alpha$ as in Diagram (1),

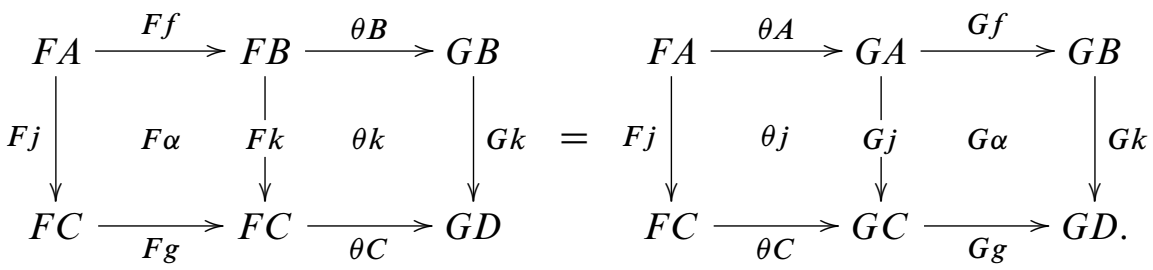

We also need the analogous notion of vertical natural transformation. 
Definition 2.4 If $F, G: \mathbb{D} \longrightarrow \mathbb{E}$ are double functors, then a vertical natural transformation $\sigma: F \Longrightarrow G$ as in [42] assigns to each object $A$ a vertical morphism $\sigma A: F A \longrightarrow G A$ and assigns to each horizontal morphism $f$ a square

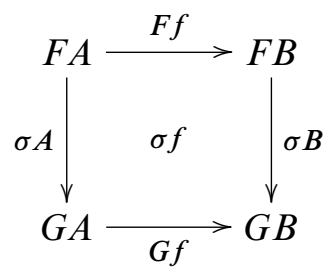

such that:

(1) For all objects $A \in \mathbb{D}$, we have $\sigma 1_{A}^{h}=i_{\sigma A}^{h}$.

(2) For all composable horizontal morphisms $f$ and $g$,

$$
\sigma[f g]=\left[\begin{array}{ll}
\sigma f & \sigma g
\end{array}\right] .
$$

(3) For all $\alpha$ as in Diagram (1),

$$
\left[\begin{array}{c}
F \alpha \\
\sigma g
\end{array}\right]=\left[\begin{array}{l}
\sigma f \\
G \alpha
\end{array}\right] .
$$

Thus, double categories form a 2-category in two different ways, depending on the choice of 2-cell. Further, there are useful adjunctions with 2-Cat and Cat. Our notation, introduced before Definition 2.2, follows that found in Section 1.3 of [42].

Proposition 2.5 (Compare page 80 of [29].) Let DblCat $_{h}$ respectively DblCat $_{\mathbf{v}}$ denote the 2-categories of small double categories, double functors, and horizontal natural transformations respectively vertical natural transformations. Let 2-Cat denote the 2-category of small 2-categories, 2-functors, and 2-natural transformations. ${ }^{3}$ Then the inclusion 2-functors

$$
\begin{aligned}
& \mathbb{H}: \text { 2-Cat } \longrightarrow \text { DblCat }_{\text {}} \\
& \mathbb{V}: \text { 2-Cat } \longrightarrow \text { DblCat }_{\mathbf{v}}
\end{aligned}
$$

have as right 2 -adjoints the 2 -functors

$$
\begin{aligned}
& \text { H: } \text { DblCat }_{h} \longrightarrow \text { 2-Cat } \\
& \text { V: } \text { DblCat }_{\mathbf{v}} \longrightarrow \text { 2-Cat }
\end{aligned}
$$

\footnotetext{
${ }^{3}$ In this article we follow the convention that 2 -functors and 2 -natural transformations are strict 2-functors and strict 2-natural transformations.
} 
respectively. Moreover, the inclusion 2-functors

$$
\begin{aligned}
& \mathbb{H}: \text { Cat } \longrightarrow \text { DblCat }_{h} \\
& \mathbb{V}: \text { Cat } \longrightarrow \text { DblCat }_{\mathbf{v}}
\end{aligned}
$$

have as right 2 -adjoints the 2 -functors

$$
\begin{aligned}
& (\mathrm{H}-)_{0}: \text { DblCat }_{\mathbf{h}} \longrightarrow \text { Cat } \\
& (\mathrm{V}-)_{0}: \text { DblCat }_{\mathbf{v}} \longrightarrow \text { Cat }
\end{aligned}
$$

respectively.

The external product of 1-categories was defined by Bastiani-Ehresmann on page 251 of [2], and called there the square product. We extend this notion to 2-categories in Definition 2.6, and even double categories in Remark 2.8.

Definition 2.6 If $\mathbf{C}$ and $\mathbf{D}$ are 2-categories, then their external product $\mathbf{C} \otimes \mathbf{D}$ is the double category with objects $\operatorname{Obj} \mathbf{C} \times \operatorname{Obj} \mathbf{D}$,

vertical morphisms $(j, D):(C, D) \longrightarrow\left(C^{\prime}, D\right)$,

horizontal morphisms $(C, f):(C, D) \longrightarrow\left(C, D^{\prime}\right)$,

and squares

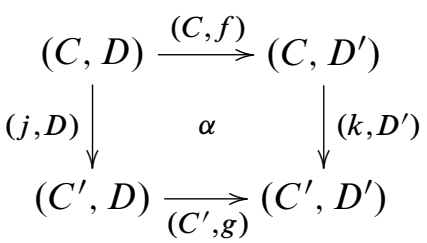

given by pairs $\alpha=(\gamma, \delta)$ of 2-cells $\gamma: j \Longrightarrow k$ and $\delta: f \Longrightarrow g$ in $\mathbf{C}$ and $\mathbf{D}$ respectively.

We may simplify the foregoing definitions using the operation of transposition, which interchanges the roles of horizontal and vertical.

Definition 2.7 The transpose of a double category $\mathbb{D}$ is the double category $\mathbb{D}^{t}$ with

$$
\begin{aligned}
\text { Obj } \mathbb{D}^{t} & =\text { Obj } \mathbb{D} \\
\operatorname{Hor} \mathbb{D}^{t} & =\operatorname{Ver} \mathbb{D} \\
\operatorname{Ver} \mathbb{D}^{t} & =\operatorname{Hor} \mathbb{D} \\
\mathbb{D}^{t}\left(\begin{array}{ccc} 
& f & \\
j & & k \\
& & k
\end{array}\right) & =\mathbb{D}\left(\begin{array}{lll} 
& j & \\
f & & g \\
& k
\end{array}\right)
\end{aligned}
$$


and the expected compositions and units. Transposition defines 2-functors

$$
\begin{aligned}
& (-)^{t}: \text { DblCat }_{\mathbf{h}} \longrightarrow \text { DblCat }_{\mathbf{v}} \\
& (-)^{t}: \text { DblCat }_{\mathbf{v}} \longrightarrow \text { DblCat }_{\mathbf{h}}
\end{aligned}
$$

that are mutually inverse.

Remark 2.8 A vertical natural transformation $\sigma: F \Longrightarrow G$ is a horizontal natural transformation $\sigma^{t}: F^{t} \Longrightarrow G^{t}$. The transpose of the 2-adjunction $\mathbb{H} \dashv \mathbf{H}$ is the 2 -adjunction $\mathbb{V} \dashv \mathbf{V}$. The external product of 2-categories $\mathbf{C}$ and $\mathbf{D}$ is $\mathbf{C} \otimes \mathbf{D}=$ $\mathbb{V} \mathbf{C} \times \mathbb{H} \mathbf{D}=(\mathbb{H} \mathbf{C})^{t} \times \mathbb{H} \mathbf{D}$. More generally, the external product of double categories $\mathbb{C}$ and $\mathbb{D}$ is $\mathbb{C} \otimes \mathbb{D}:=\mathbb{C}^{t} \times \mathbb{D}$.

Lemma 2.9 The external product of 2-categories is a functor

\section{$\otimes:$ 2-Cat $\times$ 2-Cat $\longrightarrow$ DblCat .}

Proof Transpose is functorial.

Example 2.10 Let $[m]$ denote the partially ordered set $\{0,1,2, \ldots, m\}$. Then the double category $[m] \otimes[n]$ has the shape

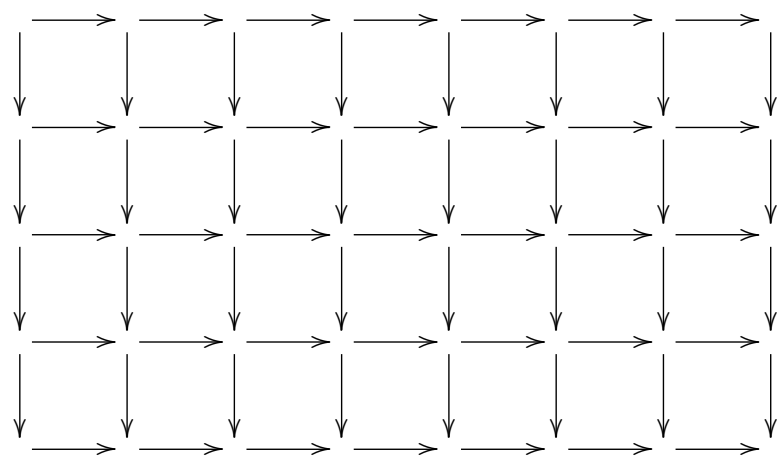

with $m$ rows and $n$ columns of squares.

We round off this section with a discussion of Cartesian closedness for DblCat, DblCat $_{v}$, and DblCat . $_{\text {. }}$.

Proposition 2.11 ( $n=2$ case of Ehresmann-Ehresmann [28]) The category DblCat is Cartesian closed. In other words for each $\mathbb{D}$ there is an endofunctor $(-)^{\mathbb{D}}$ of DblCat and a bijection of sets

$$
\operatorname{DblCat}(\mathbb{C} \times \mathbb{D}, \mathbb{E}) \cong \operatorname{DblCat}\left(\mathbb{C}, \mathbb{E}^{\mathbb{D}}\right)
$$


natural in $\mathbb{C}$ and $\mathbb{E}$.

Corollary 2.12 (pages 423-425 of [28], 1.6 of [42]) The objects of $\mathbb{E}^{\mathbb{D}}$ are double functors $\mathbb{D} \longrightarrow \mathbb{E}$, horizontal morphisms are horizontal natural transformations, vertical morphisms are vertical natural transformations, and squares are modifications.

Proof In Equation (2), we take $\mathbb{C}$ to be the terminal double category, $\mathbb{H}[1], \mathbb{V}[1]$, or $[1] \otimes[1]$. See 1.6 of [42] for the definition of modification.

Proposition 2.13 The 2-category DblCat is $_{\mathbf{v}}$ Cartesian closed. More precisely, the functor $(-)^{\mathbb{D}}$ of Proposition 2.11 and Corollary 2.12 extends to an endo-2-functor of DblCat $_{\mathbf{v}}$, and there is an isomorphism of categories

$$
\operatorname{DblCat}_{\mathbf{v}}(\mathbb{C} \times \mathbb{D}, \mathbb{E}) \cong \operatorname{DblCat}_{\mathbf{v}}\left(\mathbb{C}, \mathbb{E}^{\mathbb{D}}\right)
$$

2-natural in $\mathbb{C}$ and $\mathbb{E}$. Similarly, the 2-category DblCat $_{\mathbf{h}}$ is Cartesian closed.

Proof Equation (2) is the object part of the isomorphism in Equation (3). For the bijection of morphism sets we have

$$
\begin{aligned}
\operatorname{Mor}_{\operatorname{DblCat}}(\mathbb{C} \times \mathbb{D}, \mathbb{E}) & \cong \operatorname{DblCat}(\mathbb{V}[1] \times \mathbb{C} \times \mathbb{D}, \mathbb{E}) \\
& \cong \operatorname{DblCat}\left(\mathbb{V}[1] \times \mathbb{C}, \mathbb{E}^{\mathbb{D}}\right) \\
& \cong \operatorname{Mor}_{\operatorname{DblCat}}\left(\mathbb{C}, \mathbb{E}^{\mathbb{D}}\right)
\end{aligned}
$$

The proof that this isomorphism of graphs is a 2-natural functor is similar to the analogous proof of the Cartesian closedness of Cat.

Corollary 2.14 For a small category $\mathbf{C}$ and small double categories $\mathbb{D}$ and $\mathbb{E}$, we have an isomorphism of categories

$$
\operatorname{DblCat}_{\mathbf{v}}(\mathbb{V} \mathbf{C} \times \mathbb{D}, \mathbb{E}) \cong \operatorname{Cat}\left(\mathbf{C}, \operatorname{DblCat}_{\mathbf{v}}(\mathbb{D}, \mathbb{E})\right)
$$

2-natural in $\mathbf{C}$ and $\mathbb{E}$.

Proof From Equation (3) and the 2-adjunction $\mathbb{V} \dashv(\mathbf{V}-)_{0}$ of Proposition 2.5 we have a 2-natural isomorphism of categories

$$
\begin{aligned}
\operatorname{DblCat}_{\mathbf{v}}(\mathbb{V} \times \mathbb{D}, \mathbb{E}) & \cong \operatorname{DblCat}_{\mathbf{v}}\left(\mathbb{V} \mathbf{C}, \mathbb{E}^{\mathbb{D}}\right) \\
& \cong \operatorname{Cat}\left(\mathbf{C},\left(\mathbf{V}\left(\mathbb{E}^{\mathbb{D}}\right)\right)_{0}\right) \\
& \cong \operatorname{Cat}\left(\mathbf{C}, \operatorname{DblCat}_{\mathbf{v}}(\mathbb{D}, \mathbb{E})\right)
\end{aligned}
$$




\section{Free double categories and quotients}

As expected, there is a notion of free double category and quotient double category. However, the situation is richer than for ordinary categories, as there is an intermediate step between double categories and double graphs, which we call double derivation schemes. Double derivation schemes and quotients are crucial in the explicit description of colimits in Section 4, the construction of a left adjoint to horizontal nerve in Section 6, and the computation of pushouts in Theorem 10.6 and Theorem 10.7.

In this section we introduce double analogues to some of Street's concepts in [78]. The special kind of double graphs we will work with have 1-identities but are not equipped with identity squares. This is important because nontrivial squares in a double category may very well have one or more trivial edges. Recall that a reflexive graph is a graph equipped with a distinguished identity edge $1_{A}: A \longrightarrow A$ for each vertex $A$. All graphs in this paper are directed and small, so we often leave off the adjectives directed and small.

Definition 3.1 A double graph $\mathbb{A}$ is an internal graph in the category of small graphs. This consists of a set of vertices (objects) Obj $\mathbb{A}$, a set of horizontal edges Hor $\mathbb{A}$, a set of vertical edges Ver $\mathbb{A}$, and a set of squares $\mathrm{Sq} \mathbb{A}$ equipped with source and target maps as in Diagram (1). A morphism of double graphs is a morphism of internal graphs in the category of small graphs, or equivalently, a map which preserves the sources and targets of Diagram (1). We denote the horizontal and vertical 2-graphs of a double graph $\mathbb{A}$ by $\mathbf{H A}$ and $\mathbf{V A}$.

Definition 3.2 A double graph with 1-identities is a double graph in which the horizontal and vertical 1-graphs are reflexive graphs. This means for each object $A$, there is a distinguished horizontal edge $1_{A}^{h}: A \longrightarrow A$ as well as a distinguished vertical edge $1_{A}^{v}: A \longrightarrow A$. There are no distinguished squares. A morphism of double graphs with 1-identities is a morphism of double graphs which preserves the distinguished edges. Double graphs with 1 -identities form a category which we denote by DblGr1-Id.

A double graph with 1-identities is a double category without any of the compositions and without identity squares. The intermediate structure between double graphs with 1 -identities and double categories is analogous to Street's notion of derivation scheme in [78].

Definition 3.3 A double derivation scheme is a double graph with 1-identities whose vertical reflexive 1-graph and horizontal reflexive 1-graph are categories. A morphism 
of double derivation schemes is a morphism of double graphs with 1-identities which is a functor on both the horizontal and vertical 1-categories. Double derivation schemes form a category which we denote by DbIDerSch. We denote the horizontal and vertical derivation schemes of a double derivation scheme $\mathbb{S}$ by $\mathbf{H S}$ and $\mathbf{V S}$, and their underlying categories by $(\mathbf{H S})_{0}$ and $(\mathbf{V S})_{0}$.

To take a free category on a reflexive graph, one merely takes paths of composable edges and identifies paths which differ only by insertion or deletion of identity edges. However, the 2-dimensional situation is more subtle, as evidenced by Dawson-Paré [22], Johnson [49] and Power [72; 73]. Thus, in the construction of a free double category we need a careful definition of allowable compatible arrangement. We use the notion of compatible arrangement from Dawson-Paré [21], and develop it further for our purposes.

Definition 3.4 In a double derivation scheme $\mathbb{S}$, a compatible arrangement consists of a subdivision of a rectangle into smaller rectangles and a function which assigns to each vertex an object, to each horizontal line segment a horizontal morphism, to each vertical line segment a vertical morphism, and to each constituent rectangle a square in $\mathbb{S}$, which are compatible in the following sense:

(1) For each horizontal edge in the subdivision, the domain and codomain respectively of the morphism assigned to it are the objects assigned to the left and right vertices respectively.

(2) For each vertical edge in the subdivision, the domain and codomain respectively of the morphism assigned to it are the objects assigned to the top and bottom vertices respectively.

(3) For each constituent rectangle the composition of the morphisms assigned to the edges on

(a) the left side is the horizontal domain of the square assigned to it;

(b) the right side is the horizontal codomain of the square assigned to it;

(c) the top is the vertical domain of the square assigned to it;

(d) the bottom is the vertical codomain of the square assigned to it.

In the free double category on a double derivation scheme, a square is a compatible arrangement for which the image under any morphism of double derivation schemes into any double category becomes composable to a single square by a sequence of horizontal and vertical compositions. We will call such compatible arrangements allowable. However, an image of a compatible arrangement is just a compatible arrangement in the target double category with the same underlying subdivision of 
the rectangle. So whether a compatible arrangement is allowable in the free double category depends only on its shape, ie, the underlying subdivision of the rectangle.

A horizontal (respectively vertical) cut in a compatible arrangement is a horizontal (respectively vertical) line segment which consists of edges of the underlying subdivision of the rectangle. A horizontal (respectively vertical) cut is full length if it stretches from the left (respectively top) edge of the arrangement to the right (respectively bottom) edge of the arrangement. We can use this notion to characterize when a compatible arrangement is allowable.

Definition 3.5 A subdivision of a rectangle is allowable if it is either the trivial subdivision, consisting of just the rectangle itself, or contains a full length horizontal or vertical cut which divides it into two allowable subdivisions. A compatible arrangement is allowable if its underlying subdivision of the rectangle is allowable.

As an illustration, consider the following two examples of subdivisions of a rectangle.

Allowable:

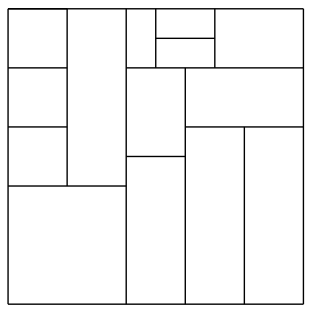

Not allowable:

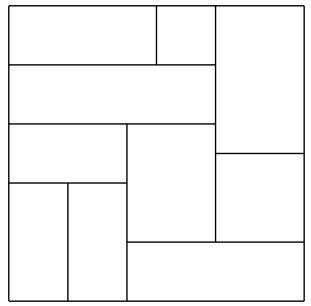

Note that our notion of allowable compatible arrangement differs from Dawson-Paré's notion of composable compatible arrangement [21] in that a compatible arrangement in a double category $\mathbb{D}$ is composable if it is composable to a single square through the use of compositions in $\mathbb{D}$ and factorizations. So their notion depends on the ambient double category, not only on the shape of the arrangement. Any allowable compatible arrangement in our sense is composable in the sense of Dawson-Paré.

Proposition 3.6 A compatible arrangement in a double category is allowable if and only if it can be composed to a single square by a sequence of horizontal and vertical compositions.

Proof We argue by induction on the number of squares in the arrangement. The statement is trivially true for arrangements consisting of a single square. Now let $C A$ be a compatible arrangement consisting of two or more squares, with an assignment into a double category $\mathbb{D}$ which is composable by a sequence of horizontal and vertical 
compositions of squares. Consider the last composition used. Without loss of generality, assume that this is a horizontal composition of two squares $\gamma_{1}$ and $\gamma_{2}$ along a vertical morphism $v$, as below.

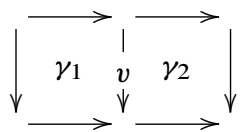

Both $\gamma_{1}$ and $\gamma_{2}$ have been obtained by sequences of horizontal and vertical compositions of squares in $C A$, so $v$ is a vertical composition of vertical morphisms $v_{1}, \ldots, v_{n}$ in $C A$. The underlying edges of these vertical morphisms form a full length cut in the underlying subdivision of the rectangle for $C A$. The squares on the left side of this cut form a compatible arrangement, since they form a rectangular subset of a compatible arrangement. Call this arrangement $C A_{1}$. It can be composed to $\gamma_{1}$ by a subsequence of the horizontal vertical compositions used for $C A$. In the same way, the squares on the right side of this cut form a compatible arrangement $\mathrm{CA}_{2}$ which can be composed to $\gamma_{2}$ by a sequence of horizontal and vertical compositions. Since both $C A_{1}$ and $C A_{2}$ contain strictly less squares than $C A$, the induction hypothesis gives that they are both allowable compatible arrangements.

Conversely, suppose that a compatible arrangement $C A$ of two or more squares in a double category $\mathbb{D}$ is allowable. Then it contains a horizontal (respectively vertical) cut into two allowable compatible arrangements $C A_{1}$ and $C A_{2}$. By induction these arrangements can be composed to single squares in $\mathbb{D}$ by sequences of horizontal and vertical compositions. Now consider the sequence of horizontal and vertical compositions used for $C A_{1}$ followed by the one for $C A_{2}$ and then one final vertical (respectively horizontal) composition along the cut. This shows that $C A$ is composable to a single square in $\mathbb{D}$ by a sequence of horizontal and vertical compositions of squares.

For inductive arguments on the number of squares in an allowable compatible arrangement, we need to know that cutting an allowable arrangement along any full length cut produces two smaller allowable compatible arrangements.

Proposition 3.7 If $C A$ is a compatible arrangement which is allowable, then any full length cut divides the arrangement into two allowable compatible arrangements.

Proof We prove this by induction on the number of squares in the arrangement. It is obviously true for compatible arrangements consisting of a single square. For an arrangement consisting of $n \geq 2$ squares, let $\mathcal{C}_{1}$ be an arbitrary full length cut as in this proposition and let $\mathcal{C}_{2}$ be the full length cut used to establish that $C A$ is allowable. Assume without loss of generality that $\mathcal{C}_{2}$ is horizontal. Let $C A_{1}$ and $C A_{2}$ be the 


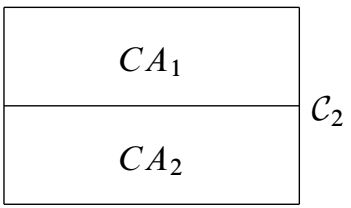

Figure 1: $C A$ cut along $\mathcal{C}_{2}$

compatible arrangements obtained by cutting $C A$ along $\mathcal{C}_{2}$, as in Figure 1 . Note that both of the arrangements $C A_{1}$ and $C A_{2}$ are allowable and contain strictly less than $n$ squares.

If $\mathcal{C}_{1}$ is vertical, the cut $\mathcal{C}_{1}$ itself gets divided by $\mathcal{C}_{2}$ into two vertical cuts $\mathcal{C}_{1,1}$ and $\mathcal{C}_{1,2}$, which are full length vertical cuts for $C A_{1}$ and $C A_{2}$ respectively, as in Figure 2. The cut $\mathcal{C}_{1,1}$ divides $C A_{1}$ into compatible arrangements $C A_{1,1}$ and $C A_{1,2}$, and the

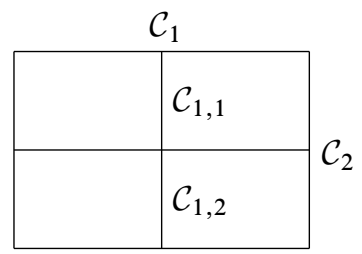

Figure 2: Vertical $\mathcal{C}_{1}$ cut by $\mathcal{C}_{2}$ into $\mathcal{C}_{1,1}$ and $\mathcal{C}_{1,2}$

cut $\mathcal{C}_{1,2}$ divides $C A_{2}$ into compatible arrangements $C A_{2,1}$ and $C A_{2,2}$, as in Figure 3 . By the induction hypothesis, $C A_{1,1}, C A_{1,2}, C A_{2,1}$, and $C A_{2,2}$ are all allowable. It

\begin{tabular}{|c|c|}
\multicolumn{1}{c}{$\mathcal{C}_{1}$} \\
\hline$C A_{1,1}$ & $C A_{1,2}$ \\
\hline$C A_{2,1}$ & $C A_{2,2}$ \\
\hline
\end{tabular}

Figure 3: $C A_{1}$ and $C A_{2}$ cut into allowable compatible arrangements

is clear that the compatible arrangement to the left of $\mathcal{C}_{1}$ gets divided into $C A_{1,1}$ and $C A_{2,1}$ by the left side of the cut $\mathcal{C}_{2}$, so the compatible arrangement to the left of $\mathcal{C}_{1}$ is allowable. In the same way the compatible arrangement to the right of $\mathcal{C}_{1}$ gets cut into 
$C A_{1,2}$ and $C A_{2,2}$ by the right side of the cut $\mathcal{C}_{2}$, so this compatible arrangement is also allowable, as we wanted to prove.

If $\mathcal{C}_{1}$ is horizontal, assume without loss of generality that $C A_{1}$ contains $\mathcal{C}_{1}$. By the induction hypothesis, $\mathcal{C}_{1}$ divides the allowable compatible arrangement $C A_{1}$ into two allowable compatible arrangements, say $C A_{1, a}$ and $C A_{1, b}$, as in Figure 4. We see that

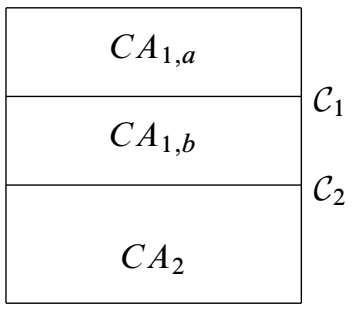

Figure 4: Horizontal $\mathcal{C}_{1}$ cuts $C A_{1}$ into allowable compatible arrangements

$\mathcal{C}_{1}$ divides the total arrangement $C A$ into two compatible arrangements, $C A_{1, a}$ and $C A_{1, c}$, the latter of which is divided by $\mathcal{C}_{2}$ into $C A_{1, b}$ and $C A_{2}$. Since both $C A_{1, b}$ and $C A_{2}$ are allowable, we conclude that both $C A_{1, c}$ and $C A_{1, a}$ are allowable. This completes the proof.

Proposition 3.8 The forgetful functors $T$ and $U$ admit left adjoints $S$ and $R$ respectively.

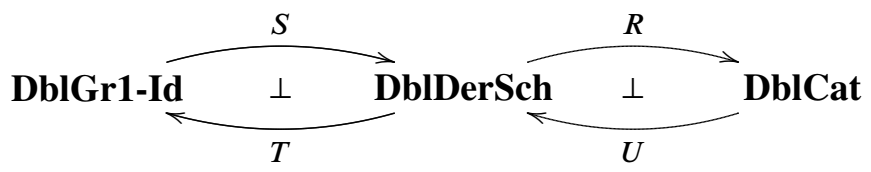

The left adjoint $S$ gives the free double derivation scheme on a double graph with 1identities, and the left adjoint $R$ gives the free double category on a double derivation scheme. The functor $R$ preserves the horizontal and vertical 1-categories.

Proof For a double graph with 1 -identities $\mathbb{A}$, let $S \mathbb{A}$ have vertical and horizontal 1 -categories the free 1-categories on the respective reflexive graphs. The set of squares remains the same. It is straightforward to verify that this defines a left adjoint to $T$.

For a double derivation scheme $\mathbb{S}$, let $R \mathbb{S}$ have vertical and horizontal 1-categories the vertical and horizontal 1-categories of $\mathbb{S}$ respectively. The squares of $R \mathbb{S}$ are allowable compatible arrangements whose constituent squares are squares of $\mathbb{S}$ and identity squares. Such compatible arrangements are composed vertically and horizontally by concatenation. Clearly, composites of allowable compatible arrangements are allowable. 
If $J: \mathbb{S} \longrightarrow U \mathbb{D}$ is a morphism of double derivation schemes, then it induces a double functor $J^{\prime}: R \mathbb{S} \longrightarrow \mathbb{D}$ which is $J$ on the horizontal and vertical 1-categories. For an allowable compatible arrangement $D$, the square $J^{\prime} D$ is the composite in $\mathbb{D}$ of $J$ applied to the constituents of $D$. Morphisms $R \mathbb{S} \longrightarrow \mathbb{D}$ restrict to morphisms $\mathbb{S} \longrightarrow U \mathbb{D}$, and it is not hard to check that these two operations are inverse. We conclude that $R \dashv U$.

Now that we have free notions, we also define quotients. Note that the notion of congruence for ordinary categories is an equivalence relation on the morphisms of highest dimension, satisfying certain compatibility properties. We imitate this in our notion of congruence for a double category.

Definition 3.9 A congruence on a category $\mathbf{C}$ is an equivalence relation on $\mathbf{C}(a, b)$ for each $a, b \in \mathbf{C}$, such that if $f \sim f^{\prime}$ and $g \sim g^{\prime}$, then $g f \sim g^{\prime} f^{\prime}$ whenever the composites exist.

Definition 3.10 A congruence on a double derivation scheme $\mathbb{S}$ consists of a congruence on the horizontal 1-category and a congruence on the vertical 1-category.

Definition 3.11 A congruence on a double category $\mathbb{D}$ consists of an equivalence relation on

$$
\mathbb{D}\left(\begin{array}{lll} 
& f & \\
j & & k \\
& g &
\end{array}\right)
$$

for each boundary

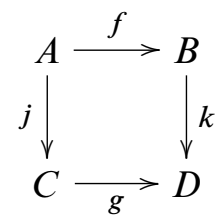

such that if $\alpha \sim \alpha^{\prime}, \beta \sim \beta^{\prime}$, and $\gamma \sim \gamma^{\prime}$ then

$$
\begin{gathered}
{\left[\begin{array}{ll}
\alpha & \beta
\end{array}\right] \sim\left[\begin{array}{ll}
\alpha^{\prime} & \beta^{\prime}
\end{array}\right]} \\
{\left[\begin{array}{l}
\alpha \\
\gamma
\end{array}\right] \sim\left[\begin{array}{l}
\alpha^{\prime} \\
\gamma^{\prime}
\end{array}\right]}
\end{gathered}
$$

whenever the composites exist. Note that the congruence does not concern the horizontal and vertical morphisms. 
Example 3.12 Suppose $K: \mathbb{D} \longrightarrow \mathbb{E}$ is a double functor. Then we may define a congruence $\sim_{K}$ on $\mathbb{D}$ by

$$
\alpha \sim_{K} \alpha^{\prime}: \Longleftrightarrow K(\alpha)=K\left(\alpha^{\prime}\right) .
$$

Proposition 3.13 Let $\mathbb{D}$ be a double category equipped with a congruence. If two allowable compatible arrangements $C A_{1}$ and $C A_{2}$ with the same underlying subdivision of the rectangle have congruent constituent squares, then the composites of $C A_{1}$ and $C A_{2}$ in $\mathbb{D}$ are congruent.

Proof By Theorem 1.2 of Dawson-Paré [21], any two composites of a composable compatible arrangement are equal. The compatible arrangements $C A_{1}$ and $C A_{2}$ are composable since they are allowable. If we compose each of $C A_{1}$ and $C A_{2}$ using the same sequence of pairwise compositions, then the pairwise composites in each step are congruent. An inductive argument shows that the total composites are then also congruent.

Definition 3.14 Let $\mathbf{C}$ be a category and $\sim$ a congruence on $\mathbf{C}$. The quotient category $\mathbf{C} / \sim$ has the same objects as $\mathbf{C}$ and has homsets $(\mathbf{C} / \sim)(a, b)=\mathbf{C}(a, b) / \sim$. The composition in $\mathbf{C} / \sim$ is induced by the composition in $\mathbf{C}$.

Definition 3.15 Let $\mathbb{S}$ be a double derivation scheme and $\sim$ a congruence on $\mathbb{S}$. The quotient double derivation scheme $\mathbb{S} / \sim$ has the same objects and squares as $\mathbb{S}$. The horizontal and vertical 1-categories of $\mathbb{S} / \sim$ are the quotient categories of $(\mathbf{H} \mathbb{S})_{0}$ and $(\mathbf{V S})_{0}$.

Definition 3.16 Let $\mathbb{D}$ be a double category and $\sim$ a congruence on $\mathbb{D}$. The quotient double category $\mathbb{D} / \sim$ has the same objects and the same horizontal and vertical 1 -categories as $\mathbb{D}$. The set of squares of $\mathbb{D} / \sim$ with the indicated boundary are

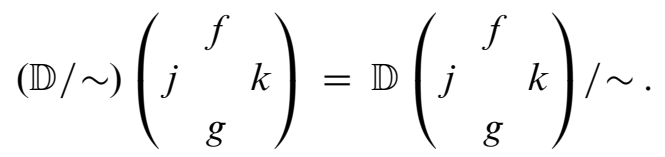

The horizontal and vertical compositions of squares in $\mathbb{D} / \sim$ are induced by the horizontal and vertical compositions of squares in $\mathbb{D}$.

These are of course not the most general notions of quotient, but more general quotients can be built from these as follows. All quotients can be characterized by the usual universal properties. 
Definition 3.17 Let $\mathbf{C}$ be a category and $\mathbf{R} \subseteq \mathbf{C} \times \mathbf{C}$ a subcategory satisfying the usual axioms of an equivalence relation both on the set of objects and on the set of morphisms. Then the quotient category $\mathbf{C} / \mathbf{R}$ is defined as follows. First we obtain a graph with object set $\mathrm{Obj} \mathbf{C} / \mathrm{Obj} \mathbf{R}$ and morphism set Mor $\mathbf{C} /$ Mor $\mathbf{R}$. We make this into a reflexive graph by identifying $1_{A}$ and $1_{B}$ whenever $A$ and $B$ are identified. Let $\mathbf{F}$ be the free category on this reflexive graph. The quotient category $\mathbf{C} / \mathbf{R}$ is defined as $\mathbf{F} / \sim$ where $\sim$ is the smallest congruence on the free category $\mathbf{F}$ such that the induced map of reflexive graphs $\mathbf{C} \longrightarrow \mathbf{F} / \sim$ is a functor.

Such quotients of categories have been considered by Boerger [9]. However a counterexample by Boerger [9] and Mersch $[67 ; 68]$ shows that the quotient functor may identify morphisms which are not equivalent. Isbell completed early work on quotients in [48]. More recently, quotients of categories by generalized congruences have been considered by Bednarczyk-Borzyszkowski-Pawlowski [3].

For general quotients of double categories, we need quotients of double derivation schemes as an intermediate notion.

Definition 3.18 Let $\mathbb{S}$ be a double derivation scheme and $\mathbb{R} \subseteq \mathbb{S} \times \mathbb{S}$ a subdouble derivation scheme satisfying the usual axioms of an equivalence relation on the sets of objects, vertical morphisms, horizontal morphisms, and squares. Then the quotient double derivation scheme $\mathbb{S} / \mathbb{R}$ is defined as follows. The horizontal and vertical 1 -categories are the quotients of the horizontal and vertical 1-categories of $\mathbb{S}$ as in Definition 3.17. The squares are $\operatorname{Sq}(\mathbb{S} / \mathbb{R})=(\operatorname{Sq} \mathbb{S}) /(\operatorname{Sq} \mathbb{R})$.

Definition 3.19 Let $\mathbb{D}$ be a double category and $\mathbb{R} \subseteq \mathbb{D} \times \mathbb{D}$ a subdouble category satisfying the usual axioms of an equivalence relation on the sets of objects, vertical morphisms, horizontal morphisms, and squares. Then the quotient double category $\mathbb{D} / \mathbb{R}$ is defined as follows. First we take the quotient of the underlying double derivation scheme of $\mathbb{D}$ by the underlying double derivation scheme of $\mathbb{R}$ as in Definition 3.18. Let $\mathbb{F}$ be the free double category $\mathbb{F}$ on this double derivation scheme. The quotient double category $\mathbb{D} / \mathbb{R}$ is defined as $\mathbb{F} / \sim$ where $\sim$ is the smallest congruence on the free double category $\mathbb{F}$ such that the induced morphism of double derivation schemes $\mathbb{D} \longrightarrow \mathbb{F} / \sim$ is a double functor.

In the above definition, see Definitions 3.11 and 3.16 for congruences and quotients of double categories by congruences. Note that only squares get identified in the last step, since the horizontal and vertical 1-categories of the free double category on a double derivation scheme are the same as the horizontal and vertical 1-categories of the double derivation scheme. 
We will make use of free double categories and their quotients in our discussion of categorification in Section 6 as well as in an explicit description of certain pushouts of double categories in Theorem 10.6 and Theorem 10.7. These are essential ingredients in the construction of model structures on DblCat. For now it is sufficient to give a colimit formula in DblCat.

\section{Limits and colimits of double categories}

Model structures in general require the existence of limits and colimits. Moreover, in order to transfer model structures along certain adjunctions we will need an explicit formula for certain pushouts of double categories, as in Theorem 10.6 and Theorem 10.7. So in this section we discuss limits and colimits of double categories. We also prove that the 2-categories DblCat $\mathbf{v}$ and DblCat $_{\mathbf{h}}$ are 2-cocomplete.

Colimits for categories were described in detail by Gabriel and Zisman. Their construction was extended by Worytkiewicz-Hess-Parent-Tonks [83] to a construction of colimits in 2-Cat. We extend this further to a construction in DblCat which goes roughly as follows. To take the colimit of a functor $F$ from an indexing category $I$ into DblCat, first we take the colimit $\mathbb{S}$ of the underlying double derivation schemes, then we take the free double category $\mathbb{F}$ on $\mathbb{S}$, and finally we form the quotient $\mathbb{F} / \sim$ of the free double category $\mathbb{F}$ by the smallest congruence $\sim$ on $\mathbb{F}$ such that the induced maps of double derivation schemes $F i \longrightarrow \mathbb{F} / \sim$ are double functors. The quotient double category $\mathbb{F} / \sim$ is the colimit of the functor $F$. The intermediate notion of double derivation scheme allows us to deal with the quotients of morphisms and quotients of squares separately. We present the details in the following theorems.

\section{Theorem 4.1 The category DblCat is complete and cocomplete.}

Proof The limits of the sets of objects, horizontal morphisms, vertical morphisms, and squares assemble to form a double category and this double category is the limit. After all, DblCat is a category of algebras.

The category DblCat is the category of models in Cat of a sketch with finite diagrams, and Cat is locally finitely presentable, so an application of Proposition 1.53 in Adámek and Rosický's book [1] shows that DblCat is locally finitely presentable. Locally finitely presentable categories are cocomplete, so DblCat is cocomplete.

Note that the underlying horizontal and vertical 2-categories of the limit are the limits of the underlying horizontal and vertical 2-categories, since $\mathbf{H}$ and $\mathbf{V}$ admit left adjoints by Proposition 2.5. The forgetful functor 2-Cat $\longrightarrow$ Cat also admits a left adjoint, so similar comments hold for the horizontal and vertical 1-categories. 
Theorem 4.2 The 2-categories DblCat $\mathbf{v}_{\mathbf{v}}$ and DblCat $_{\mathbf{h}}$ are 2-cocomplete.

Proof We prove that DblCat $_{\mathbf{v}}$ is 2-cocomplete; the statement for DblCat $_{\mathbf{h}}$ will follow, since transposition is an isomorphism of 2-categories.

The cotensor product $\{\mathbf{C}, \mathbb{E}\}$ of a category $\mathbf{C}$ with a double category $\mathbb{E}$ is $\mathbb{E}^{\mathbb{V} \mathbf{C}}$, since

$$
\begin{aligned}
\operatorname{DblCat}_{\mathbf{v}}\left(\mathbb{D}, \mathbb{E}^{\mathbb{V} \mathbf{C}}\right) & \cong \operatorname{DblCat}_{\mathbf{v}}(\mathbb{D} \times \mathbb{V} \mathbf{C}, \mathbb{E}) \\
& \cong \operatorname{DblCat}_{\mathbf{v}}(\mathbb{V} \mathbf{C} \times \mathbb{D}, \mathbb{E}) \\
& \cong \operatorname{Cat}\left(\mathbf{C}, \operatorname{DblCat}_{\mathbf{v}}(\mathbb{D}, \mathbb{E})\right)
\end{aligned}
$$

is an isomorphism of categories 2-natural in $\mathbb{D}$ by Proposition 2.13 and Corollary 2.14. Thus DblCat $\mathbf{v}_{\mathbf{v}}$ is cotensored, and by the dual of a statement on page 50 of Kelly's monograph [54], the existence of conical colimits in the 2-category DblCat $\mathbf{v}_{\mathbf{v}}$ is equivalent to the existence of ordinary conical colimits in its underlying 1-category DblCat. But ordinary conical colimits exist in DblCat by Theorem 4.1, so that the 2-category DblCat $_{\mathbf{v}}$ admits conical colimits.

The tensor product $\mathbf{C} * \mathbb{D}$ of a category $\mathbf{C}$ with a double category $\mathbb{D}$ is $\mathbb{V} \times \mathbb{D}$, since

$$
\operatorname{DblCat}_{\mathbf{v}}(\mathbb{V} \mathbf{C} \times \mathbb{D}, \mathbb{E}) \cong \operatorname{Cat}\left(\mathbf{C}, \operatorname{DblCat}_{\mathbf{v}}(\mathbb{D}, \mathbb{E})\right)
$$

is an isomorphism of categories 2-natural in $\mathbb{E}$ by Corollary 2.14. Thus DblCat is $_{\mathbf{v}}$ is tensored.

Since DblCat $_{\mathbf{v}}$ admits conical colimits and tensor products, we conclude from the dual of Theorem 3.73 in [54] that DblCat $\mathbf{v}$ is 2-cocomplete.

We work towards an explicit description of colimits in DblCat which mimics Gabriel and Zisman's calculation of colimits in Cat below.

Theorem 4.3 (Colimit formula in Cat of [37]) The colimit of a functor $F: I \longrightarrow$ Cat is calculated as follows. Let $\mathbf{F}$ be the free category on the colimit of the underlying reflexive graphs. The colimit of $F$ is the quotient $\mathbf{F} / \sim$ of the free category $\mathbf{F}$ by the smallest congruence $\sim$ on $\mathbf{F}$ such that the induced morphisms of reflexive graphs $F i \longrightarrow \mathbf{F} / \sim$ are functors.

Lemma 4.4 The horizontal and vertical 1-categories of a colimit of double derivation schemes are the colimits of the underlying horizontal and vertical 1-categories. Similarly, the horizontal and vertical 1-categories of a colimit of double categories are the colimits of the underlying horizontal and vertical 1-categories. 
Proof The right adjoint to the forgetful functor

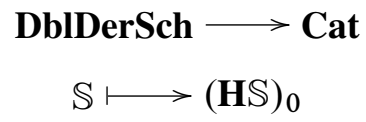

assigns to a category $\mathbf{E}$ the double derivation scheme $\mathbb{E}$ with horizontal 1-category $\mathbf{E}$, a unique vertical morphism between any two objects, and a unique square for each boundary. Similarly, the forgetful functor $\mathbb{S} \longmapsto(\mathbf{V S})_{0}$ admits a right adjoint. Since left adjoints preserve colimits, the statement for double derivation schemes follows.

The same argument works for DblCat in place of DblDerSch.

Theorem 4.5 (Colimit formula in DblDerSch) The colimit $\mathbb{S}$ of a functor

\section{$F: I \longrightarrow$ DblDerSch}

is calculated in the following way. Let $\mathbb{F}$ be the free double derivation scheme on the colimit of the underlying double graphs with 1 -identities. The colimit $\mathbb{S}$ of $F$ is the quotient $\mathbb{F} / \sim$ of the free double derivation scheme $\mathbb{F}$ by the smallest congruence $\sim$ on $\mathbb{F}$ such that the induced morphisms of double graphs with 1 -identities $F i \longrightarrow \mathbb{F} / \sim$ are morphisms of double derivation schemes.

Proof Suppose $\mathbb{S}^{\prime}$ is a double derivation scheme and $\beta_{i}: F i \longrightarrow \mathbb{S}^{\prime}$ are natural morphisms of double derivations schemes. We define a unique factorization

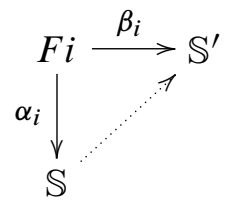

on horizontal and vertical 1-categories by the universal property of Lemma 4.4, and on squares by the universal property of the colimit of the sets Sq $F i$. The set of squares in the free double derivation scheme on a double graph with identities is the same as the set of squares in the double graph with identities by Proposition 3.8.

Theorem 4.6 (Colimit formula in DblCat) The colimit $\mathbb{C}$ of a functor

$$
F: I \longrightarrow \text { DblCat }
$$

is calculated as follows. Let $\mathbb{S}$ be the colimit in DblDerSch of the underlying double derivation schemes, and $\mathbb{F}$ the free double category on $\mathbb{S}$. The colimit $\mathbb{C}$ of $F$ is 
the quotient $\mathbb{F} / \sim$ of $\mathbb{F}$ by the smallest congruence $\sim$ such that the induced natural morphisms of double derivation schemes

$$
F i \stackrel{\alpha_{i}}{\longrightarrow} \mathbb{S} \stackrel{p}{\longrightarrow} \mathbb{F} \longrightarrow \mathbb{F} / \sim
$$

are double functors. Note that the horizontal and vertical 1 -categories of $\mathbb{S}, \mathbb{F}$, and $\mathbb{C}$ are the same, in particular the horizontal and vertical 1-categories of $\mathbb{C}$ are the colimits of the horizontal and vertical 1-categories of the Fi.

Proof Let $q: \mathbb{S} \longrightarrow \mathbb{C}$ denote the morphism of double derivation schemes defined as the composite of the inclusion $p$ with the quotient double functor from $\mathbb{F}$ to $\mathbb{C}$. Then $q \circ \alpha_{i}$ is a double functor for all $i \in I$. Suppose $\mathbb{C}^{\prime}$ is a double category and $\beta_{i}: F i \longrightarrow \mathbb{C}^{\prime}$ are natural double functors. Then by Theorem 4.5 there exists a unique morphism $J$ of double derivation schemes that makes the upper left triangle commute:

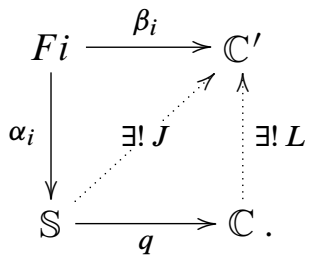

The morphism $J$ induces a double functor $K: \mathbb{F} \longrightarrow \mathbb{C}^{\prime}$ since $\mathbb{F}$ is free on $\mathbb{S}$. Since $K \circ p \circ \alpha_{i}=\beta_{i}$ is a double functor for all $i$, the induced morphisms of double derivations schemes

$$
F i \longrightarrow \mathbb{F} / \sim_{K}
$$

analogous to (4) are double functors. Here $\sim_{K}$ is defined as in Example 3.12. Since $K$ preserves $\sim_{K}$ and $\sim_{K}$ contains $\sim$, the double functor $K$ also preserves $\sim$ and induces a unique functor $L$ which makes the lower right triangle commute. Therefore the square commutes, and further $L$ is the unique double functor such that the square commutes by the uniqueness of the two fillers.

Recall that filtered colimits in Cat are particularly simple to calculate: the filtered colimit of the underlying reflexive graphs is already a category and this category is the filtered colimit in Cat. Similarly, one does not need to use free constructions and quotients to calculate filtered colimits in DblCat.

Theorem 4.7 A filtered colimit of double categories is calculated by simply taking the filtered colimits of the underlying reflexive double graphs. 
Proof The filtered colimit of the underlying reflexive double graphs admits all the associative and unital compositions necessary for a double category by the corresponding result in Cat. The interchange law holds because it is possible to find representatives of all four squares in a single stage, where the interchange law is known to hold.

\section{Nerves of double categories}

Grothendieck's full and faithful nerve $N$ : Cat $\longrightarrow$ SSet has been of tremendous use in higher category theory. One can expect that its $n$-fold version will similarly be of use. In fact, Brown-Higgins $[11 ; 12 ; 13 ; 14]$ have studied edge symmetric $n$-fold categories from the point of view of cubical sets, and also Grandis [40; 41]. A double category is a 2-truncated cubical set. We introduce in this section simplicial nerves of double categories. The simplicial nerve will be of use in Section 7 where we transfer model structures on $\mathbf{C a t}^{{ }^{\mathrm{op}}}$ to DblCat via a horizontal categorification-horizontal nerve adjunction. The bisimplicial nerve, its left adjoint, and the associated transfer of model structure, are treated in [35]. The horizontal nerve, which we consider now, is really an internal notion.

Definition 5.1 Let $\mathbb{D}=\left(\mathbb{D}_{0}, \mathbb{D}_{1}\right)$ be a double category. Then the horizontal nerve of $\mathbb{D}$ is the simplicial object $N_{h} \mathbb{D}$ in Cat defined as

$$
\begin{aligned}
\left(N_{h} \mathbb{D}\right)_{0} & =\mathbb{D}_{0} \\
\left(N_{h} \mathbb{D}\right)_{1} & =\mathbb{D}_{1} \\
\left(N_{h} \mathbb{D}\right)_{n} & =\underbrace{\mathbb{D}_{1 t} \times_{s} \mathbb{D}_{1 t} \times_{s} \cdots_{t} \times_{s} \mathbb{D}_{1}}_{n \text { copies of } \mathbb{D}_{1}} .
\end{aligned}
$$

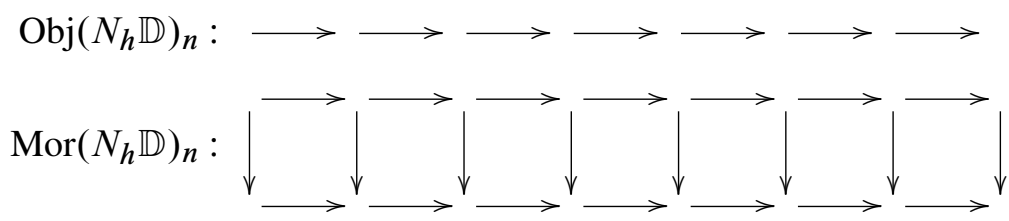

In other words,

$$
\begin{aligned}
\operatorname{Obj}\left(N_{h} \mathbb{D}\right)_{n} & =\operatorname{Cat}([n],(\operatorname{Obj} \mathbb{D}, \operatorname{Hor} \mathbb{D})) \\
\operatorname{Mor}\left(N_{h} \mathbb{D}\right)_{n} & =\operatorname{Cat}([n],(\operatorname{Ver} \mathbb{D}, \operatorname{Sq} \mathbb{D})) .
\end{aligned}
$$

Composition in $\left(N_{h} \mathbb{D}\right)_{n}$ is vertical.

There is of course the analogous notion of vertical nerve of $\mathbb{D}$ denoted $N_{v} \mathbb{D}$. 
Example 5.2 If $\mathbf{C}$ is a category, then the simplicial set $N_{h}(\mathbb{H} \mathbf{C})$ is the usual nerve of C. The horizontal nerve $N_{h}$ is preferred for this reason. The horizontal embedding $\mathbb{H}$, in turn, is preferred because it is the functor

$$
\text { Cat }=\text { Cat }(\text { Set }) \longrightarrow \text { Cat }(\text { Cat })=\text { DblCat }
$$

induced by the embedding Set $\longrightarrow$ Cat . In other words, considering a category $\mathbf{C}$ as a double category with discrete object category and discrete morphism category is the same as identifying it with $\mathbb{H} \mathbf{C}$.

Like the nerve of a category, the horizontal nerve of a double category has a representable definition. Recall that DblCat $\mathbf{v}_{\mathbf{v}}$ denotes the 2-category of small double categories, double functors, and vertical natural transformations.

Proposition 5.3 For every double category $\mathbb{D}$, the simplicial category

$$
[n] \mapsto \operatorname{DblCat}_{\mathbf{v}}(\mathbb{H}[n], \mathbb{D})
$$

is isomorphic to the horizontal nerve $N_{h} \mathbb{D}$. Equivalently, the object simplicial set of the horizontal nerve is

$$
[n] \mapsto \operatorname{DblCat}([0] \otimes[n], \mathbb{D})
$$

and the morphism simplicial set of the horizontal nerve is

$$
[n] \mapsto \operatorname{DblCat}([1] \otimes[n], \mathbb{D}) .
$$

Proof The double categories $\mathbb{H}[n]$ and $[0] \otimes[n]$ are isomorphic, and vertical natural transformations between double functors $\mathbb{H}[n] \longrightarrow \mathbb{D}$ are the same as double functors

$$
[1] \otimes[n]=(\mathbb{H}[1])^{t} \times \mathbb{H}[n] \longrightarrow \mathbb{D}
$$

as pointed out by Grandis-Paré [42].

Proposition 5.3 makes the functoriality of $N_{h}$ immediate. Even more, if we make Cat $^{\Delta^{\mathrm{op}}}$ into a 2-category with 2-cells the modifications, then $N_{h}$ becomes a 2-functor.

Corollary 5.4 The horizontal nerve is a 2-functor

$$
N_{h}: \text { DblCat }_{\mathbf{v}} \longrightarrow \text { Cat }^{\Delta^{\mathrm{op}}} .
$$

In Section 6 we construct the left adjoint to the horizontal nerve explicitly, but for now we observe that a left adjoint exists. Recall the Enriched Lemma from Kan, which follows from Theorem 4.51 of Kelly's monograph [54]. 
Theorem 5.5 (Enriched Lemma from Kan) Let $\mathcal{V}$ be a symmetric monoidal closed category with small homsets. Suppose $\mathbf{A}$ is a small $\mathcal{V}$-category, $\mathbf{B}$ is a cocomplete $\mathcal{V}$-category, and $J: \mathbf{A} \longrightarrow \mathbf{B}$ is a $\mathcal{V}$-functor. Then the enriched left Kan extension of $J$ along the Yoneda embedding exists and is the enriched left adjoint of the singular functor

$$
\begin{gathered}
J_{*}: \mathbf{B} \longrightarrow \mathcal{V}^{\mathbf{A}^{\mathrm{op}}} \\
B \longmapsto \mathbf{B}(J(-), B) .
\end{gathered}
$$

Theorem 5.6 The horizontal nerve $N_{h}$ : DblCat $\longrightarrow$ Cat $^{\Delta^{\mathrm{op}}}$ admits a left 2-adjoint $c_{h}$ called horizontal categorification.

Proof Let $\mathcal{V}$ be Cat, and let $J: \mathbf{A} \longrightarrow \mathbf{B}$ be the Cat-functor

$$
\mathbb{H}: \Delta \longrightarrow \text { DblCat }_{\mathbf{v}} \text {. }
$$

By Proposition 5.3 the horizontal nerve is $J_{*}$.

Since the 2-category DblCat $_{\mathbf{v}}$ is 2-cocomplete by Theorem 4.2, and $\Delta$ is small, we may now apply Theorem 5.5 to obtain the left 2 -adjoint $c_{h}$.

Theorem 5.7 The horizontal nerve $N_{h}$ preserves filtered colimits.

Proof It follows from Theorem 4.7 that the category of horizontal morphisms and squares of a filtered colimit of double categories is the filtered colimit of the categories of horizontal morphisms and squares. Since filtered colimits commute with finite limits, in particular iterated pullbacks, $N_{h}$ preserves filtered colimits.

The horizontal nerve is also well behaved with respect to external products.

Proposition 5.8 Let $\sigma:$ Cat $\longrightarrow$ Cat $^{\Delta^{\text {op }}}$ denote the constant functor. Let

$$
v: \mathbf{S e t}^{\Delta^{\mathrm{op}}} \longrightarrow \mathbf{C a t}^{\Delta^{\mathrm{op}}}
$$

be the inclusion induced by the functor Set $\longrightarrow$ Cat which takes a set to the corresponding discrete category. If $\mathbf{A}$ and $\mathbf{B}$ are categories, then $N_{h}(\mathbf{A} \otimes \mathbf{B})=\sigma \mathbf{A} \times v N \mathbf{B}$. In other words, $N_{h}(\mathbf{A} \otimes \mathbf{B})_{k}=\mathbf{A} \times N \mathbf{B}_{k}$ where we view the set $N \mathbf{B}_{k}$ as a discrete category.

Like the traditional nerve, the horizontal nerve is fully faithful. 
Proposition 5.9 The horizontal nerve $N_{h}$ : DblCat $_{\mathbf{v}} \longrightarrow$ Cat $^{\Delta^{\mathrm{op}}}$ is fully faithful in the 2-categorical sense, that is, the functors

$$
\text { DblCat }_{\mathbf{v}}(\mathbb{D}, \mathbb{E}) \stackrel{\left(N_{h}\right)_{\mathbb{D}, \mathbb{E}}}{\longrightarrow} \mathbf{C a t}^{\Delta^{\mathrm{op}}}\left(N_{h} \mathbb{D}, N_{h} \mathbb{E}\right)
$$

are isomorphisms of categories.

Proof The data of a double functor $F$ and a vertical natural transformation $\sigma$ are encoded entirely in $N_{h} F$ and $N_{h} \sigma$, so $\left(N_{h}\right)_{\mathbb{D}, \mathbb{E}}$ is injective on objects and injective on morphisms.

If $F^{\prime}: N_{h} \mathbb{D} \longrightarrow N_{h} \mathbb{E}$ is a morphism in $\mathbf{C a t}^{\Delta^{\mathrm{op}}}$, then the functors $F_{0}^{\prime}$ and $F_{1}^{\prime}$ give the data for a double functor $F$, and compatibility with face and degeneracy maps guarantees compatibility of $F$ with horizontal composition and units. From $\left(N_{h} F\right)_{0}=$ $F_{0}^{\prime}$ and $\left(N_{h} F\right)_{1}=F_{1}^{\prime}$ it follows that $N_{h} F=F^{\prime}$ using the compatibility with the injective maps $e_{i, i+1}:\{0,1\} \longrightarrow\{0, \ldots, n\}$ defined by

$$
\begin{aligned}
& e_{i, i+1}(0)=i \\
& e_{i, i+1}(1)=i+1
\end{aligned}
$$

for $0 \leq i \leq n-1$. Similarly, if $\sigma^{\prime}$ is a $2-$ cell in $\mathbf{C a t}^{\Delta^{\mathrm{op}}}$, we can construct a vertical natural transformation $\sigma$ from $\sigma_{0}^{\prime}$ and $\sigma_{1}^{\prime}$ such that $N_{h} \sigma=\sigma^{\prime}$. Then $\left(N_{h}\right)_{\mathbb{D}, \mathbb{E}}$ is surjective on objects and surjective on morphisms.

Also like the traditional nerve, the horizontal nerve is 2-coskeletal, which means that the component $N_{h} \mathbb{D} \longrightarrow \operatorname{csk}_{2} \operatorname{tr}_{2} N_{h} \mathbb{D}$ of the unit for the adjunction

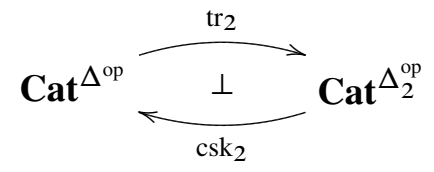

is an isomorphism of simplicial objects in Cat. To prove this, we need a proposition from enriched category theory, the first part of which is Theorem 5.13 of [54].

Proposition 5.10 (Proposition 1.1 of Lack-Paoli [62]) Let $\mathcal{V}$ be a symmetric monoidal closed category which is complete and cocomplete. If

$$
\mathcal{A} \stackrel{I}{\longrightarrow} \mathcal{B} \stackrel{J}{\longrightarrow} \mathcal{C}
$$

are $\mathcal{V}$-functors, and $J$ is fully faithful, then $J$ is dense provided that $J I$ is so, and then the identity $J I=J I$ exhibits $J$ as the left Kan extension of $J I$ along $I$. Furthermore, 
the singular functor $\mathcal{C}(J, 1): \mathcal{C} \longrightarrow\left[\mathcal{B}^{\text {op }}, \mathcal{V}\right]$ can then be obtained by first applying the singular functor

$$
\mathcal{C}(J I, 1): \mathcal{C} \longrightarrow\left[\mathcal{A}^{\text {op }}, \mathcal{V}\right]
$$

and then right Kan extending along $I: \mathcal{A}^{\mathrm{op}} \longrightarrow \mathcal{B}^{\mathrm{op}}$.

Proposition 5.11 The horizontal nerve of a small double category is 2-coskeletal.

Proof For $I$ and $J$ in Proposition 5.10, we take

$$
\Delta_{2} \stackrel{I}{\longrightarrow} \Delta \stackrel{J}{\longrightarrow} \text { DblCat }_{\mathbf{v}}
$$

where $I$ is the inclusion of the full subcategory $\Delta_{2}$ of $\Delta$ on the objects [0], [1] and [2], and $J=\mathbb{H}$ as in the proof of Theorem 5.6. Clearly, $J$ is fully faithful in the 2-categorical sense. We denote by $N_{h}^{2}$ the 2-truncation of the horizontal nerve, which is the singular functor $\operatorname{DblCat}_{\mathbf{v}}(J I, 1)$. By the same argument as in Proposition 5.9, $N_{h}^{2}$ is fully faithful in the 2-categorical sense, which is equivalent to the density of $J I$ according to Theorem 5.1 (ii) of [54]. By Proposition 5.10, the horizontal nerve $N_{h}=\operatorname{DblCat}_{\mathbf{v}}(J, 1)$ is 2-naturally isomorphic to the composite

$$
\text { DblCat }_{\mathbf{v}} \stackrel{N_{h}^{2}}{\longrightarrow} \mathbf{C a t}^{\Delta_{2}^{\mathrm{op}}} \stackrel{\operatorname{Ran}_{I}}{\longrightarrow} \mathbf{C a t}^{\Delta^{\mathrm{op}}} .
$$

Evaluating this 2-natural isomorphism at a double category $\mathbb{D}$, we obtain an isomorphism

$$
N_{h} \mathbb{D} \longrightarrow \operatorname{csk}_{2} \operatorname{tr}_{2} N_{h} \mathbb{D}
$$

between $N_{h} \mathbb{D}$ and a 2-coskeletal simplicial object. From the naturality of the unit, it follows that $N_{h} \mathbb{D}$ is also 2-coskeletal.

Definition 5.12 The classifying space functor $B$ is the composite

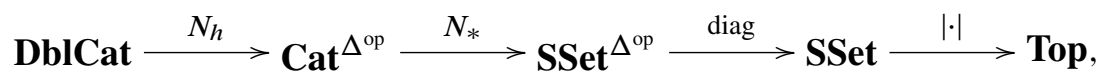

where $N_{*}$ is the levelwise nerve induced by the ordinary nerve $N$, diag is induced by the diagonal functor, and $|\cdot|$ is the geometric realization. The bisimplicial set $N_{*}\left(N_{h}(\mathbb{D})\right)$ is isomorphic to the bisimplicial nerve $N_{d} \mathbb{D}$ of the double category $\mathbb{D}$.

\section{Horizontal categorification}

We now construct a left adjoint $c_{h}$ to the horizontal nerve $N_{h}$ by analogy with the usual nerve $N$. Horizontal categorification $c_{h}$ is appropriately compatible with external products, as we show in Example 6.6 and Proposition 6.11. 
We recall the left adjoint $c$ : SSet $\longrightarrow$ Cat to the nerve functor $N$ constructed by Gabriel-Zisman [37]. For a simplicial set $X$, the category $c X$ is the fundamental category of $X$, or categorification of $X$. It is the free category on the reflexive graph ( $\left.X_{0}, X_{1}\right)$ modulo the smallest congruence such that for every $\tau \in X_{2}$ with edges

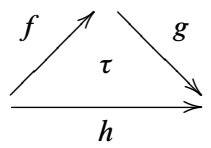

we have $g \circ f \sim h$. The following proof is our guideline for the left adjoint $c_{h}$ to $N_{h}$.

Proposition 6.1 Categorification $c$ is left adjoint to the nerve functor $N$.

Proof We need to construct a natural bijection

$$
\operatorname{Cat}(c X, \mathbf{A}) \cong \operatorname{SSet}(X, N \mathbf{A}) .
$$

Suppose we have a map $G: X \longrightarrow N \mathbf{A}$ of simplicial sets. The $1-$ truncation is a morphism of reflexive graphs, so there is a unique functor $J$ making the upper left triangle commute.

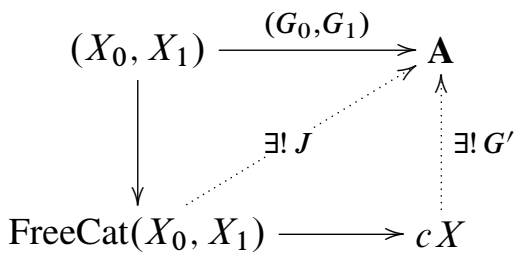

Since $J$ comes from a morphism of simplicial sets, the functor $J$ takes congruent morphisms to equal ones. Therefore there exists a unique functor $G^{\prime}$ making the lower right triangle commute.

For the converse, given a functor $G^{\prime}: c X \longrightarrow \mathbf{A}$, we compose it with the morphism of reflexive graphs

$$
\left(X_{0}, X_{1}\right) \longrightarrow \text { FreeCat }\left(X_{0}, X_{1}\right) \longrightarrow c X
$$

to obtain a morphism of reflexive graphs $\left(G_{0}, G_{1}\right):\left(X_{0}, X_{1}\right) \longrightarrow \mathbf{A}$. We define $G_{2}: X_{2} \longrightarrow(N \mathbf{A})_{2}$ on $\sigma \in X_{2}$ as

$$
\stackrel{G_{1}\left(d_{2} \sigma\right)}{\longrightarrow} \stackrel{G_{1}\left(d_{0} \sigma\right)}{\longrightarrow} .
$$

By definition $G_{2}$ is compatible with $d_{2}$ and $d_{0}$, and the quotient in the definition of $c X$ makes $G_{2}$ compatible with $d_{1}$. This, together with the simplicial identities relating 
$X_{1}$ and $X_{2}$

$$
d_{0} s_{0}=\operatorname{id}_{X_{1}} \quad d_{2} s_{0}=s_{0} d_{1} \quad d_{0} s_{1}=s_{0} d_{0} \quad d_{2} s_{1}=\mathrm{id}_{X_{1}},
$$

implies that $G_{2}$ is also compatible with the degeneracies $s_{0}$ and $s_{1}$. Since $N \mathbf{A}$ is 2-coskeletal this morphism $\left(G_{0}, G_{1}, G_{2}\right)$ of 2-truncated simplicial sets induces a morphism $G: X \longrightarrow N \mathbf{A}$ of simplicial sets:

$$
\operatorname{Set}^{\Delta_{2}^{\mathrm{op}}}\left(\operatorname{tr}_{2} X, \operatorname{tr}_{2} N \mathbf{A}\right) \cong \operatorname{SSet}\left(X, \operatorname{csk}_{2} \operatorname{tr}_{2} N \mathbf{A}\right) \cong \operatorname{SSet}(X, N \mathbf{A}) .
$$

The two procedures $G \mapsto G^{\prime}$ and $G^{\prime} \mapsto G$ are inverse to one another.

Remark 6.2 Any morphism of simplicial sets $G: X \longrightarrow N \mathbf{A}$ is completely determined by its 1 -truncation $\left(G_{0}, G_{1}\right)$ as follows. We let $e_{i, i+1}:\{0,1\} \longrightarrow\{0, \ldots, n\}$ be the injective map defined by

$$
\begin{aligned}
& e_{i, i+1}(0)=i \\
& e_{i, i+1}(1)=i+1
\end{aligned}
$$

for $0 \leq i \leq n-1$, and we let $e_{i}:\{0\} \longrightarrow\{0, \ldots, n\}$ be the injective map defined by

$$
e_{i}(0)=i
$$

for $0 \leq i \leq n$. If $\sigma$ is an $n$-simplex, then $G(\sigma)$ is the string of $n$ morphisms in $\mathbf{A}$

$$
\stackrel{G\left(e_{0,1}^{*}(\sigma)\right)}{\longrightarrow} \stackrel{G\left(e_{1,2}^{*}(\sigma)\right)}{\longrightarrow} \cdots \quad \cdots \stackrel{G\left(e_{n-1, n}^{*}(\sigma)\right)}{\longrightarrow}
$$

where the source and target of $G\left(e_{i, i+1}^{*}(\sigma)\right)$ are $G\left(e_{i}^{*}(\sigma)\right)$ and $G\left(e_{i+1}^{*}(\sigma)\right)$.

We turn next to the left adjoint of the horizontal nerve. We will exhibit two proofs that the horizontal categorification of the product of a category with a simplicial set is an external product of the category with the fundamental category of the simplicial set. This is done in Example 6.6 using the definition of horizontal categorification, while it is done in Proposition 6.11 using weighted colimits.

Definition 6.3 Let $X \in \mathbf{C a t}^{\Delta^{\mathrm{op}}}$. We define a double category $c_{h} X$ called the horizontal categorification or fundamental double category of $X$ as follows. First we define a double derivation scheme $\mathbb{S}$ with vertical 1-category $X_{0}$ and with horizontal 1category the fundamental category of the simplicial set $\operatorname{Obj} X$. The squares of $\mathbb{S}$ are the morphisms of $X_{1}$. We equip the free double category $\mathbb{F}$ on the double derivation scheme $\mathbb{S}$ with the smallest congruence $\sim$ such that:

(1) If $\alpha, \beta \in$ Mor $X_{1}$ are composable in $X_{1}$, then the vertical composite $\left[\begin{array}{l}\alpha \\ \beta\end{array}\right]$ in $\mathbb{F}$ is congruent to the composite of $\beta$ and $\alpha$ in $X_{1}$. 
(2) For all $\tau \in$ Mor $X_{2}$ with boundary

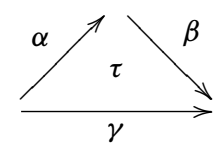

we have

$$
[\alpha \beta] \sim \gamma .
$$

(3) For any vertical morphism $j$, that is, for any $j \in \operatorname{Mor} X_{0}$, the horizontal identity $i_{j}^{h}$ is congruent to the degeneracy of $j$ in Mor $X_{1}$.

(4) For any $f \in \operatorname{Obj} X_{1}$, the vertical identity square $i_{f}^{v}$ on the image of $f$ in the horizontal 1 -category of $\mathbb{S}$ is congruent to the identity on $f$ in the category $X_{1}$.

We define $c_{h} X$ as the quotient of $\mathbb{F}$ by the congruence $\sim$. The horizontal and vertical 1 -categories of $c_{h} X$ are the horizontal and vertical 1 -categories of $\mathbb{S}$.

Remark 6.4 In the definition of horizontal categorification it is not necessary to mod out by additional relations to make the identity squares functorial. If $g \circ f \sim h$ in the horizontal 1-category because of $\tau \in \operatorname{Obj} X_{2}$, then the identity morphism on $\tau$ in the category $X_{2}$ implies we have $i_{h}^{v} \sim\left[i_{f}^{v} i_{g}^{v}\right]$ (the face maps are functors and we have (2) and (4)). For vertically composable morphisms $j$ and $k$, we have

$$
i_{\left[\begin{array}{l}
j \\
k
\end{array}\right]}^{h} \sim\left[\begin{array}{c}
i_{j}^{h} \\
i_{k}^{h}
\end{array}\right]
$$

because degeneracy is a functor and by (1) and (3).

Example 6.5 If $X$ is a simplicial set, then $c_{h} v X=\mathbb{H} c X$. By definition, the horizontal 1 -category is $c X$, and the vertical 1-category is the discrete category $X_{0}$. Since $X_{1}$ is also discrete, there are no nontrivial squares.

Example 6.6 Recall from Proposition 5.8 that $\sigma$ : Cat $\longrightarrow$ Cat $^{\Delta^{\text {op }}}$ denotes the constant functor and $v$ : $\mathbf{S e t}^{\Delta^{\mathrm{op}}} \longrightarrow \mathbf{C a t}^{\Delta^{\mathrm{op}}}$ denotes the inclusion. If $\mathbf{A}$ is a category and $Y$ is a simplicial set, then the horizontal categorification of the simplicial category $\sigma \mathbf{A} \times \nu Y$ is $\mathbf{A} \otimes c Y$. In fact, the horizontal 1-category of the double derivation scheme $\mathbb{S}$ is

$$
c(\operatorname{Obj}(\sigma \mathbf{A} \times v Y))=c(\operatorname{Obj} \mathbf{A} \times Y)=(\operatorname{Obj} \mathbf{A}) \times c Y,
$$

which is the same as the horizontal 1-category of $\mathbf{A} \otimes c Y$. The vertical 1-category of $\mathbb{S}$ is

$$
(\sigma \mathbf{A} \times v Y)_{0}=\mathbf{A} \times Y_{0},
$$


which is the same as the vertical 1-category of $\mathbf{A} \otimes c Y$. The squares of $\mathbb{S}$ are

$$
\operatorname{Mor}(\sigma \mathbf{A} \times v Y)_{1}=(\operatorname{Mor} \mathbf{A}) \times Y_{1} .
$$

The congruence on $\mathbb{F}$ corresponds precisely to the relations in $\mathbf{A} \otimes c Y$ for pairwise compositions of squares and identity squares. We present an alternative conceptual proof of this example in Proposition 6.11.

As we have seen in Theorem 5.6, the horizontal nerve $N_{h}$ admits a left 2-adjoint. We verify that $c_{h}$ as in Definition 6.3 is the underlying 1-functor of the left 2-adjoint.

Proposition 6.7 Horizontal categorification $c_{h}$ is left adjoint to the horizontal nerve $N_{h}$.

Proof We use the notation of Definition 6.3 and construct a natural bijection

$$
\operatorname{DblCat}\left(c_{h} X, \mathbb{D}\right) \cong \operatorname{Cat}^{\Delta^{\mathrm{op}}}\left(X, N_{h} \mathbb{D}\right) .
$$

Suppose $G: X \longrightarrow N_{h} \mathbb{D}$ is a morphism of simplicial objects in Cat. This induces a morphism of double derivation schemes $\mathbb{S} \longrightarrow \mathbb{D}$ and a unique double functor $J$ making the upper left triangle commute:

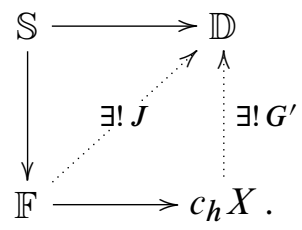

Since $G$ is a morphism of simplicial objects in Cat, $J$ takes congruent squares to equal squares, and there exists a unique double functor $G^{\prime}$ making the lower right triangle commute.

On the other hand, given a double functor $G^{\prime}: c_{h} X \longrightarrow \mathbb{D}$ we compose it with

$$
\left(X_{0}, X_{1}\right) \longrightarrow \mathbb{F} \longrightarrow c_{h} X
$$

to obtain a morphism $\left(G_{0}, G_{1}\right)$ of 1 -truncated simplicial objects in Cat. By the same argument as in the proof of Proposition 6.1, we obtain maps of simplicial sets

$$
\begin{aligned}
G^{\text {Obj: }} \text { Obj } X & \longrightarrow \operatorname{Obj} N_{h} \mathbb{D} \\
G^{\text {Mor }}: \operatorname{Mor} X & \longrightarrow \operatorname{Mor} N_{h} \mathbb{D} .
\end{aligned}
$$

The maps $\left(G_{0}^{\mathrm{Obj}}, G_{0}^{\mathrm{Mor}}\right)$ and $\left(G_{1}^{\mathrm{Obj}}, G_{1}^{\mathrm{Mor}}\right)$ are already known to be functors, and $X$ is a simplicial object in Cat. 
We claim that $\left(G_{2}^{\text {Obj }}, G_{2}^{\text {Mor }}\right)$ is also a functor. If $\sigma, \sigma^{\prime} \in$ Mor $X_{2}$ are composable we denote their composite as

$$
\left[\frac{\sigma}{\sigma^{\prime}}\right]
$$

We similarly denote composites in $\left(N_{h} \mathbb{D}\right)_{2}$. To indicate a composite of a horizontal path of squares in $\mathbb{D}$, we separate the squares by a dot. Then $G_{2}$ preserve compositions

$$
\begin{aligned}
G_{2}\left[\frac{\sigma}{\sigma^{\prime}}\right]=d_{2} G_{2}\left[\frac{\sigma}{\sigma^{\prime}}\right] \cdot d_{0} G_{2}\left[\frac{\sigma}{\sigma^{\prime}}\right] & =G_{1} d_{2}\left[\frac{\sigma}{\sigma^{\prime}}\right] \cdot G_{1} d_{0}\left[\frac{\sigma}{\sigma^{\prime}}\right] \\
=\left[\frac{G_{1} d_{2} \sigma}{G_{1} d_{2} \sigma^{\prime}}\right] \cdot\left[\frac{G_{1} d_{0} \sigma}{G_{1} d_{0} \sigma^{\prime}}\right] & =\left[\frac{G_{1} d_{2} \sigma \cdot G_{1} d_{0} \sigma}{G_{1} d_{2} \sigma^{\prime} \cdot G_{1} d_{0} \sigma^{\prime}}\right] \\
& =\left[\frac{G_{2} \sigma}{G_{2} \sigma^{\prime}}\right],
\end{aligned}
$$

and $G_{2}$ preserves units similarly.

By Proposition 5.11, $N_{h} \mathbb{D}$ is 2-coskeletal, so the 2-truncated morphism $\left(G_{0}, G_{1}, G_{2}\right)$ induces a morphism $X \longrightarrow N_{h} \mathbb{D}$, and by Remark 6.2, this morphism must be $\left(G^{\mathrm{Obj}}, G^{\mathrm{Mor}}\right)$ from above.

The two procedures $G \mapsto G^{\prime}$ and $G^{\prime} \mapsto G$ are inverse to one another.

Proposition 6.8 Consider Cat embedded into Cat $^{\Delta^{\text {op }}}$ as the constant simplicial objects, and consider Cat embedded vertically into DblCat. Then the adjunction $c_{h} \dashv N_{h}$ restricts to the identity adjunction on these full subcategories.

We now move towards a conceptual proof of Example 6.6 in Proposition 6.11.

Remark 6.9 Recall that if $S$ is a set and $A$ is an object of a category, then the copower $S \cdot A$ is the coproduct of $A$ with itself $S$ times. In some categories, the copower has a simple description. For example, if $\mathbf{C}$ is a category, then the copower in Cat is

$$
S \cdot \mathbf{C}=\coprod_{S} \mathbf{C}=S \times \mathbf{C} .
$$

If $X$ is a simplicial set and $Y \in \mathbf{C a t}^{\Delta^{\text {op }}}$, then $X \cdot Y$ is the simplicial object in Cat

$$
[n] \longmapsto X_{n} \cdot Y_{n}=\bigsqcup_{X_{n}} Y_{n}=X_{n} \times Y_{n},
$$

which is the same as $v X \times Y$. 
Lemma 6.10 If $X$ and $Y$ are simplicial objects in Cat, then $X \times Y$ is the weighted colimit $X * G$ of the Cat-functor

$$
\begin{aligned}
& G: \Delta \longrightarrow \mathbf{C a t}^{\Delta^{\text {op }}} \\
& {[n] \longmapsto Y \times v \Delta[n]}
\end{aligned}
$$

with weighting $X: \Delta^{\mathrm{op}} \longrightarrow$ Cat .

Proof Since $($ Cat,$\times)$ is symmetric monoidal closed, it follows from a general fact that $\mathbf{C a t}^{\Delta^{\text {op }}}$ has a tensor product

$$
(Y \otimes Z)_{n}:=Y_{n} \times Z_{n}=(Y \times Z)_{n}
$$

and an internal hom

$$
\begin{aligned}
{[Y, Z]_{n}: } & =\operatorname{Cat}^{\Delta^{\mathrm{op}}}(\Delta[n] \cdot Y, Z) \\
& \cong \mathbf{C a t}^{\Delta^{\mathrm{op}}}(Y \times v \Delta[n], Z)
\end{aligned}
$$

for all $Y, Z \in \mathbf{C a t}^{\Delta^{\mathrm{op}}}$.

For any $Z \in \mathbf{C a t}^{\Delta^{\mathrm{op}}}$,

$$
\mathbf{C a t}^{\Delta^{\mathrm{op}}}(G([n]), Z)
$$

is the $n$-th category of the internal hom $[Y, Z]$. Thus we have a natural isomorphism

$$
\operatorname{Cat}^{\Delta^{\mathrm{op}}}\left(X, \mathbf{C a t}^{\Delta^{\mathrm{op}}}(G(-), Z)\right) \cong \mathbf{C a t}^{\Delta^{\mathrm{op}}}(X \times Y, Z)
$$

and $X \times Y$ satisfies the universal property of the weighted colimit $X * G$.

We finish the conceptual proof of Example 6.6.

Proposition 6.11 If $\mathbf{A}$ is a category and $Y$ is a simplicial set, then the horizontal categorification of the simplicial category $\sigma \mathbf{A} \times v Y$ is $\mathbf{A} \otimes c Y$ where $c Y$ is the traditional categorification of $Y$.

Proof By Lemma 6.10, $\sigma \mathbf{A} \times \nu Y$ is the weighted colimit $\sigma \mathbf{A} * G$ of

$$
\begin{gathered}
G: \Delta \longrightarrow \text { Cat }^{\Delta^{\text {op }}} \\
{[n] \longmapsto v Y \times v \Delta[n]}
\end{gathered}
$$

with weighting $\sigma \mathbf{A}: \Delta^{\mathrm{op}} \longrightarrow$ Cat . 
Let $J: \Delta \longrightarrow$ DblCat be the horizontal embedding. Then by Theorem 4.51 of Kelly's monograph [54], for each $Z \in \mathbf{C a t}^{\Delta^{\mathrm{op}}}, c_{h}(Z) \cong Z * J$. Hence

$$
\begin{aligned}
c_{h}(\sigma \mathbf{A} \times v Y) & =c_{h}(\sigma \mathbf{A} * G) \\
& \cong(\sigma \mathbf{A} * G) * J \\
& \cong \sigma \mathbf{A} *(G * J)
\end{aligned}
$$

by the general Fubini Theorem, which is equation (3.23) in [54]. The functor

$$
G * J: \Delta \longrightarrow \text { DblCat }
$$

in the last line takes $[n]$ to

$$
G([n]) * J \cong c_{h}(G([n]))=c_{h}(v Y \times v \Delta[n]) .
$$

From Example 6.5 and the fact that $c$ preserves finite products, we have

$$
c_{h}(v Y \times v \Delta[n])=\mathbb{H} c(Y \times \Delta[n]) \cong \mathbb{H} c Y \times \mathbb{H}[n] .
$$

We conclude that (5) has the form

$$
c_{h}(\sigma \mathbf{A} \times v Y) \cong \sigma \mathbf{A} *(\mathbb{H} c Y \times \mathbb{H}[-]) .
$$

We claim that the right hand side of (6) is isomorphic to $\mathbb{V A} \times \mathbb{H} c Y$. In fact, Corollary 2.14 and the adjunction $s k_{0} \dashv \operatorname{tr}_{0}$ give, for all $\mathbb{E} \in \mathbf{D b l C a t}_{\mathbf{v}}$

$$
\begin{aligned}
\left.\operatorname{DblCat}_{\mathbf{v}}(\mathbb{V A} \times \mathbb{H} c Y, \mathbb{E})\right) & \cong \operatorname{Cat}\left(\mathbf{A}, \operatorname{DblCat}_{\mathbf{v}}(\mathbb{H} c Y, \mathbb{E})\right) \\
& \cong \operatorname{Cat}\left(\mathbf{A}, \operatorname{tr}_{0} \operatorname{DblCat}_{\mathbf{v}}(\mathbb{H} c Y \times \mathbb{H}[-], \mathbb{E})\right) \\
& \cong \operatorname{Cat}^{\Delta{ }^{\mathrm{op}}}\left(\sigma \mathbf{A}, \operatorname{DblCat}_{\mathbf{v}}(\mathbb{H} c Y \times \mathbb{H}[-], \mathbb{E})\right) .
\end{aligned}
$$

The claim follows now from the definition of weighted colimit. Hence, (6) implies that

$$
c_{h}(\sigma \mathbf{A} \times v Y) \cong \mathbb{V} \mathbf{A} \times \mathbb{H} c Y=\mathbf{A} \otimes c Y .
$$

Remark The vertical categorification of a simplicial object $X$ in Cat is the transpose of $c_{h} X$.

\section{Model structures arising from $\mathrm{Cat}^{\mathrm{u}^{\mathrm{op}}}$}

Now that we have the adjunction $c_{h} \dashv N_{h}$ in place we can use it to transfer model

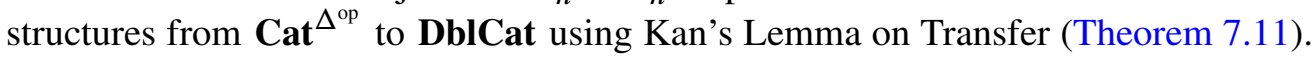
This theorem says that one can lift a model structure across an adjunction under a certain pushout condition and certain smallness conditions, which guarantee functorial factorizations. This is our first method for constructing model structures on DblCat. 
In Section 8 we will adopt the point of view of double categories as internal categories and apply the results of Everaert-Kieboom-Van der Linden [32]. In Section 9 we will consider DblCat as a category of algebras for a 2-monad and use Lack's algebra structure [60].

The category $\mathbf{C a t}^{\Delta^{\mathrm{op}}}$ has four model structures of interest to us. These arise as diagram structures and Reedy structures associated to two cofibrantly generated model structures on Cat: the Thomason structure and the categorical structure. In Sections 7.1-7.4 we review some material for the reader's convenience: model structures on Cat, their associated diagram structures, smallness arguments, and Kan's Lemma on Transfer. After these preliminaries, we turn to our new results. In Sections 7.5 and 7.6 we transfer the diagram structures to DblCat across the horizontal categorification-horizontal nerve adjunction, and show that the transferred structures on DblCat extend the Thomason structure and categorical structure on the vertically embedded subcategory Cat. In the proofs of our transfer results we crucially need to know the behavior of certain pushouts, and these are treated in Theorem 10.6 and Theorem 10.7 of the Appendix. We show in Section 7.7 that the Reedy categorical structure cannot transfer.

Recall the notion of cofibrantly generated model category.

Definition 7.1 A model category $\mathbf{C}$ is cofibrantly generated if there exist sets of morphism $I$ and $J$ in $\mathbf{C}$ such that:

(1) The domains of $I$ are small with respect to $I$-cell as defined in Definition 7.5 and Definition 7.8.

(2) The domains of $J$ are small with respect to $J$-cell.

(3) The class of fibrations is precisely the class of morphisms with the right lifting property with respect $J$.

(4) The class of acyclic fibrations is precisely the class of morphisms with the right lifting property with respect to $I$.

In this case, $I$ is the set of generating cofibrations and $J$ is the set of generating acyclic cofibrations.

\subsection{Model structures on Cat}

In the Thomason structure on Cat in [80] a functor $F$ is a weak equivalence (respectively fibration) if and only if $\mathrm{Ex}^{2} N F$ is a weak equivalence (respectively fibration) of simplicial sets. The functor $\mathrm{Ex}^{2}$ is superfluous for weak equivalences, as Thomason proved that $F$ is a weak equivalence if and only if $N F$ is. The functor Ex: SSet $\longrightarrow$ SSet 
is the right adjoint to barycentric subdivision $\mathrm{Sd}$ : SSet $\longrightarrow$ SSet, which we recall below. The Thomason structure is cofibrantly generated. The generating cofibrations are the inclusions of categorical boundaries

$$
c \mathrm{Sd}^{2} \partial \Delta[m] \longrightarrow c \mathrm{Sd}^{2} \Delta[m],
$$

while the generating acyclic cofibrations are the inclusions of categorical horns

$$
c \mathrm{Sd}^{2} \Lambda^{k}[m] \longrightarrow c \mathrm{Sd}^{2} \Delta[m] .
$$

We now recall the definition of barycentric subdivision $\mathrm{Sd}$. The simplicial sets $\operatorname{Sd} \Delta[m]$ and $\operatorname{Sd} \Lambda^{k}[m]$ are respectively the nerves of the posets of nondegenerate simplices of $\Delta[m]$ and $\Lambda^{k}[m]$. The ordering is the face relation. Thus a $q$-simplex of $\operatorname{Sd} \Delta[m]$ is a tuple $\left(v_{0}, \ldots, v_{q}\right)$ of nondegenerate simplices (faces) of $\Delta[m]$ such that $v_{i}$ is a face of $v_{i+1}$ for all $0 \leq i \leq q-1$. Such a tuple is a $q$-simplex of $\operatorname{Sd} \Lambda^{k}[m]$ if and only if all $v_{0}, \ldots, v_{q}$ are in $\Lambda^{k}[m]$. A $p$-simplex $u$ is a face of a $q$-simplex $v$ in $\operatorname{Sd} \Delta[m]$ if and only if

$$
\left\{u_{0}, \ldots, u_{p}\right\} \subseteq\left\{v_{0}, \ldots, v_{q}\right\} .
$$

A $p$-simplex $u$ of $\operatorname{Sd} \Delta[m]$ is nondegenerate if and only if all $u_{i}$ are distinct.

The barycentric subdivision of a simplicial set $Y$ is by definition

$$
\underset{\Delta[n] \rightarrow Y}{\operatorname{colim}} \operatorname{Sd} \Delta[n]
$$

where the colimit is indexed over the category of simplices of $Y$. It follows from page 311 of [80] that $c \mathrm{Sd}^{2} \Delta[m]$ and $c \operatorname{Sd}^{2} \Lambda^{k}[m]$ are respectively the posets of nondegenerate simplices of $\operatorname{Sd} \Delta[m]$ and $\operatorname{Sd} \Lambda^{k}[m]$ and the generating acyclic cofibration $c \mathrm{Sd}^{2} \Lambda^{k}[m] \longrightarrow c \mathrm{Sd}^{2} \Delta[m]$ is the inclusion of these posets.

The other model structure on Cat is the categorical structure, or natural structure, of Joyal-Tierney [51]. In the categorical structure a functor is a weak equivalence if and only if it is an equivalence of categories. A functor $F: \mathbf{A} \longrightarrow \mathbf{B}$ is a fibration if and only if for each isomorphism $\beta: b \cong F a$ in $\mathbf{B}$ there is an isomorphism $\alpha: a^{\prime} \cong a$ in $\mathbf{A}$ such that $F a^{\prime}=b$ and $F \alpha=\beta$. These fibrations of categories are also called isofibrations. A cofibration is a functor that is injective on objects. The categorical structure on Cat is also cofibrantly generated. There are three generating cofibrations:

$$
\begin{gathered}
\varnothing \hookrightarrow\{1\} \\
\{0,1\} \hookrightarrow\{0 \rightarrow 1\} \\
\{0 \rightrightarrows 1\} \longrightarrow\{0 \rightarrow 1\}
\end{gathered}
$$


and one generating acyclic cofibration:

$$
\{1\} \smile\{0 \cong 1\}=\mathbf{I} .
$$

\subsection{Diagram model structures on $\mathrm{Cat}^{{ }^{\mathrm{\Delta op}}}$}

Given a model category $\mathbf{M}$ and a small category $\mathbf{C}$, one might hope that the category $\mathbf{M}^{\mathbf{C}}$ of functors $\mathbf{C} \longrightarrow \mathbf{M}$ is also a model category with levelwise weak equivalences and levelwise fibrations. By this we mean that a natural transformation is a weak equivalence (respectively fibration) if and only if each of its components is. Unfortunately, this definition does not always give rise to a model structure on $\mathbf{M}^{\mathbf{C}}$. However, if $\mathbf{M}$ is a cofibrantly generated model category, Theorem 7.2 guarantees that this definition does indeed give rise to a model structure on $\mathbf{M}^{\mathbf{C}}$, which is even cofibrantly generated.

Theorem 7.2 (Theorem 11.6.1 in [46]) Let $\mathbf{C}$ be a small category and $\mathbf{M}$ a cofibrantly generated model category with $I$ the set of generating cofibrations and $J$ the set of generating acyclic cofibrations. Then $\mathbf{M}^{\mathbf{C}}$ is a cofibrantly generated model category with levelwise weak equivalences and levelwise fibrations. The generating cofibrations are natural transformations of the form

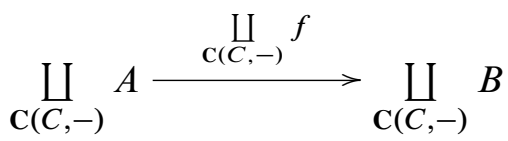

for $f: A \longrightarrow B$ in $I$. The generating acyclic cofibrations are defined similarly with $f$ in $J$. A morphism in $\mathbf{M}^{\mathbf{C}}$ is a cofibration if and only if it is a retract of a transfinite composition of pushouts of generating cofibrations. The components of a cofibration are also cofibrations.

Thus, the category $\mathbf{C a t}^{\Delta^{\text {op }}}$ inherits two model structures from Section 7.1. In the diagram Thomason structure on $\mathbf{C a t}^{\Delta^{\mathrm{op}}}$, a natural transformation $\alpha$ is a weak equivalence (respectively fibration) if and only if $\mathrm{Ex}^{2} N \alpha_{i}$ is a weak equivalence (respectively fibration) of simplicial sets for each $i \geq 0$. In the diagram categorical structure on $\mathbf{C a t}^{\Delta^{\mathrm{op}}}$, a natural transformation $\alpha$ is a weak equivalence (respectively fibration) if and only if $\alpha_{i}$ is an equivalence of categories (respectively isofibration) for all $i \geq 0$.

If $\mathbf{C}$ is a Reedy category, then a model structure on $\mathbf{M}$ also induces a Reedy model structure on $\mathbf{M}^{\mathbf{C}}$ (see for example Hirschhorn [46] or Hovey [47]). The category $\Delta^{\mathrm{op}}$ is a Reedy category, so the Thomason and categorical structures on Cat also give rise to two more model structures on $\mathbf{C a t}^{{ }^{\mathrm{op}}}$. However, we do not study these in more detail because of the following Theorem and also because of Theorem 7.22. 
Theorem 7.3 (Theorem 15.6.4 in [46]) If $\mathbf{C}$ is a Reedy category and $\mathbf{M}$ is a cofibrantly generated model category, then the identity functor of $\mathbf{M}^{\mathbf{C}}$ is a left Quillen equivalence from the cofibrantly generated diagram model structure to the Reedy model structure, and a right Quillen equivalence in the opposite direction.

\subsection{Smallness}

We will need some knowledge about smallness to use Kan's Lemma on Transfer. We recall some of the relevant notions described in Hovey's monograph [47]. Appropriate smallness conditions also allow us to conclude that a transfinite composition of weak equivalences is a weak equivalence.

Definition 7.4 Let $\kappa$ be a cardinal. An ordinal $\lambda$ is $\kappa$-filtered if it is a limit ordinal and, if $A \subseteq \lambda$ and $|A| \leq \kappa$, then $\sup A<\lambda$.

Definition 7.5 Let $\mathbf{C}$ be a category with all small colimits and $\kappa$ a cardinal. An object $A$ of $\mathbf{C}$ is called $\kappa$-small if for all $\kappa$-filtered ordinals $\lambda$ and all colimit-preserving functors $X: \lambda \longrightarrow \mathbf{C}$ the map of sets

$$
\underset{\beta<\lambda}{\operatorname{colim}} \mathbf{C}\left(A, X_{\beta}\right) \longrightarrow \mathbf{C}\left(A, \underset{\beta<\lambda}{\operatorname{colim}} X_{\beta}\right)
$$

is a bijection. An object $A$ is said to be small if it is $\kappa$-small for some cardinal $\kappa$. An object $A$ is said to be finite if it is $\kappa$-small for a finite cardinal $\kappa$, that is, for any limit ordinal $\lambda$ and colimit-preserving functor $X$, the map (7) is a bijection. We say the concepts hold relative to a class of morphisms $\mathbf{D}$ in $\mathbf{C}$ if they hold true for all $X$ with $X_{\beta} \longrightarrow X_{\beta+1}$ in $\mathbf{D}$ for all $\beta+1<\lambda$.

For example, categories are small as follows, and we conclude similarly that double categories are small.

Proposition 7.6 Any category $\mathbf{A}$ is $\kappa$-small where

$$
\kappa=|\operatorname{Obj} \mathbf{A}|+|\operatorname{Mor} \mathbf{A}|+\left|\operatorname{Mor} \mathbf{A}_{s} \times_{t} \operatorname{Mor} \mathbf{A}\right| .
$$

In particular, if Mor $\mathbf{A}$ is a finite set, then $\mathbf{A}$ is finite as an object of Cat.

Proof Let $\mathbf{X}: \lambda \longrightarrow$ Cat be a colimit-preserving functor from a $\kappa$-filtered ordinal $\lambda$. Recall that ordinals are filtered categories and filtered colimits of categories are formed by simply taking the filtered colimits of the object set and the morphism set.

Suppose $F: \mathbf{A} \longrightarrow$ colim $\mathbf{X}$ is a functor. For each $A \in \operatorname{Obj} \mathbf{A}$ and $f \in$ Mor $\mathbf{A}$ there are ordinals $\alpha_{1}(A)$ and $\alpha_{2}(f)$ such that $F(A)$ and $F(f)$ are in the image of $\mathbf{X}_{\alpha_{1}(A)}$ 
and $\mathbf{X}_{\alpha_{2}(A)}$. Let $\beta$ be the supremum of all the $\alpha_{1}(A)$ and $\alpha_{2}(f)$. Then $\beta<\lambda$ and we obtain maps of sets

$$
\begin{aligned}
G^{\text {Obj }}: \text { Obj } \mathbf{A} & \longrightarrow \text { Obj } \mathbf{X}_{\beta} \\
G^{\text {Mor }}: \text { Mor } \mathbf{A} & \longrightarrow \text { Mor } \mathbf{X}_{\beta}
\end{aligned}
$$

which factor the functor $F$. There exists for each $f \in$ Mor $\mathbf{A}$ an index $\gamma(f)$ such that $s(G(f))=G(s(f))$ and $t(G(f))=G(t(f))$ in $\mathbf{X}_{\gamma(f)}$. For each $A \in \operatorname{Obj} \mathbf{A}$ there is an index $\delta(A)$ such that $G\left(1_{A}\right)=1_{G(A)}$ in $\mathbf{X}_{\delta(A)}$. For each $(\ell, k) \in \operatorname{Mor} \mathbf{A}_{s} \times{ }_{t}$ Mor $\mathbf{A}$ there exists an index $\epsilon(\ell, k)$ such that $G(\ell \circ k)=G(\ell) \circ G(k)$ in $\mathbf{X}_{\epsilon(\ell, k)}$. Let $\zeta$ be the supremum of all these indices $\gamma, \delta, \epsilon$. Then $\zeta<\lambda$ and $G$ induces a functor $\mathbf{A} \longrightarrow \mathbf{X}_{\zeta}$ which factors $F$. Hence (7) is onto.

Suppose $M: \mathbf{A} \longrightarrow \mathbf{X}_{\alpha}$ and $N: \mathbf{A} \longrightarrow \mathbf{X}_{\beta}$ are functors that become equal in the colimit. Then for each $A \in \operatorname{Obj} \mathbf{A}$ and each $f \in \operatorname{Mor} \mathbf{A}$ there are indices $\gamma(A)$ and $\delta(f)$ such that $M(A)=N(A)$ and $M(f)=N(f)$ in $\mathbf{X}_{\gamma(A)}$ and $\mathbf{X}_{\delta(f)}$ respectively. Let $\zeta<\lambda$ be the supremum of all these indices $\gamma(A)$ and $\delta(f)$. Then $M$ and $N$ become equal at the stage $\zeta$ and the map (7) is injective.

Proposition 7.7 Let $\mathbb{D}$ be a double category and $s^{h}, s^{v}, t^{h}, t^{v}$ the horizontal and vertical source and target maps. Then $\mathbb{D}$ is $\kappa$-small where

$$
\begin{aligned}
\kappa= & |\operatorname{Obj} \mathbb{D}|+\mid \text { Hor } \mathbb{D}|+| \text { Hor } \mathbb{D} s_{s^{h} \times}{ }_{t^{h}} \text { Hor } \mathbb{D} \mid \\
& +|\operatorname{Ver} \mathbb{D}|+\mid \operatorname{Ver} \mathbb{D} s_{s^{v} \times t^{v}} \text { Ver } \mathbb{D} \mid \\
& +|\operatorname{Sq} \mathbb{D}|+\left|\operatorname{Sq} \mathbb{D} s^{v} \times{ }_{t} \operatorname{Sq} \mathbb{D}\right| \\
& +\left|\operatorname{Sq} \mathbb{D}{ }_{s^{h} \times}{ }_{t^{h}} \mathrm{Sq} \mathbb{D}\right| .
\end{aligned}
$$

In particular, if $\mathrm{Sq} \mathbb{D}$ is a finite set, then $\mathbb{D}$ is finite as an object of DblCat.

Proof We first obtain a map of the underlying quadruple of sets, and then we go out far enough to make it into a double functor by considering the various compositions and identities as in Proposition 7.6.

Note that this proposition easily generalizes to $n$-fold categories.

One useful application of finiteness is to transfinite compositions of weak equivalences.

Definition 7.8 If $\mathbf{C}$ is a category with all small colimits, $\lambda$ is an ordinal, $\mathbf{D}$ is a class of morphisms in $\mathbf{C}$, and $X: \lambda \longrightarrow \mathbf{C}$ is a colimit preserving functor such that $X_{\beta} \longrightarrow X_{\beta+1}$ is in $\mathbf{D}$ for all $\beta+1<\lambda$, then the morphism

$$
X_{0} \longrightarrow \operatorname{colim} X
$$


is called a transfinite composition of morphisms in $\mathbf{D}$. If $I$ is a class of morphisms in $\mathbf{C}$, then a transfinite composition of pushouts of elements of $I$ is called a relative $I$-cell complex. The class of relative $I$-cell complexes is denoted $I-$ cell.

Proposition 7.9 (Corollary 7.4.2 in [47]) Suppose C is a cofibrantly generated model category in which the domains and codomains of the generating cofibrations and generating acyclic cofibrations are finite. Then every transfinite composition of weak equivalences is a weak equivalence.

Example 7.10 In both the Thomason structure and the categorical structure on Cat, every transfinite composition of weak equivalences is a weak equivalence, as the domains and codomains of the generating cofibrations and generating acyclic cofibrations only have finitely many morphisms. Since weak equivalences and colimits in $\mathbf{C a t}^{\Delta^{\mathrm{op}}}$ are levelwise, every transfinite composition of weak equivalences in the diagram structures is also a weak equivalence.

\subsection{Kan's Lemma on Transfer}

Our first main tool for constructing model structures on DblCat is Kan's Lemma on Transfer. The form we will use is Corollary 7.12.

Theorem 7.11 (Kan's Lemma on Transfer, Theorem 11.3.2 in [46]) Let $\mathbf{C}$ be a cofibrantly generated model category with generating cofibrations $I$ and generating acyclic cofibrations $J$. Suppose $\mathbf{D}$ is complete and cocomplete, and that

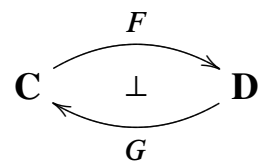

is an adjunction. Assume the following.

(1) For every $i \in I$, dom $F i$ is small with respect to $F I-c e l l$. For every $j \in J$, $\operatorname{dom} F j$ is small with respect to $F J$-cell.

(2) The functor $G$ maps every relative $F J$-complex to a weak equivalence in $\mathbf{C}$.

Then there exists a cofibrantly generated model structure on $\mathbf{D}$ with generating cofibrations $F I$ and generating acyclic cofibrations $F J$. Further, $f$ is a weak equivalence in $\mathbf{D}$ if and only $G(f)$ is a weak equivalence in $\mathbf{C}$, and $f$ is a fibration in $\mathbf{D}$ if and only $G(f)$ is a fibration in $\mathbf{C}$. 
Along the lines of Proposition 3.4.1 of Worytkiewicz-Hess-Parent-Tonks [83], we have the following corollary.

Corollary 7.12 Let $\mathbf{C}$ be a cofibrantly generated model category with generating cofibrations $I$ and generating acyclic cofibrations $J$. Suppose $\mathbf{D}$ is complete and cocomplete, and that $F \dashv G$ is an adjunction as in (8). Assume the following.

(1) For every $i \in I$ and $j \in J$, the objects $\operatorname{dom} F i$ and $\operatorname{dom} F j$ are small with respect to the entire category $\mathbf{D}$.

(2) For any ordinal $\lambda$ and any colimit preserving functor $X: \lambda \longrightarrow \mathbf{C}$ such that $X_{\beta} \longrightarrow X_{\beta+1}$ is a weak equivalence, the transfinite composition

$$
X_{0} \longrightarrow \operatorname{colim} X
$$

is a weak equivalence.

(3) $G$ preserves filtered colimits.

(4) If $j^{\prime}$ is a pushout of $F(j)$ in $\mathbf{D}$ for $j \in J$, then $G\left(j^{\prime}\right)$ is a weak equivalence in C.

Then there exists a cofibrantly generated model structure on $\mathbf{D}$ with generating cofibrations $F I$ and generating acyclic cofibrations $F J$. Further, $f$ is a weak equivalence in $\mathbf{D}$ if and only $G(f)$ is a weak equivalence in $\mathbf{C}$, and $f$ is a fibration in $\mathbf{D}$ if and only $G(f)$ is a fibration in $\mathbf{C}$.

Proof Clearly, (1) of Theorem 7.11 follows from the hypotheses. To see (2), we recall that a relative $F J$-complex is a transfinite composition of pushouts of morphisms $F j$ where $j \in J$. If a relative $F J$-complex $f$ is a transfinite composition of $Y: \lambda \longrightarrow \mathbf{D}$, then $G f$ is the transfinite composition of $X=G \circ Y$. Since $G f$ is a transfinite composition of weak equivalences, $G f$ is also a weak equivalence. Hence $G$ takes relative $F J$-complexes to weak equivalences in $\mathbf{C}$.

\subsection{Transfer of the diagram Thomason structure on $\mathrm{Cat}^{\mathrm{\Delta}^{\mathrm{op}}}$}

With these preliminaries and our free constructions on double categories, we can transfer the diagram Thomason structure to DblCat. Recall the diagram Thomason structure on $\mathbf{C a t}^{\Delta{ }^{\text {op }}}$ from Section 7.2.

Theorem 7.13 There is a cofibrantly generated model structure on DblCat such that a double functor $K$ is a weak equivalence (respectively fibration) if and only if $N_{h} K$ is levelwise a weak equivalence (respectively fibration) in the Thomason structure on Cat. 
Proof We apply Corollary 7.12 to the adjunction $F=c_{h} \dashv N_{h}=G$. First we point out that

$$
\begin{aligned}
c_{h}\left(\coprod_{\Delta^{\mathrm{op}}([n],-)} c \operatorname{Sd}^{2} \Lambda^{k}[m]\right) & =c_{h}\left(c \operatorname{Sd}^{2} \Lambda^{k}[m] \times \Delta[n]\right) \\
& =\left(c \operatorname{Sd}^{2} \Lambda^{k}[m]\right) \otimes c \Delta[n] \\
& =\left(c \operatorname{Sd}^{2} \Lambda^{k}[m]\right) \otimes[n]
\end{aligned}
$$

by Example 6.6 or Proposition 6.11 (for simplicity we suppress $\sigma$ and $v$ ). Similarly,

$$
c_{h}\left(\coprod_{\Delta^{\mathrm{op}}([n],-)} c \mathrm{Sd}^{2} \Delta[m]\right)=\left(c \mathrm{Sd}^{2} \Delta[m]\right) \otimes[n]
$$

and the horizontal categorifications of the generating acyclic cofibrations $j=f \times 1_{\Delta[n]}$ in Theorem 7.2 are the inclusions $f \otimes 1_{[n]}$ for the inclusions

$$
f: c \mathrm{Sd}^{2} \Lambda^{k}[m] \longrightarrow c \mathrm{Sd}^{2} \Delta[m]
$$

and $m \geq 1, n \geq 0$.

(1) The double categories $\left(c \operatorname{Sd}^{2} \partial \Delta[m]\right) \otimes[n]$ and $\left(c \operatorname{Sd}^{2} \Lambda^{k}[m]\right) \otimes[n]$ have a finite number of squares, hence they are finite by Proposition 7.7.

(2) A transfinite composition of weak equivalences in $\mathbf{C a t}^{\Delta^{\mathrm{op}}}$ is a weak equivalence by Example 7.10.

(3) The horizontal nerve $N_{h}$ preserves filtered colimits by Theorem 5.7.

(4) Consider the pushout in DblCat:

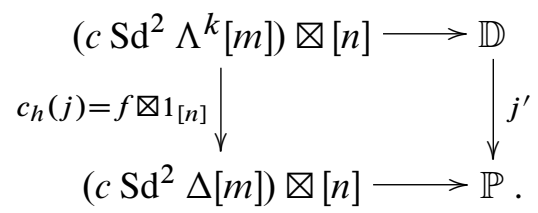

Then by Proposition $5.8 N_{h} c_{h}(j)$ is the acyclic cofibration $j$, and by Theorem 10.7 the diagram

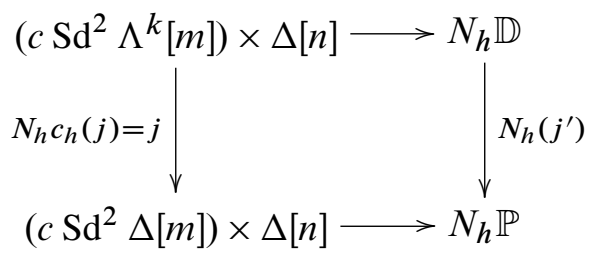

is a pushout in $\mathbf{C a t}^{\Delta^{\mathrm{op}}}$. Hence $N_{h}\left(j^{\prime}\right)$ is an acyclic cofibration, and in particular a weak equivalence in $\mathbf{C a t}^{\Delta^{\mathrm{op}}}$. 
We may compare the transferred diagram Thomason model structure on DblCat to the Thomason model structure on Cat as follows.

Proposition 7.14 The functor $(\mathrm{V}-)_{0}$ : DblCat $\longrightarrow$ Cat maps the weak equivalences and fibrations of the transferred diagram Thomason model structure on DblCat to weak equivalences and fibrations in the Thomason model structure on Cat. In particular, $(\mathbf{V}-)_{0}$ is a right Quillen functor.

Proof As functors, $(\mathbf{V}-)_{0}$ and $\left(N_{h^{-}}\right)_{0}$ are the same.

Corollary 7.15 The adjunction

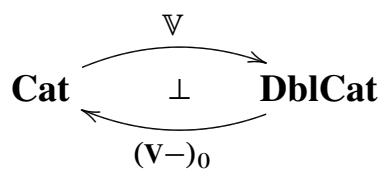

is a Quillen adjunction.

Proof An adjunction is a Quillen adjunction if and only if the right adjoint is a right Quillen functor, so the Corollary follows immediately from Proposition 7.14.

Proposition 7.16 The functor $\mathbb{V}$ : Cat $\longrightarrow$ DblCat preserves and reflects weak equivalences, fibrations, and cofibrations. In other words, a functor $F$ is a weak equivalence (respectively fibration, respectively cofibration) in the Thomason model structure on Cat if and only if $\mathbb{V} F$ is a weak equivalence (respectively fibration, respectively cofibration) in the transferred diagram Thomason model structure on DblCat. As a consequence, the transferred diagram Thomason model structure on DblCat extends the Thomason model structure on Cat as a vertically embedded subcategory.

Proof For a functor $F$, the morphism $N_{h} \mathbb{V} F$ of simplicial objects in Cat is $F$ in every degree. Thus $\mathbb{V}$ preserves and reflects weak equivalences and fibrations.

By Corollary 7.15, $\mathbb{V}$ preserves cofibrations. It also reflects cofibrations as follows. If $F$ is a functor such that $\mathbb{V} F$ is a cofibration, and $G$ is an acyclic fibration in Cat, then any diagram in DblCat

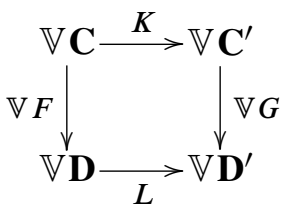

admits a lift, as $\mathbb{V} G$ is an acyclic fibration by the above. Since $\mathbb{V}$ is fully faithful, this lift gives us a lift in Cat. Hence $F$ is a cofibration. 


\subsection{Transfer of the diagram categorical structure on $\mathrm{Cat}^{\mathrm{\Delta}^{\mathrm{op}}}$}

Our preparations allow us to also quickly transfer the diagram categorical structure. Recall the diagram categorical structure on $\mathbf{C a t}^{\Delta^{\mathrm{op}}}$ from Section 7.2. In Section 8.2 we will show that the vertical analogue of this transferred model structure on DblCat coincides with the model structure induced by the simplicially surjective topology $\tau$ on Cat using the methods of Everaert-Kieboom-Van der Linden [32]. An important reason for interest in the equality of these two structures lies in the fact that the second construction yields an explicit form for the cofibrant replacement, which is not at all transparent using only the transferred structure.

Theorem 7.17 There is a cofibrantly generated model structure on DblCat such that a double functor $K$ is a weak equivalence (respectively fibration) if and only if $N_{h} K$ is levelwise a weak equivalence (respectively fibration) in the categorical structure on Cat.

Proof We apply Corollary 7.12 to the adjunction $F=c_{h} \dashv N_{h}=G$. All generating acyclic cofibrations $j$ in Theorem 7.2 for the categorical diagram structure on $\mathbf{C a t}^{\Delta^{\text {op }}}$ are natural transformations of the form

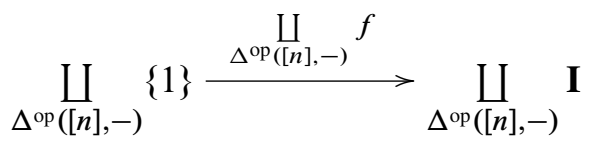

where $f$ is the inclusion $\{1\} \longrightarrow \mathbf{I}$ and $[n]$ is an object of $\Delta^{\mathrm{op}}$. These generating acyclic cofibrations have horizontal categorification

$$
\{1\} \otimes[n] \longrightarrow \mathbf{I} \otimes[n]
$$

by Example 6.6 or Proposition 6.11 (for simplicity we suppress $\sigma$ and $v$ ).

(1) The double categories $\varnothing \otimes[n],\{0,1\} \otimes[n],\{0 \rightrightarrows 1\} \otimes[n]$, and $\{1\} \otimes[n]$ have a finite number of squares, hence they are finite by Proposition 7.7.

(2) A transfinite composition of weak equivalences in $\mathbf{C a t}^{\Delta^{\mathrm{op}}}$ is a weak equivalence by Example 7.10.

(3) The horizontal nerve $N_{h}$ preserves filtered colimits by Theorem 5.7.

(4) Consider the pushout in DblCat:

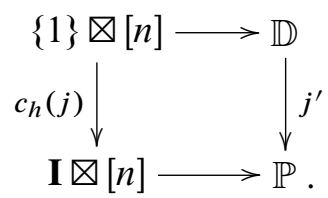


Then by Proposition $5.8 N_{h} c_{h}(j)$ is the acyclic cofibration $j$, and by Theorem 10.7 the diagram

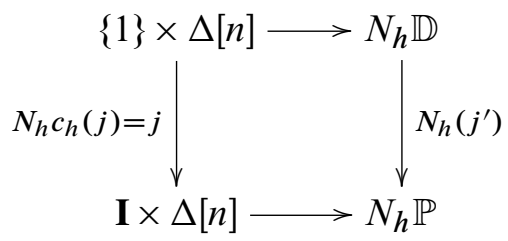

is a pushout in $\mathbf{C a t}^{\Delta^{\text {op }}}$. Hence $N_{h}\left(j^{\prime}\right)$ is an acyclic cofibration, and in particular a weak equivalence in $\mathbf{C a t}^{\Delta^{\mathrm{op}}}$.

We may compare the transferred diagram categorical model structure on DblCat to the categorical model structure on Cat as follows.

Proposition 7.18 The functor $(\mathbf{V}-)_{0}$ : DblCat $\longrightarrow$ Cat maps the weak equivalences and fibrations of the transferred diagram categorical model structure on DblCat to weak equivalences and fibrations in the categorical model structure on Cat. In particular, $(\mathbf{V}-)_{0}$ is a right Quillen functor.

Proof As functors, $(\mathbf{V}-)_{0}$ and $\left(N_{h^{-}}\right)_{0}$ are the same.

Corollary 7.19 The adjunction

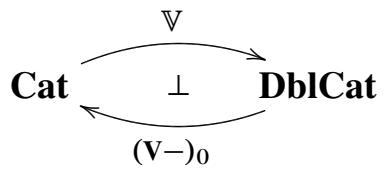

is a Quillen adjunction.

Proof This follows immediately from Proposition 7.18.

Proposition 7.20 The functor $\mathbb{V}$ : Cat $\longrightarrow$ DblCat preserves and reflects weak equivalences, fibrations, and cofibrations. In other words, a functor $F$ is a weak equivalence (respectively fibration, respectively cofibration) in the categorical model structure on Cat if and only if $\mathbb{V} F$ is a weak equivalence (respectively fibration, respectively cofibration) in the transferred diagram categorical model structure on DblCat. As a consequence, the transferred diagram categorical model structure on DblCat extends the categorical model structure on Cat as a vertically embedded subcategory.

Proof The proof is completely analogous to the proof of Proposition 7.16. 


\subsection{No transfer of the Reedy categorical structure on $\mathrm{Cat}^{\mathrm{\Delta}^{\mathrm{op}}}$}

In this section we consider the category $\mathbf{C a t}^{\Delta^{\text {op }}}$ of simplicial objects in Cat equipped with the Reedy model structure associated with the categorical model structure on Cat. The weak equivalences in this Reedy model structure are the levelwise equivalences of categories and the fibrations are the Reedy fibrations. (For further details, see Hirschhorn [46].) In this section we show that it is impossible to transfer this model structure to DblCat via the adjunction $c_{h} \dashv N_{h}$, where $N_{h}$ is the horizontal nerve and $c_{h}$ is the horizontal categorification. We will need the following theorem.

Theorem 7.21 (Theorem 1 of Joyal-Street [50]) For a given functor $G$ : $\mathbf{B} \longrightarrow \mathbf{C}$, the canonical comparison functor from the pullback of $F$ along $G$ to the pseudo pullback of $F$ along $G$ is an equivalence of categories for all functors $F: \mathbf{A} \longrightarrow \mathbf{C}$ if and only if $G$ is an isofibration.

Now we turn to the objective of this subsection.

Theorem 7.22 There does not exist a model structure on DblCat such that a double functor $K$ is a weak equivalence (respectively fibration) if and only if $N_{h} K$ is a weak equivalence (respectively fibration) in the Reedy model structure on $\mathbf{C a t}^{{ }^{\mathrm{op}}}$ associated to the categorical structure on Cat.

Proof Suppose that such a transferred model structure on DblCat does exist. Then $\left(c_{h}, N_{h}\right)$ is a Quillen pair. Let $\mathbb{D}$ be a double category and consider a Reedy cofibrant fibrant replacement $r: N_{h} \mathbb{D} \longrightarrow V_{\bullet}$ in $\mathbf{C a t}^{\Delta^{\text {op }}}$, that is, $V_{\bullet}$ is a Reedy fibrant object and $r$ is an acyclic cofibration in the Reedy structure. Our strategy is to prove that the existence of such a transferred model structure implies a false statement, namely that (14) is an equivalence of categories for every double category $\mathbb{D}$. We then exhibit a double category for which (14) is not an equivalence.

Since $V_{\bullet}$ is Reedy fibrant, the map $\left(d_{0}, d_{1}\right)$ is an isofibration in Cat, which implies that the composite functors $d_{i}$

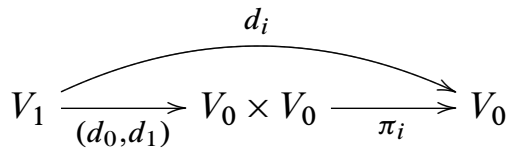

are themselves isofibrations (the two projections $\pi_{i}: V_{0} \times V_{0} \longrightarrow V_{0}$ are clearly isofibrations). By Theorem 7.21 this implies that the canonical functor

$$
V_{1} \times V_{0} V_{1} \longrightarrow V_{1} \stackrel{\text { ps }}{\times} V_{0} V_{1}
$$


from the pullback to the pseudo pullback is an equivalence of categories.

Next we similarly show that (12) is an equivalence of categories. By Definition 6.3 $\left(c_{h} V\right)_{0}=V_{0}$, the set of objects of $V_{1}$ maps to the set of horizontal morphisms of $c_{h} V$, and the set of morphisms of $V_{1}$ maps to the set of squares of $c_{h} V$. We claim that the functor

$$
\left(c_{h} V\right)_{1} \stackrel{\left(d_{0}, d_{1}\right)}{\longrightarrow}\left(c_{h} V\right)_{0} \times\left(c_{h} V\right)_{0}
$$

is also an isofibration. First note that $V_{1} \longrightarrow V_{0} \times V_{0}$ is an isofibration precisely when any diagram in $c_{h} V$ of the special form

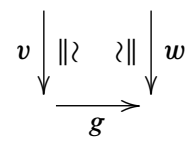

with $v, w$ isomorphisms in $V_{0}$ and $g$ an object of $V_{1}$, can be filled with an isomorphism $\alpha$ in $V_{1}$ (a "generating vertically invertible square" in $c_{h} V$ ):

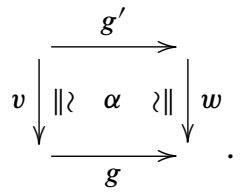

Now consider a general such diagram in $c_{h}(V)$. This has the form

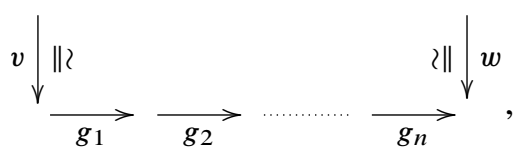

where the bottom edge is an equivalence class of a path of composable horizontal morphisms by Definition 6.3. We next insert vertical identity morphisms and fill in the individual squares to obtain the following allowable compatible arrangement

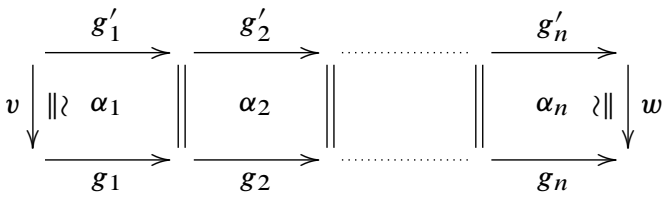

(this is possible because $V_{1} \longrightarrow V_{0} \times V_{0}$ is an isofibration). The equivalence class in $c_{h}(V)$ of this allowable compatible arrangement gives the required filling for (11). 
So (10) is indeed an isofibration. Reasoning as for (9), this implies that the following functor is an equivalence of categories.

$$
\left(c_{h} V\right)_{1} \times\left(c_{h} V\right)_{0}\left(c_{h} V\right)_{1} \longrightarrow\left(c_{h} V\right)_{1} \stackrel{\mathrm{ps}}{\times}\left(c_{h} V\right)_{0}\left(c_{h} V\right)_{1}
$$

We claim that the unit component $\eta_{V}$ is a weak equivalence. First we observe that every component of the counit $\varepsilon: c_{h} N_{h} \Longrightarrow \mathrm{Id}_{\text {DblCat }}$ is an isomorphism of double categories, since the nerve functor $N_{h}$ is fully faithful. Also, one of the triangle identities states that $N_{h} \varepsilon \mathbb{D} \cdot \eta_{N_{h} \mathbb{D}}=$ id, so $\eta_{N_{h} \mathbb{D}}$ is an isomorphism. The naturality of the unit $\eta$ therefore gives us a commutative diagram

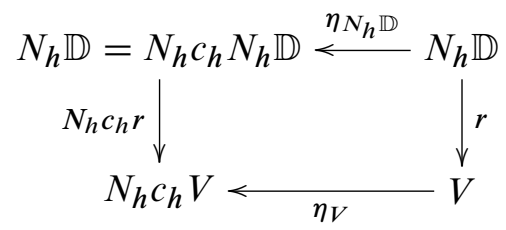

in which the morphism $\eta_{N_{h} \mathbb{D}}$ is a levelwise equivalence (since it is an isomorphism), and the morphism $r$ is a levelwise equivalence (by hypothesis). The morphism $N_{h} c_{h} r$ is one as well, since $c_{h} r$ is a weak equivalence $\left(c_{h} r\right.$ is an acyclic cofibration since $c_{h}$ was assumed to be a left Quillen functor and $r$ is an acyclic cofibration). By the 2-out-of-3 property, it follows that $\eta_{V}$ is a levelwise equivalence of categories, as claimed.

Consider the following commutative diagram in Cat.

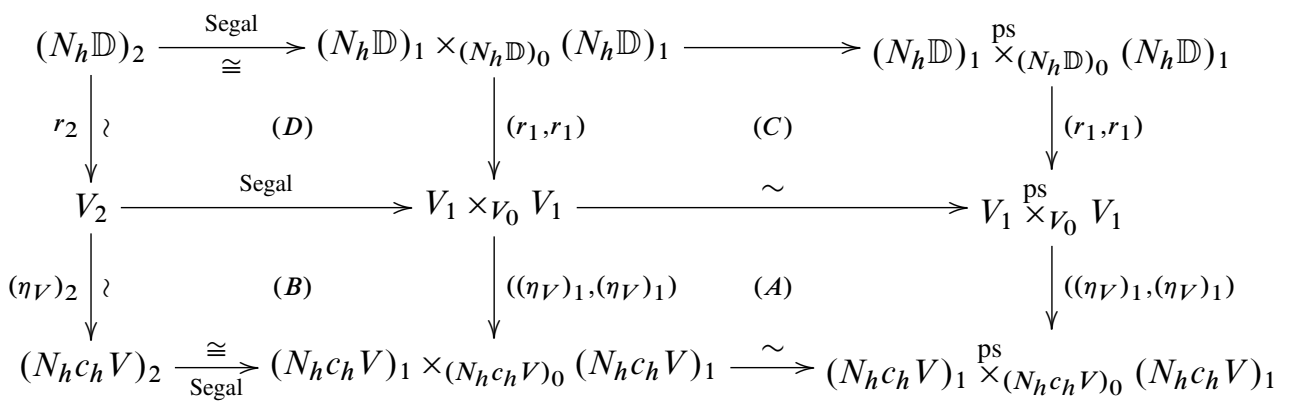

Note that $(B)$ and $(D)$ commute by the definition of Segal maps, while the commutativity of $(A)$ and $(C)$ follows from the universal property of the pseudo pullbacks. The vertical functors $r_{2}$ and $\left(\eta_{V}\right)_{2}$ are equivalences of categories, since $r$ and $\eta_{V}$ are weak equivalences from above. The bottom edge of $(C)$ is an equivalence, since it is (9). The bottom edge of $(A)$ is an equivalence as it is (12) (recall that $\left(N_{h} \mathbb{E}\right)_{0}=\mathbb{E}_{0}$ and $\left(N_{h} \mathbb{E}\right)_{1}=\mathbb{E}_{1}$ for any double category $\left.\mathbb{E}\right)$. 
We claim that the top edge of $(C)$ is an equivalence of categories. Since $r_{0}$ and $r_{1}$ are equivalences, the right vertical functor

$$
\left(r_{1}, r_{1}\right):\left(N_{h} \mathbb{D}\right)_{1} \stackrel{\mathrm{ps}}{\times}\left(N_{h} \mathbb{D}\right)_{0}\left(N_{h} \mathbb{D}\right)_{1} \longrightarrow V_{1} \stackrel{\mathrm{ps}}{\times} V_{0} V_{1}
$$

is an equivalence. Moreover, since $\eta_{V}$ is a levelwise equivalence, the 2-out-of-3 property and the commutativity of $(A)$ imply that the functor

$$
V_{1} \times_{V_{0}} V_{1} \longrightarrow\left(N_{h} c_{h} V\right)_{1} \times\left(N_{h} c_{h} V\right)_{0}\left(N_{h} c_{h} V\right)_{1}
$$

is an equivalence. Also, the commutativity of $(B)$ and the 2-out-of-3 property imply that $V_{2} \longrightarrow V_{1} \times V_{0} V_{1}$ is an equivalence. The commutativity of $(D)$ then implies that

$$
\left(N_{h} \mathbb{D}\right)_{1} \times\left(N_{h} \mathbb{D}\right)_{0}\left(N_{h} \mathbb{D}\right)_{1} \longrightarrow V_{1} \times_{V_{0}} V_{1}
$$

is an equivalence. Finally, the commutativity of $(C)$ implies that the canonical map

$$
\left(N_{h} \mathbb{D}\right)_{1} \times\left(N_{h} \mathbb{D}\right)_{0}\left(N_{h} \mathbb{D}\right)_{1} \longrightarrow\left(N_{h} \mathbb{D}\right)_{1} \stackrel{\text { ps }}{\times}\left(N_{h} \mathbb{D}\right)_{0}\left(N_{h} \mathbb{D}\right)_{1}
$$

is an equivalence of categories, as claimed.

The map (13) is nothing but

$$
\mathbb{D}_{1} \times \mathbb{D}_{0} \mathbb{D}_{1} \longrightarrow \mathbb{D}_{1} \stackrel{\text { ps }}{\times} \mathbb{D}_{0} \mathbb{D}_{1}
$$

The objects of $\mathbb{D}_{1} \stackrel{\mathrm{ps}}{\times \mathbb{D}_{0}} \mathbb{D}_{1}$ are diagrams of the form

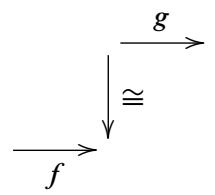

and morphisms of $\mathbb{D}_{1} \stackrel{\mathrm{ps}}{\times} \mathbb{D}_{0} \mathbb{D}_{1}$ are diagrams of the form

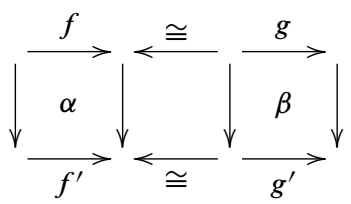


where the middle square is a commutative square of vertical morphisms. The objects and morphisms of $\mathbb{D}_{1} \times_{\mathbb{D}_{0}} \mathbb{D}_{1}$ are those of (15) and (16) where the isomorphisms are identities. The canonical functor (14) is given by this inclusion.

We now exhibit a double category $\mathbb{D}$ where the canonical functor (14) is not an equivalence, which then implies that our original assumption on the existence of a transferred Reedy structure on DblCat is false. Let $\mathbb{D}$ be the double category with four distinct objects $A, B, C, D$ and only the following nontrivial morphisms.

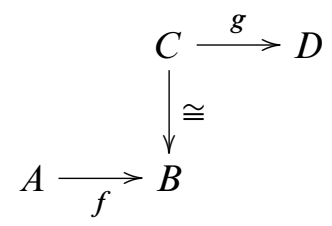

There are no nontrivial squares. Suppose that the canonical functor (14) is essentially surjective. Then there exist objects $X, Y, Z$ and morphisms as in the following diagram

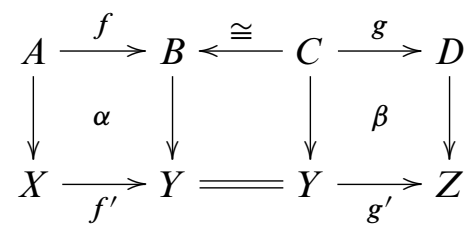

with $\alpha$ and $\beta$ vertically invertible squares. However, since all squares in $\mathbb{D}$ are trivial, we conclude that $B=Y=C$, a contradiction. Hence, the canonical functor (14) is not essentially surjective and is not an equivalence.

We conclude that it is impossible to transfer the categorical Reedy model structure on $\mathrm{Cat}^{\Delta^{\mathrm{op}}}$ to DblCat.

\section{Model structures arising from Grothendieck topologies}

Until now we have considered model structures transferred from $\mathbf{C a t}^{\Delta^{\mathrm{op}}}$. But one can also view double categories as internal categories, and for these a homotopy theory has already been developed. Model structures on internal categories in a category $\mathbf{C}$ satisfying certain hypotheses have been studied by Everaert-Kieboom-Van der Linden [32]. As they point out, there are various notions of internal equivalence of internal categories. The notions full and faithful representably make sense for internal functors as in Definition 8.8, but notions of essential surjectivity depend on a class of morphisms $\mathcal{E}$ in $\mathbf{C}$. If this class of morphisms is the class $\mathcal{E}_{\mathcal{T}}$ of $\mathcal{T}$-epimorphisms for a Grothendieck topology $\mathcal{T}$ on $\mathbf{C}$, then the internal equivalences are the weak equivalences for a model 
structure on $\operatorname{Cat}(\mathbf{C})$ in good cases. The classes we $(\mathcal{T}), \operatorname{fib}(\mathcal{T})$, and $\operatorname{cof}(\mathcal{T})$ are defined in [32] so that the following theorem holds. We will recall the classes we $(\mathcal{T}), \operatorname{fib}(\mathcal{T})$, and $\operatorname{cof}(\mathcal{T})$ below.

Theorem 8.1 (5.5 of [32]) Let $\mathbf{C}$ be a finitely complete category such that Cat(C) is finitely complete and finitely cocomplete and $\mathcal{T}$ is a Grothendieck topology on $\mathbf{C}$. If the class we $(\mathcal{T})$ of $\mathcal{T}$-equivalences has the 2-out-of-3 property and $\mathbf{C}$ has enough $\mathcal{T}$-projectives, then

$$
(\operatorname{Cat}(\mathbf{C}), \operatorname{we}(\mathcal{T}), \operatorname{fib}(\mathcal{T}), \operatorname{cof}(\mathcal{T}))
$$

is a model category. 4

We apply this theorem to $\mathbf{C}=\mathbf{C a t}$ for various Grothendieck topologies in this section. In Section 8.2 we show that the model structure associated to the simplicially surjective topology $\tau$ is the same as the vertically transferred diagram categorical structure. This second construction using [32] is advantageous, as it gives us more information about the model structures, such as simple descriptions of cofibrations and cofibrant replacements. We will show in Section 9 that the model structure associated to the categorically surjective basis $\tau^{\prime}$ in Section 8.3 turns out to be the same as the model structure on DblCat viewed as a category of algebras over a 2 -monad. We also show that the trivial topology induces the trivial model structure associated to the 2-category DblCat $_{h}$.

\subsection{Homotopy theory of internal categories according to Everaert- Kieboom-Van der Linden [32]}

First we recall the notions and results of Everaert-Kieboom-Van der Linden [32], specialized to the case of internal categories in $\mathbf{C}=\mathbf{C a t}$.

Definition 8.2 Let iso: $\operatorname{Cat}($ Cat $) \longrightarrow \operatorname{Grpd}($ Cat $)$ be the right adjoint to the inclusion $\operatorname{Grpd}($ Cat $) \longrightarrow \operatorname{Cat}(\mathbf{C a t})$. For $\mathbb{B} \in \operatorname{Cat}($ Cat $)$, this means that iso $(\mathbb{B})_{1}$ has objects the invertible horizontal morphisms of $\mathbb{B}$ and morphisms the horizontally invertible squares. The category iso $(\mathbb{B})_{1}$ is a subcategory of $\mathbb{B}_{1}$. Composition is the vertical composition of squares.

\footnotetext{
${ }^{4}$ As Tim Van der Linden pointed out to us, the factorizations in this model structure are functorial if there exists a functor $P: \mathbf{C} \longrightarrow \mathbf{C}$ and a natural transformation $\eta: P \Longrightarrow \operatorname{Id}_{\mathbf{C}}$ such that $P(C)$ is $\mathcal{T}$-projective and $\eta_{C}$ is a $\mathcal{T}$-epimorphism for all objects $C$ of $\mathbf{C}$. This is the case with the $\tau$-topology, the $\tau^{\prime}$-topology, and the trivial topology we consider in this paper.
} 
Definition 8.3 If $F: \mathbb{A} \longrightarrow \mathbb{B}$ is a double functor, then the mapping path object is the category $\left(\mathbb{P}_{F}\right)_{0}$ defined as the pullback below:

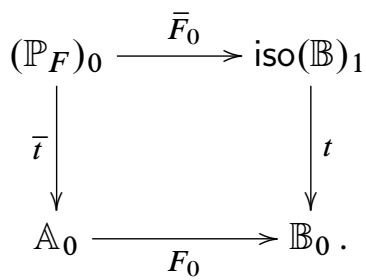

The functor $t$ : iso $(\mathbb{B})_{1} \longrightarrow \mathbb{B}_{0}$ is the target for horizontal composition, exactly as in Definition 2.1. The objects of $\left(\mathbb{P}_{F}\right)_{0}$ are

$$
\left(a, f: b \stackrel{\cong}{\longrightarrow} F_{0} a\right)
$$

for $a$ an object of $\mathbb{A}$ and $f$ a horizontal isomorphism of $\mathbb{B}$. The morphisms are pairs

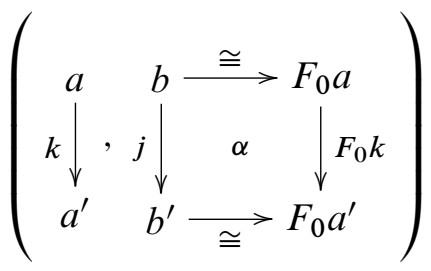

where $k$ is a vertical morphism in $\mathbb{A}$ and $\alpha$ is a horizontally invertible square in $\mathbb{B}$. Composition in $\left(\mathbb{P}_{F}\right)_{0}$ comes from the vertical composition in $\mathbb{A}$ and $\mathbb{B}$.

Definition 8.4 Let $\mathcal{T}$ be a topology on Cat. We denote by $Y_{\mathcal{T}}$ the composition of the Yoneda embedding $Y$ with the sheafification functor.

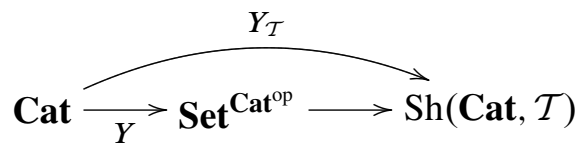

A functor $p: \mathbf{E} \longrightarrow \mathbf{B}$ is a $\mathcal{T}$-epimorphism if $Y_{\mathcal{T}}(p)$ is an epimorphism. In this case, we often simply say that $p$ is $\mathcal{T}$-epi. We denote the class of $\mathcal{T}$-epimorphisms by $\mathcal{E}_{\mathcal{T}}$.

To show that a functor is $\mathcal{T}$-epi, we will use the following characterization of $\mathcal{T}-$ epimorphisms.

Proposition 8.5 (Corollary III.7.5 and III.7.6 of Mac Lane-Moerdijk [65], Proposition 2.12 in Everaert-Kieboom-Van der Linden [32]) Let $\mathcal{T}$ be a topology on a small category. A morphism $p: E \longrightarrow B$ is $\mathcal{T}$-epi if and only if for every morphism 
$g: X \longrightarrow B$ there exists a covering sieve $\left\{f_{i}: U_{i} \longrightarrow X\right\}_{i}$ and a family of morphisms $\left\{u_{i}: U_{i} \longrightarrow E\right\}_{i}$ such that for every $i \in I$ the following diagram commutes.

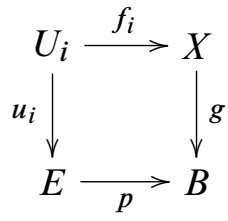

Remark 8.6 Suppose $K$ is a basis for the topology $\mathcal{T}$ in Proposition 8.5 and such $g$ and $p$ are given. Then there exists a covering sieve $\left\{f_{i}: U_{i} \longrightarrow X\right\}_{i}$ in $\mathcal{T}$ and a family of morphisms $\left\{u_{i}: U_{i} \longrightarrow E\right\}_{i}$ making (18) commute if and only if there exists a covering family $\left\{g_{j}: V_{j} \longrightarrow X\right\}_{j}$ in $K$ and a family of morphisms $\left\{v_{j}: V_{j} \longrightarrow E\right\}_{j}$ making (18) commute. Thus, in Proposition 8.5 one could equivalently replace the phrase "covering sieve" by the phrase "covering family in a given basis".

Proof A sieve $S$ is a covering sieve in the topology $\mathcal{T}$ generated by the basis $K$ if and only if it contains a covering family $R$ from the basis $K$. Suppose such a covering sieve $\left\{f_{i}\right\}_{i}$ with morphisms $\left\{u_{i}\right\}_{i}$ is given. Then this covering sieve contains a covering family in $L$ for which (18) commutes. Conversely, given such a covering family $\left\{g_{j}\right\}_{j}$ with morphisms $\left\{v_{j}\right\}_{j}$, we may take the sieve

$$
\left\{g_{j} \circ w \mid w \text { a morphism such that } g_{j} \circ w \text { exists }\right\}_{j}
$$

generated by the family $\left\{v_{j}\right\}_{j}$. Then the family $\left\{v_{j} \circ w\right\}$ makes (18) commute.

Example 8.7 Suppose $\mathcal{T}$ is a topology on a small category. If a morphism $p$ admits a right inverse $q$, then $p$ is a $\mathcal{T}$-epimorphism. To see this using Proposition 8.5, take any covering family $\left\{f_{i}\right\}$ of $X$ and the morphisms $u_{i}=q g f_{i}$.

Definition 8.8 Let $s$ : iso $(\mathbb{B})_{1} \longrightarrow \mathbb{B}_{0}$ be the source map for horizontal composition, as in Definition 2.1. A double functor $F: \mathbb{A} \longrightarrow \mathbb{B}$ is essentially $\mathcal{T}$-surjective if the 
functor

and

$$
\left(\mathbb{P}_{F}\right)_{0} \stackrel{\bar{F}_{0}}{\longrightarrow} \text { iso }(\mathbb{B})_{1} \stackrel{s}{\longrightarrow} \mathbb{B}_{0}
$$

given by

$$
s \circ \bar{F}_{0}\left(a, b \stackrel{\cong}{\longrightarrow} F_{0} a\right)=b,
$$$$
s \circ \bar{F}_{0}\left(\begin{array}{cccc}
a & b & \cong & F_{0} a \\
k \downarrow & j & \alpha & \mid F_{0} k \\
a^{\prime} & b^{\prime} & & \stackrel{1}{\longrightarrow} F_{0} a^{\prime}
\end{array}\right)=j_{\downarrow}
$$

is a $\mathcal{T}$-epimorphism. If $F$ is additionally fully faithful in the sense of Bunge-Paré [19], that is, if

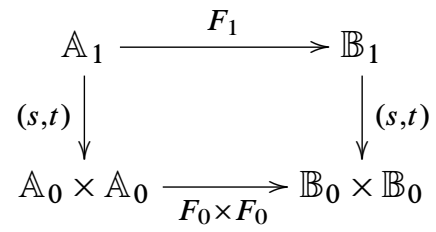

is a pullback square in Cat, then $F$ is called a $\mathcal{T}$-equivalence. We denote the class of $\mathcal{T}$-equivalences by we $(\mathcal{T})$. Note that a double functor $F$ is fully faithful if and only if its restrictions to the two functors

$$
\begin{aligned}
(\operatorname{Obj} \mathbb{A}, \operatorname{Hor} \mathbb{A}) & \longrightarrow(\operatorname{Obj} \mathbb{B}, \operatorname{Hor} \mathbb{B}) \\
(\operatorname{Ver} \mathbb{A}, \text { Sq } \mathbb{A}) & \longrightarrow(\operatorname{Ver} \mathbb{B}, \operatorname{Sq} \mathbb{B})
\end{aligned}
$$

are both fully faithful.

Remark 8.9 If $\mathbf{A}$ and $\mathbf{B}$ are 1-categories, then a functor $F: \mathbf{A} \longrightarrow \mathbf{B}$ is essentially surjective in the usual sense if and only if $s \circ \bar{F}_{0}$ is surjective. The functor $F$ is fully faithful in the sense of Definition 8.8 if and only if it is fully faithful in the usual sense. The notions of essential surjectivity and fully faithfulness can be found in any standard reference on category theory, such as pages 19 and 115 of Borceux [10] or pages 14, 15 and 93 of Mac Lane [64].

Definition 8.10 Let $\mathcal{E}$ be a class of functors. We say that a category $\mathbf{P}$ is projective with respect to the class $\mathcal{E}$ if for every functor $G: \mathbf{Q} \longrightarrow \mathbf{R}$ in $\mathcal{E}$ and every functor 
$H: \mathbf{P} \longrightarrow \mathbf{R}$ there exists a functor $F: \mathbf{P} \longrightarrow \mathbf{Q}$ such that $G F=H:$

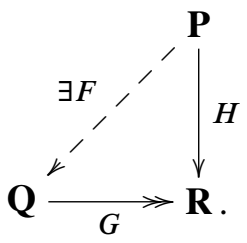

The double arrow head signifies that $G$ is in $\mathcal{E}$. If $\mathcal{E}$ is the class $\mathcal{E}_{\mathcal{T}}$ of $\mathcal{T}$-epi functors, then a projective category $\mathbf{P}$ is called $\mathcal{T}$-projective. ${ }^{5}$

Definition 8.11 We say that there are enough $\mathcal{T}$-projectives in Cat if for every category $\mathbf{C}$ there exists a $\mathcal{T}$-projective category $\mathbf{P}$ and a $\mathcal{T}$-epi functor $\mathbf{P} \longrightarrow \mathbf{C}$.

Definition 8.12 A double functor $F: \mathbb{E} \longrightarrow \mathbb{B}$ is a $\mathcal{T}$-fibration if the induced morphism $\left(r_{F}\right)_{0}$ in the diagram below is a $\mathcal{T}$-epimorphism:

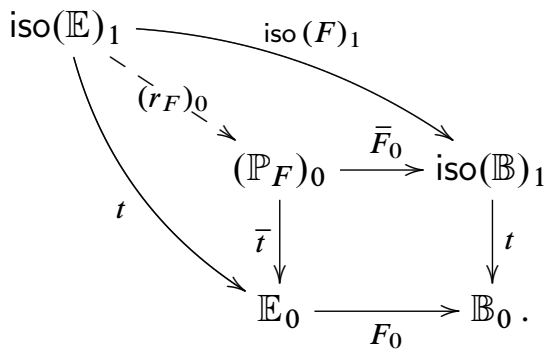

On objects, $\left(r_{F}\right)_{0}$ is

$$
\left(f^{\prime}: b^{\prime} \stackrel{\cong}{\longrightarrow}\right) \mapsto\left(e, F f^{\prime}: F b^{\prime} \cong F e\right) .
$$

Remark 8.13 If $\mathbf{E}$ and $\mathbf{B}$ are 1-categories, then $\left(r_{F}\right)_{0}$ is surjective if and only if $F$ is an isofibration. Recall from Section 7.1 that a functor $F: \mathbf{E} \longrightarrow \mathbf{B}$ is said to be an isofibration if for any object $e$ of $\mathbf{E}$ and any isomorphism $f: b \cong F e$ in $\mathbf{B}$, there exists a lift to an isomorphism $f^{\prime}: b^{\prime} \cong e$ in $\mathbf{E}$.

Example 8.14 (Example 5.2 of [32]) If 1 denotes the terminal double category, then the unique double functor $\mathbb{D} \longrightarrow \mathbf{1}$ is a $\mathcal{T}$-fibration for every topology $\mathcal{T}$ on Cat. Hence, in the model structure of Theorem 8.1, every object is fibrant.

\footnotetext{
${ }^{5}$ This was also called $\mathcal{E}_{\mathcal{T}}$-projective in [32].
} 
Proof In diagram (19), the functor $\bar{t}$ is the identity, so that $\left(r_{F}\right)_{0}$ is simply $t$. A right inverse assigns horizontal identities to objects, and horizontal identity squares to vertical morphisms. By Example 8.7, the functor $\left(r_{F}\right)_{0}$ is a $\mathcal{T}$-epimorphism.

Proposition 8.15 (Proposition 5.6 of [32]) A double functor $F: \mathbb{E} \longrightarrow \mathbb{B}$ is an acyclic $\mathcal{T}$-fibration if and only if it is fully faithful and $F_{0}$ is a $\mathcal{T}$-epi functor.

Definition 8.16 A double functor is a $\mathcal{T}$-cofibration if it has the left lifting property with respect to all acyclic $\mathcal{T}$-fibrations.

Proposition 8.17 (Proposition 5.9 of [32]) A double functor $J: \mathbb{A} \longrightarrow \mathbb{X}$ is a $\mathcal{T}$ cofibration if and only if $J_{0}$ has the left lifting property with respect to all $\mathcal{T}$-epi functors.

Corollary 8.18 A double category $\mathbb{X}$ is cofibrant in the $\mathcal{T}$-model structure if and only if $\mathbb{X}_{0}$ is $\mathcal{T}$-projective.

Proof By Proposition 8.17, $\mathbb{X}$ is cofibrant if and only if for any $\mathcal{T}$-epi functor $G$ and any functor $H$, a lift $\ell$

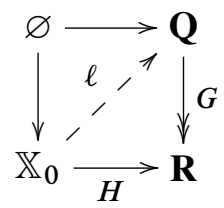

exists, or equivalently, $\mathbb{X}_{0}$ is $\mathcal{T}$-projective.

Remark 8.19 These results allow us to construct a cofibrant replacement $\mathbb{E}$ for a double category $\mathbb{B}$ in the $\mathcal{T}$-model structure. Let $\mathbb{E}_{0}$ be a $\mathcal{T}$-projective category and $K_{0}: \mathbb{E}_{0} \longrightarrow \mathbb{B}_{0}$ a $\mathcal{T}$-epimorphism (we will explicitly give $\mathbb{E}_{0}$ and $K_{0}$ in the $\tau$-and $\tau^{\prime}$-structures in Remark 8.31 and Remark 8.48). Let $\mathbb{E}_{1}$ be the following pullback in Cat:

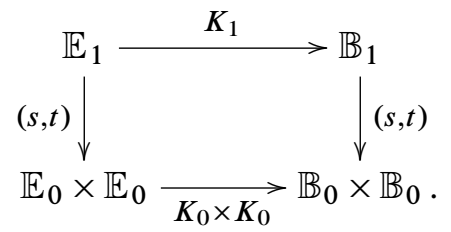

Then the double graph $\mathbb{E}$ carries a unique double category structure such that $K=$ $\left(K_{0}, K_{1}\right)$ is a double functor by Lemma 5.14 of [32]. Since $K$ is fully faithful and $K_{0}$ is $\mathcal{T}$-epi, $K: \mathbb{E} \longrightarrow \mathbb{B}$ is an acyclic $\mathcal{T}$-fibration by Proposition 8.15. By Corollary $8.18, \mathbb{E}$ is a cofibrant double category, and hence a cofibrant replacement for $\mathbb{B}$ in the $\mathcal{T}$-model structure. 
Remark 8.20 We conclude from Proposition 8.5 that if $\mathcal{T}^{\prime} \subseteq \mathcal{T}$ are Grothendieck topologies, then every $\mathcal{T}^{\prime}$-epimorphism is a $\mathcal{T}$-epimorphism. Thus we conclude from Definitions 8.8, 8.12 and 8.16 that if $\mathcal{T}^{\prime} \subseteq \mathcal{T}$ then we $\left(\mathcal{T}^{\prime}\right) \subseteq \operatorname{we}(\mathcal{T})$, fib $\left(\mathcal{T}^{\prime}\right) \subseteq \operatorname{fib}(\mathcal{T})$, and $\operatorname{cof}\left(\mathcal{T}^{\prime}\right) \supseteq \operatorname{cof}(\mathcal{T}) .^{6}$

\subsection{Model structure from the simplicially surjective basis}

For a category $\mathbf{C}$, we write $\mathbf{C}_{k}$ for the $k$-th set of the nerve $N \mathbf{C}$. Similarly for a functor $F$ we write $(N F)_{k}=F_{k}$. We say that a functor $F$ is simplicially surjective if $F_{k}$ is surjective for all $k \geq 0$. We prove that the associated topology on Cat induces a model structure on DblCat which coincides with the vertical analogue of the transferred diagram categorical structure of Section 7.6. This second construction gives additional information about (the vertical analogue of) the transferred diagram categorical structure, including an explicit form for the cofibrant replacement functor.

Lemma 8.21 For a category $\mathbf{C}$ define

$$
K(\mathbf{C}):=\{\{F: \mathbf{D} \longrightarrow \mathbf{C}\} \mid F \text { a simplicially surjective functor }\} .
$$

Then $K$ is a basis for a Grothendieck topology $\tau$ on Cat.

\section{Proof}

(1) If $F$ is an isomorphism, then $N F$ is an isomorphism and each $F_{k}$ is bijective.

(2) If $\{F\} \in K(\mathbf{C})$ and $G: \mathbf{C}^{\prime} \longrightarrow \mathbf{C}$ is any functor, consider the pullback $\pi_{2}: \mathbf{D} \times \mathbf{C} \mathbf{C}^{\prime} \longrightarrow \mathbf{C}^{\prime}$ in Cat of $F$ along $G$. Since the nerve functor preserves limits, $N \pi_{2}$ is the pullback of $N F$ along $N G$. Then $N \pi_{2}$ is simplicially surjective, since limits of simplicial sets are formed pointwise.

(3) If $G \circ F$ exists and $F_{k}$ and $G_{k}$ are surjective for all $k \geq 0$, then clearly $G_{k} \circ F_{k}$ is surjective for all $k \geq 0$, and $\{G \circ F\}$ is a covering family.

Lemma 8.22 A functor $p$ : $\mathbf{E} \longrightarrow \mathbf{B}$ is $\tau$-epi for the Grothendieck topology $\tau$ if and only if $p$ is simplicially surjective.

Proof If $p$ is $\tau$-epi, then take $g=1_{\mathbf{B}}$ in Proposition 8.5 with Remark 8.6 to obtain $p u=f$ for some covering family $\{f\}$ in $K$. Then $f_{k}$ is surjective for all $k \geq 0$. Hence $p$ is simplicially surjective.

If $p$ is simplicially surjective, then $\{p\}$ is a covering family in $K$, and so is the pullback $\pi_{2}$ of $p$ along $g$. Applying Proposition 8.5 with Remark 8.6 again, we see that $p$ is $\tau$-epi.

\footnotetext{
${ }^{6}$ We thank Joachim Kock for posing this question.
} 
Recall that the objects of the $k$-th category $\left(\left(\mathbb{A}_{0}\right)_{k},\left(\mathbb{A}_{1}\right)_{k}\right)=\left(N_{v} \mathbb{A}\right)_{k}$ of the vertical nerve are composable strings of $k$ vertical morphisms, and the morphisms are vertically composable strings of $k$ squares. The composition is horizontal composition of vertical strings of squares. Fully faithful double functors and $\tau$-equivalences have a useful characterization in terms of the vertical nerve.

Proposition 8.23 A double functor $F: \mathbb{A} \longrightarrow \mathbb{B}$ is fully faithful if and only if for every $k \geq 0$ the functor

$$
\left(\left(F_{0}\right)_{k},\left(F_{1}\right)_{k}\right):\left(\left(\mathbb{A}_{0}\right)_{k},\left(\mathbb{A}_{1}\right)_{k}\right) \longrightarrow\left(\left(\mathbb{B}_{0}\right)_{k},\left(\mathbb{B}_{1}\right)_{k}\right)
$$

is fully faithful.

Proof Since the nerve functor preserves pullbacks, and pullbacks of simplicial sets are formed pointwise, it follows from Definition 8.8 that $F$ is fully faithful if and only if each $\left(\left(F_{0}\right)_{k},\left(F_{1}\right)_{k}\right)$ is fully faithful.

Proposition 8.24 A double functor $F: \mathbb{A} \longrightarrow \mathbb{B}$ is a $\tau$-equivalence if and only if for every $k \geq 0$ the functor

$$
\left(\left(F_{0}\right)_{k},\left(F_{1}\right)_{k}\right):\left(\left(\mathbb{A}_{0}\right)_{k},\left(\mathbb{A}_{1}\right)_{k}\right) \longrightarrow\left(\left(\mathbb{B}_{0}\right)_{k},\left(\mathbb{B}_{1}\right)_{k}\right)
$$

is an equivalence of categories.

Proof The double functor $F$ is essentially $\tau$-surjective if and only if $s \circ \bar{F}_{0}$ is $\tau$-epi. But this occurs if and only if $\left(s \circ \bar{F}_{0}\right)_{k}$ is surjective for each $k$, which is equivalent to the essential surjectivity of $\left(\left(F_{0}\right)_{k},\left(F_{1}\right)_{k}\right)$ by Remark 8.9. Fully faithfulness follows from Proposition 8.23.

Corollary 8.25 The class we $(\tau)$ of $\tau$-equivalences has the 2-out-of-3 property.

Proposition 8.26 Cat has enough $\tau$-projectives.

Proof We first construct a $\tau$-projective category $\mathbf{P}$ from a category $\mathbf{C}$. Let

$$
\mathbf{P}:=\coprod_{n \geq 0} \mathbf{C}_{n} \cdot[n]=\left(\coprod_{c \in \mathbf{C}_{0}}[0]\right) \coprod\left(\coprod_{n \geq 1} \coprod_{\left(f_{1}, \ldots, f_{n}\right) \in \mathbf{C}_{n}}[n]\right)
$$

where $[n]=\{0,1, \ldots, n\}$ is the $(n+1)$-element ordinal viewed as a category and $\mathbf{C}_{n} \cdot[n]$ denotes the copower of the category $[n]$ with the set $\mathbf{C}_{n}$, as recalled in Remark 6.9. 
Suppose we have functors

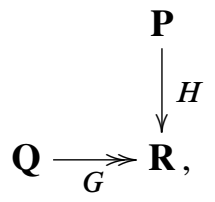

and $G$ is $\tau$-epi. We denote $H$ on the $\left(f_{1}, \ldots, f_{n}\right)$-summand of $\mathbf{P}$ by

$$
H_{\left(f_{1}, \ldots, f_{n}\right)}:[n] \longrightarrow \mathbf{R}
$$

and by $H_{c}$ on the $c$-summand. If $H_{\left(f_{1}, \ldots, f_{n}\right)}(j-1 \leq j)=r_{j}$ for $1 \leq j \leq n$, then there exists $\left(q_{1}, \ldots, q_{n}\right) \in \mathbf{Q}_{n}$ such that $G_{n}\left(q_{1}, \ldots, q_{n}\right)=\left(r_{1}, \ldots, r_{n}\right)$ since $G$ is $\tau$-epi. We define a functor

$$
\begin{gathered}
F_{\left(f_{1}, \ldots, f_{n}\right)}:[n] \longrightarrow \mathbf{Q} \\
F_{\left(f_{1}, \ldots, f_{n}\right)}(j-1 \leq j):=q_{j}
\end{gathered}
$$

for $1 \leq j \leq n$. Similarly, for $c \in \mathbf{C}_{0}$, there exists $d \in \mathbf{Q}_{0}$ such that $G_{0}(d)=H_{c}(0)$. We define $F_{c}(0)=d$. Putting these $F$ 's together, we obtain a functor $F: \mathbf{P} \longrightarrow \mathbf{Q}$ such that $G F=H$, and we conclude that $\mathbf{P}$ is $\tau$-projective.

Next we construct a $\tau$-epi functor $L: \mathbf{P} \longrightarrow \mathbf{C}$. On the $\left(f_{1}, \ldots, f_{n}\right)$-summand of $\mathbf{P}$ define $L$ as

$$
L_{\left(f_{1}, \ldots, f_{n}\right)}(j-1 \leq j):=f_{j}
$$

for $1 \leq j \leq n$. Similarly on the $c$ summand, $L_{c}(0):=c$. We claim that for each $k \geq 0$, $L_{k}: \mathbf{P}_{k} \longrightarrow \mathbf{C}_{k}$ is surjective. Note that

$$
\mathbf{P}_{k}=\left(\coprod_{c \in \mathbf{C}_{0}}[0]_{k}\right) \coprod\left(\coprod_{n \geq 1} \coprod_{\left(f_{1}, \ldots, f_{n}\right) \in \mathbf{C}_{n}}[n]_{k}\right) \text {. }
$$

If $k \geq 1$, and $\left(f_{1}, \ldots, f_{k}\right) \in \mathbf{C}_{k}$, then $L_{k}$ maps $(0 \leq 1,1 \leq 2, \ldots, k-1 \leq k)$ in the $\left(f_{1}, \ldots, f_{k}\right)$-component of $\mathbf{P}_{k}$ to $\left(f_{1}, \ldots, f_{k}\right)$. Similarly if $c \in \mathbf{C}_{0}$, then $L_{0}$ maps 0 in the $c$-component of $\mathbf{P}_{0}$ to $c$. Hence $L_{k}$ is surjective for all $k \geq 0, L$ is $\tau$-epi, and Cat has enough $\tau$-projectives.

Theorem 8.27 The simplicially surjective topology $\tau$ on Cat determines a model structure

$$
(\text { Cat }(\text { Cat }), \operatorname{we}(\tau), \operatorname{fib}(\tau), \operatorname{cof}(\tau)) \text {. }
$$

Proof The category Cat(Cat) is complete and cocomplete by Theorem 4.1. The class of $\tau$-equivalences has the 2-out-of-3 property by Corollary 8.25 and Cat has enough $\tau$-projectives by Proposition 8.26, so we can apply Theorem 8.1. 
We now give a more explicit description of the fibrations, acyclic fibrations, fibrant objects, and cofibrant replacement.

Proposition 8.28 Let $F: \mathbb{E} \longrightarrow \mathbb{B}$ be a double functor.

(1) $F$ is a $\tau$-fibration if and only if for each $k \geq 0$ the functor

$$
\left(\left(F_{0}\right)_{k},\left(F_{1}\right)_{k}\right):\left(\left(\mathbb{E}_{0}\right)_{k},\left(\mathbb{E}_{1}\right)_{k}\right) \longrightarrow\left(\left(\mathbb{B}_{0}\right)_{k},\left(\mathbb{B}_{1}\right)_{k}\right)
$$

is an isofibration.

(2) $F$ is an acyclic $\tau$-fibration if and only if the functor $\left(\left(F_{0}\right)_{k},\left(F_{1}\right)_{k}\right)$ is fully faithful and surjective on objects for each $k \geq 0$.

\section{Proof}

(1) Applying the nerve to Diagram (19), we see that $F$ is a $\tau$-fibration if and only if $\left(r_{F}\right)_{0 k}$ is surjective for all $k \geq 0$. By Remark 8.13, this is the case if and only if for each $k \geq 0$ the functor $\left(\left(F_{0}\right)_{k},\left(F_{1}\right)_{k}\right)$ is an isofibration. Here (iso $\left.(\mathbb{B})_{1}\right)_{k}=$ iso $\left(\left(\mathbb{B}_{0}\right)_{k},\left(\mathbb{B}_{1}\right)_{k}\right)$ is the category with objects composable strings of $k$ vertical morphisms and with morphisms vertical strings of vertically composable squares that are each horizontally invertible.

(2) From Proposition 8.23, $F$ is fully faithful if and only if each $\left(\left(F_{0}\right)_{k},\left(F_{1}\right)_{k}\right)$ is fully faithful. Since $F_{0}$ is $\tau$-epi if and only if $\left(F_{0}\right)_{k}$ is surjective for each $k \geq 0$, the statement follows from Proposition 8.15.

Corollary 8.29 The model structure on DblCat induced by the simplicially surjective topology $\tau$ on Cat coincides with the model structure obtained by transferring the diagram categorical structure across the vertical categorification-vertical nerve adjunction $c_{v} \dashv N_{v}$. The transfer across $c_{v} \dashv N_{v}$ is completely analogous to the transfer across $c_{h} \dashv N_{h}$ in Section 7.6.

Proof From Propositions 8.24 and 8.28 we see that the weak equivalences and fibrations of the two model structures coincide.

Remark 8.30 By Example 8.14, every double category is fibrant in the $\tau$-model structure.

Remark 8.31 We can now easily construct a cofibrant replacement $\mathbb{E}$ for a double category $\mathbb{B}$ in the $\tau$-model structure. Let $\mathbb{E}_{0}$ be the $\tau$-projective category associated to $\mathbb{B}_{0}$ with projection $K_{0}:=L$ as in the proof of Proposition 8.26. Then $\mathbb{E}$ and $K$ as defined in Remark 8.19 are a cofibrant replacement for $\mathbb{B}$ in the $\tau$-model structure. 
Proposition 8.32 Let $F$ be a $\tau$-equivalence. Then $B F$, as in Definition 5.12, is a weak homotopy equivalence of topological spaces.

Proof By Proposition 8.24, $F$ is a $\tau$-equivalence if and only if $\left(N_{v} F\right)_{k}$ is an equivalence of categories for each $k \geq 0$. Since $\left(\left(N_{v} F\right)_{k}\right)_{\ell}=\left(N_{d} F\right)_{k \ell}$, we see that $\left(N_{d} F\right)_{k *}$ is a weak equivalence of simplicial sets for each $k \geq 0$. Hence $\operatorname{diag}\left(N_{d} F\right)$ is a weak equivalence of simplicial sets, and $B F=\left|\operatorname{diag}\left(N_{d} F\right)\right|$ is a weak homotopy equivalence.

Remark 8.33 For each $m \in \mathbb{N}$, the assignment

$$
\mathbf{C} \mapsto K_{m}(\mathbf{C}):=\left\{\{F: \mathbf{D} \longrightarrow \mathbf{C}\} \mid F_{k} \text { surjective for all } 0 \leq k \leq m\right\}
$$

is a basis for a Grothendieck topology $\tau_{m}$ on Cat. We obtain a $\tau_{m}$-model structure as above, though $\tau_{m}$-equivalences will of course not necessarily be weak homotopy equivalences of classifying spaces.

\subsection{Model structure from the categorically surjective basis}

A functor is said to be categorically surjective if it is surjective on objects and full. It is straightforward to check that a basis for a Grothendieck topology on Cat is given by declaring a covering family to be a single categorically surjective functor. We call this topology $\tau^{\prime}$. In this section we study the model structure on DblCat induced by $\tau^{\prime}$. In Section 9 we show that this model structure is the model structure on DblCat viewed as a category of algebras over a 2 -monad.

As before we start with a characterization of the $\tau^{\prime}$-epi functors. We will use this to prove a 2 -out-of-3 property for the $\tau^{\prime}$-equivalences.

Proposition 8.34 A functor $p: \mathbf{E} \longrightarrow \mathbf{B}$ is $\tau^{\prime}$-epi if and only if there is a subcategory $\mathbf{H} \longrightarrow \mathbf{E}$ such that $\left.p\right|_{\mathbf{H}}: \mathbf{H} \longrightarrow \mathbf{B}$ is surjective on objects and full. Thus, a $\tau^{\prime}-$ epi functor is not necessarily categorically surjective.

Proof Suppose that $p$ is $\tau^{\prime}$-epi. Then by Proposition 8.5 and Remark 8.6 there is a commutative square

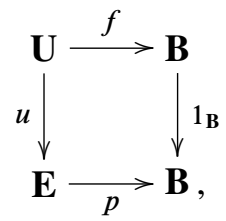


where $f$ is surjective on objects and full. For each pair of objects $x, y$ in $\mathbf{U}$, we have a commutative triangle:

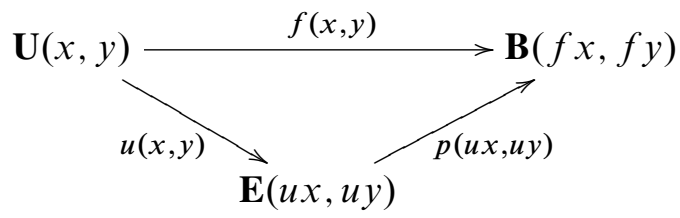

Since $f(x, y)$ is surjective, so is $p(u x, u y)$. Let $\mathbf{H}=\operatorname{im}(u)$. Thus, $\left.p\right|_{\mathbf{H}}$ is surjective on objects and full.

Conversely, let $\mathbf{H}^{\ell} \longrightarrow \mathbf{E}$ be a subcategory such that $\left.p\right|_{\mathbf{H}}$ is surjective on objects and full, and let $g: \mathbf{X} \longrightarrow \mathbf{B}$ be any functor. Consider the commutative diagram

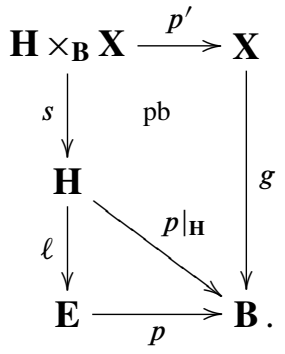

Then $p^{\prime}$ is surjective on objects and full since $\left.p\right|_{\mathbf{H}}$ is. Further, $g p^{\prime}=p \ell s$. By Proposition 8.5 and Remark 8.6, it follows that $p$ is $\tau^{\prime}$-epi.

Even though the $\tau^{\prime}$-epi functors do not coincide with the categorically surjective functors, they do give rise to the same projective objects.

Corollary 8.35 A category $\mathbf{P}$ is $\tau^{\prime}$-projective if and only if it is projective with respect to categorically surjective functors.

Proof We use the same notation as in Definition 8.10. If $\mathbf{P}$ is $\tau^{\prime}$-projective, then $\mathbf{P}$ is projective with respect to categorically surjective functors because every categorically surjective functor is $\tau^{\prime}$-epi by Proposition 8.34. For the converse, suppose $\mathbf{P}$ is projective with respect to categorically surjective functors, and suppose $G$ is $\tau^{\prime}$-epi. Then by Proposition 8.34 again, there exists an inclusion $\ell: \mathbf{Q}^{\prime} \longrightarrow \mathbf{Q}$ such that $G \ell$ is a categorically surjective functor. Thus there exists an $F^{\prime}$ such that $G \ell F^{\prime}=H$. If we let $F=\ell F^{\prime}$ then we see that $\mathbf{P}$ is $\tau^{\prime}$-projective.

Even better, we can characterize the $\tau^{\prime}$-projective objects. 
Proposition 8.36 A category is $\tau^{\prime}$-projective if and only if it is a free category on a directed graph.

Proof Let $\mathbf{C}$ be a free category on a directed graph $\Gamma$; we show that $\mathbf{C}$ is $\tau^{\prime}-$ projective. Suppose $G: \mathbf{Q} \longrightarrow \mathbf{R}$ is $\tau^{\prime}$-epi and $H: \mathbf{C} \longrightarrow \mathbf{R}$ is a functor. Let $\mathbf{Q}^{\prime} \subseteq \mathbf{Q}$ be a subcategory such that $\left.G\right|_{\mathbf{Q}^{\prime}}: \mathbf{Q}^{\prime} \longrightarrow \mathbf{R}$ is surjective on objects and full. Let $U$ : Cat $\longrightarrow$ Graph denote the forgetful functor. Then there is a map of directed graphs which makes the following diagram commute:

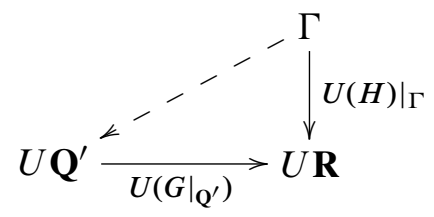

and induces a functor $F$ such that

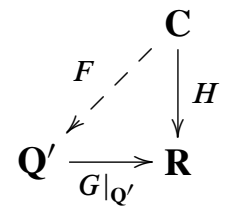

commutes. Hence the diagram

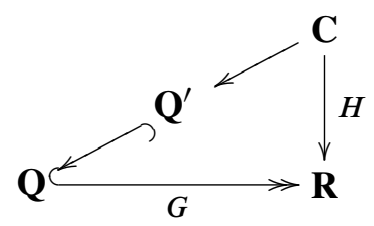

commutes and $\mathbf{C}$ is $\tau^{\prime}$-projective.

For the converse, suppose $\mathbf{P}$ is a $\tau^{\prime}$-projective category. Let $\mathbf{Q}$ be the free category on the underlying directed graph of $\mathbf{P}$ with the identity arrows omitted. Note that every arrow of $\mathbf{Q}$ is either a path $\left\langle f_{1}, \ldots, f_{n}\right\rangle$ of nonidentity arrows $f_{i}$ in $\mathbf{P}$ or an empty path \langle\rangle$_{A}$ (forming the identity arrow $1_{A}$ on the object $A$ in $\mathbf{Q}$ ). We define an identityon-objects functor $G: \mathbf{Q} \longrightarrow \mathbf{P}$ by $G\left(\langle\rangle_{A}\right)=1_{A}$ and $G\left(\left\langle f_{1}, \ldots, f_{n}\right\rangle\right)=f_{n} \circ \cdots \circ f_{1}$. The functor $G$ is clearly categorically surjective.

Consider the diagram

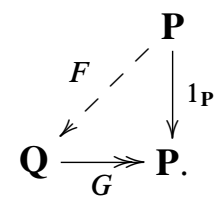


Since $\mathbf{P}$ is $\tau^{\prime}$-projective, there exists a functor $F$ which makes the diagram commute as indicated. When $F(f)=\left\langle f_{1}, \ldots, f_{n}\right\rangle$, we say that $F(f)$ has length $n$. Note that since $F$ is a functor, $F\left(1_{A}\right)$ has length 0 . By the commutativity of the diagram, the length of $F(f)$ is greater than or equal to 1 for any nonidentity arrow $f$.

Now let $f$ be any nonidentity arrow in $\mathbf{P}$, and suppose $F(f)=\left\langle f_{1}, \ldots, f_{n}\right\rangle$ as well as $F\left(f_{i}\right)=\left\langle f_{i 1}, \ldots, f_{i m_{i}}\right\rangle$. Since $G F=1_{\mathbf{P}}$, we know that $f=f_{n} \circ \cdots \circ f_{1}$. Since $F$ is a functor, we also know that $F(f)=F\left(f_{n}\right) \circ \cdots \circ F\left(f_{1}\right)=\left\langle f_{11}, \ldots, f_{n m_{n}}\right\rangle$. Thus

$$
\left\langle f_{1}, \ldots, f_{n}\right\rangle=\left\langle f_{11}, \ldots, f_{n m_{n}}\right\rangle .
$$

Since all the $f_{i}$ and $f_{i j}$ are nonidentity arrows, it follows that $m_{i}=1$ and $f_{i 1}=f_{i}$ for all $i \in\{1, \ldots, n\}$. Summarizing, if $F(f)=\left\langle f_{1}, \ldots, f_{n}\right\rangle$, then $F\left(f_{i}\right)$ has length 1 for each $i$.

Let $\mathbf{C}$ be the free category on the directed graph with the objects of $\mathbf{P}$ as vertices and as edges those arrows $f$ of $\mathbf{P}$ for which the length of $F(f)$ is 1 . By the argument above, the functor $F$ factors through a functor $\widetilde{F}: \mathbf{P} \longrightarrow \mathbf{C}$ as in the diagram

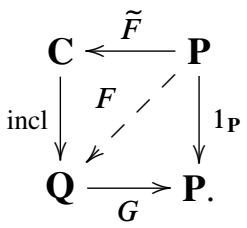

Let $\widetilde{G}$ be the restriction of $G$ to $\mathbf{C}$. It is obvious that $\widetilde{G} \widetilde{F}=1_{\mathbf{P}}$.

Now consider the other composition, $\widetilde{F} \widetilde{G}$. This is obviously the identity on objects. For a morphism $\left\langle f_{1}, \ldots, f_{n}\right\rangle$ in $\mathbf{C}$, we have

$$
\begin{aligned}
\tilde{F} \widetilde{G}\left(\left\langle f_{1}, \ldots, f_{n}\right\rangle\right) & =\widetilde{F}\left(f_{n} \circ \cdots \circ f_{1}\right) \\
& =\widetilde{F}\left(f_{n}\right) \circ \cdots \circ \widetilde{F}\left(f_{1}\right) \\
& =\left\langle f_{n}\right\rangle \circ \cdots \circ\left\langle f_{1}\right\rangle \\
& =\left\langle f_{1}, \ldots, f_{n}\right\rangle,
\end{aligned}
$$

where the second to last equality follows from the fact that the $f_{i}$ are edges in the graph on which $\mathbf{C}$ is free, in other words $\widetilde{F}\left(f_{i}\right)$ has length 1 . So $\widetilde{F} \widetilde{G}\left(\left\langle f_{1}, \ldots, f_{n}\right\rangle\right)=$ $\left\langle f_{1}, \ldots, f_{n}\right\rangle$. We conclude that $\mathbf{P} \cong \mathbf{C}$, so $\mathbf{P}$ is a free category on a directed graph.

Proposition 8.37 If a functor $p: \mathbf{A} \longrightarrow \mathbf{B}$ is $\tau^{\prime}-$ epi, then $p_{k}: \mathbf{A}_{k} \longrightarrow \mathbf{B}_{k}$ is surjective for all $k \geq 0$. 
Proof By Proposition 8.5 and Remark 8.6 there exists a functor $f$ surjective on objects and full such that $1_{\mathbf{B}} \circ f=p \circ u$ for some $u$. Since $f_{k}=p_{k} \circ u_{k}$ is surjective, so is $p_{k}$.

Proposition 8.38 If a double functor $F: \mathbb{A} \longrightarrow \mathbb{B}$ is a $\tau^{\prime}$-equivalence, then for every $k \geq 0$ the functor

$$
\left(\left(F_{0}\right)_{k},\left(F_{1}\right)_{k}\right):\left(\left(\mathbb{A}_{0}\right)_{k},\left(\mathbb{A}_{1}\right)_{k}\right) \longrightarrow\left(\left(\mathbb{B}_{0}\right)_{k},\left(\mathbb{B}_{1}\right)_{k}\right)
$$

is an equivalence of categories.

Proof Since $F$ is fully faithful, $\left(\left(F_{0}\right)_{k},\left(F_{1}\right)_{k}\right)$ is fully faithful for all $k \geq 0$ by Proposition 8.23. Since $F$ is essentially $\tau^{\prime}$-surjective, $s \circ \bar{F}_{0}$ is $\tau^{\prime}$-epi and hence $\left(s \circ \bar{F}_{0}\right)_{k}=(s)_{k} \circ\left(\bar{F}_{0}\right)_{k}$ is surjective for all $k \geq 0$ by Proposition 8.37. Remark 8.9 then implies that $\left(\left(F_{0}\right)_{k},\left(F_{1}\right)_{k}\right)$ is essentially surjective for all $k \geq 0$.

Lemma 8.39 Suppose $\mathbb{A} \stackrel{F}{\longrightarrow} \mathbb{B} \stackrel{G}{\longrightarrow} \mathbb{C}$ are double functors and two of $G F, G$, or $F$ are $\tau^{\prime}$-equivalences. Then the third double functor is fully faithful.

Proof By Proposition 8.38 the vertical nerves of the two $\tau^{\prime}$-equivalences are levelwise equivalences of categories. Hence the vertical nerve of the third double functor is also levelwise an equivalence of categories, and in particular levelwise fully faithful. By Proposition 8.23, this implies that the third functor is fully faithful.

Lemma 8.40 Suppose $\mathbb{A} \stackrel{F}{\longrightarrow} \mathbb{B} \stackrel{G}{\longrightarrow} \mathbb{C}$ are double functors and $G F$ and $F$ are $\tau^{\prime}$-equivalences. Then $G$ is essentially $\tau^{\prime}$-surjective.

Proof We need to show that $s \circ \bar{G}_{0}$ is $\tau^{\prime}$-epi. Let $\mathbf{H}_{F} \subseteq\left(\mathbb{P}_{F}\right)_{0}$ and $\mathbf{H}_{G F} \subseteq\left(\mathbb{P}_{G F}\right)_{0}$ be subcategories such that $\left.s \circ \bar{F}_{0}\right|_{\mathbf{H}_{F}}$ and $\left.s \circ \overline{(G F)}_{0}\right|_{\mathbf{H}_{G F}}$ are surjective on objects and full. Define a full subcategory $\mathbf{H}_{G}$ of $\left(\mathbb{P}_{G}\right)_{0}=\mathbb{B}_{0} \times_{\mathbb{C}_{0}}$ iso $(\mathbb{C})_{1}$ by applying $F_{0}$ to the first coordinate of $\mathbf{H}_{G F}$ as follows. For any object $\left(a, c \cong G_{0} F_{0} a\right)$ in $\mathbf{H}_{G F}$, we have an object $\left(F_{0} a, c \cong G_{0}\left(F_{0} a\right)\right)$ in $\mathbf{H}_{G}$. For any morphism

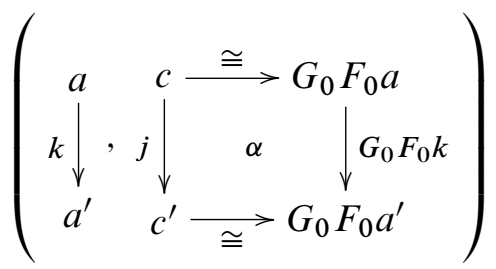


in $\mathbf{H}_{G F}$ we have a morphism

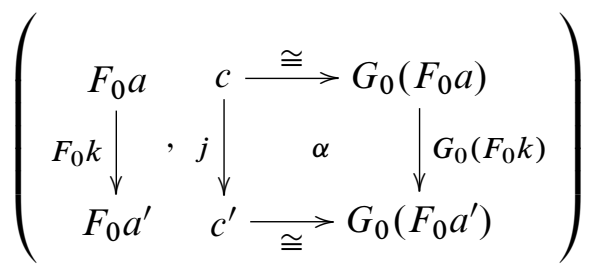

in $\mathbf{H}_{G}$. Then we see as follows that $\left.s \circ \bar{G}_{0}\right|_{\mathbf{H}_{G}}: \mathbf{H}_{G} \rightarrow \mathbb{C}_{0}$ is surjective on objects and full. If $c \in \mathbb{C}_{0}$, there exists an object $\left(a, c \cong G_{0} F_{0} a\right)$ in $\mathbf{H}_{G F}$, with $\left(F_{0} a, c \stackrel{\cong}{\rightrightarrows} G_{0}\left(F_{0} a\right)\right)$ in $\mathbf{H}_{G}$ and $s \circ \bar{G}_{0}\left(\left(F_{0} a, c \cong G_{0} F_{0} a\right)\right)=c$. So $\left.s \circ \bar{G}_{0}\right|_{\mathbf{H}_{G}}$ is surjective on objects. If $c \stackrel{j}{\rightarrow} c^{\prime}$ is a morphism in $\mathbb{C}_{0}$ and $\left(F_{0} a, c \stackrel{\cong}{\rightrightarrows} G_{0}\left(F_{0} a\right)\right)$ and $\left(F_{0} a^{\prime}, c^{\prime} \cong G_{0}\left(F_{0} a^{\prime}\right)\right)$ are objects of $\mathbf{H}_{G}$, then there exists a morphism

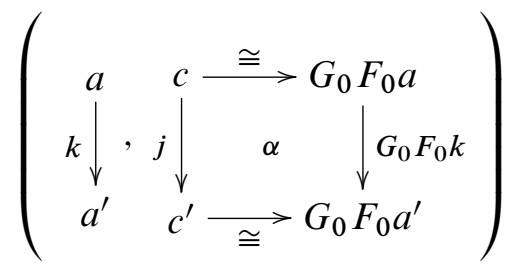

in $\mathbf{H}_{G F}$ which gives rise to a morphism

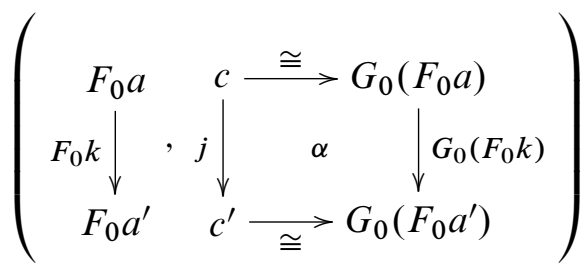

in $\mathbf{H}_{G}$ that maps to $j$ under $\left.s \circ \bar{G}_{0}\right|_{\mathbf{H}_{G}}$. We conclude that $\left.s \circ \bar{G}_{0}\right|_{\mathbf{H}_{G}}$ is surjective on objects and full and therefore $s \circ \bar{G}_{0}$ is $\tau^{\prime}$-epi. This implies that $G$ is essentially $\tau^{\prime}$-surjective.

Lemma 8.41 Suppose $\mathbb{A} \stackrel{F}{\longrightarrow} \mathbb{B} \stackrel{G}{\longrightarrow} \mathbb{C}$ are double functors and $G F$ and $G$ are $\tau^{\prime}$-equivalences. Then $F$ is essentially $\tau^{\prime}$-surjective.

Proof We need to show that $s \circ \bar{F}_{0}$ is $\tau^{\prime}$-epi. Let $\mathbf{H}_{G F} \subseteq\left(\mathbb{P}_{G F}\right)_{0}$ be a subcategory such that $\left.s \circ \overline{G F}_{0}\right|_{\mathbf{H}_{G F}}$ is surjective on objects and full. Define a full subcategory $\mathbf{H}_{F}$ of $\left(\mathbb{P}_{F}\right)_{0}=\mathbb{A}_{0} \times_{\mathbb{B}_{0}}$ iso $(\mathbb{B})_{1}$ with object set

$$
\operatorname{Obj} \mathbf{H}_{F}:=\left\{\left(a, b \stackrel{\cong}{\rightrightarrows} F_{0} a\right) \mid\left(a, G_{0}\left(b \stackrel{\cong}{\rightrightarrows} F_{0} a\right)\right) \in \operatorname{Obj} \mathbf{H}_{G F}\right\} .
$$

Then we can see as follows that $\left.s \circ \bar{F}_{0}\right|_{\mathbf{H}_{F}}: \mathbf{H}_{F} \rightarrow \mathbb{B}_{0}$ is surjective on objects and full. If $b \in \mathbb{B}_{0}$, then $G_{0} b \in \mathbb{C}_{0}$, and there is an object $\left(a, G_{0} b \stackrel{\cong}{\rightarrow} G_{0} F_{0} a\right) \in \mathbf{H}_{G F}$, 
because $\left.s \circ(\overline{G F})_{0}\right|_{\mathbf{H}_{G F}}$ is surjective on objects. However, $\left(\left(G_{0}\right)_{0},\left(G_{1}\right)_{0}\right)$ is fully faithful, that is, $G$ restricted to the objects of $\mathbb{B}$ and the horizontal morphisms of $\mathbb{B}$ is a fully faithful functor of categories. So there is a unique isomorphism $b \cong F_{0} a$ whose image under $G$ is $G_{0} b \stackrel{\cong}{\rightrightarrows} G_{0} F_{0} a$. Hence, $\left(a, b \cong F_{0} a\right) \in \mathbf{H}_{F}$ and this object maps to $b$ under $\left.s \circ \bar{F}_{0}\right|_{\mathbf{H}_{F}}$. We conclude that $\left.s \circ \bar{F}_{0}\right|_{\mathbf{H}_{F}}$ is surjective on objects.

Moreover, if $b \stackrel{j}{\rightarrow} b^{\prime}$ is a morphism in $\mathbb{B}_{0}$ and $\left(a, b \cong F_{0} a\right)$ and $\left(a^{\prime}, b^{\prime} \cong F_{0} a^{\prime}\right)$ are objects of $\mathbf{H}_{F}$, then $G_{0} j$ is a morphism of $\mathbb{C}_{0}$, and since $\left.s \circ(\overline{G F})_{0}\right|_{\mathbf{H}_{G F}}$ is full, there is a morphism of the form

$$
\left(\begin{array}{cccc}
a & G_{0} b \stackrel{G(\cong)}{\longrightarrow} G_{0} F_{0} a \\
k \downarrow & G_{0} j \mid & \alpha & G_{0} F_{0} k \\
a^{\prime} & G_{0} b^{\prime} & \underset{G(\cong)}{\longrightarrow} & G_{0} F_{0} a^{\prime}
\end{array}\right)
$$

in $\mathbf{H}_{G F}$. However, the functor $\left(\left(G_{0}\right)_{1},\left(G_{1}\right)_{1}\right)$ is fully faithful, so there is a unique square $\beta$, such that

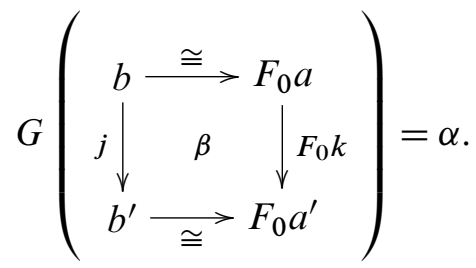

Moreover, $\beta$ is also horizontally invertible. Hence

$$
\left(\begin{array}{cccc}
a & b \stackrel{\cong}{ } F_{0} a \\
k \downarrow & j \mid & \beta & \downarrow_{0} k \\
\downarrow & \downarrow & & F_{0} \\
a^{\prime} & b^{\prime} & \cong & F_{0} a^{\prime}
\end{array}\right)
$$

is a morphism in $\mathbf{H}_{F}$ which maps to $j$ under $s \circ \bar{F}_{0}$. We conclude that $\left.s \circ \bar{F}_{0}\right|_{\mathbf{H}_{F}}$ is surjective on objects and full, so $s \circ \bar{F}_{0}$ is $\tau^{\prime}$-epi and $F$ is essentially $\tau^{\prime}$-surjective. $\square$

Lemma 8.42 Suppose that $\mathbb{A} \stackrel{F}{\longrightarrow} \mathbb{B} \stackrel{G}{\longrightarrow} \mathbb{C}$ are double functors, and $F$ and $G$ are $\tau^{\prime}$-equivalences. Then $G \circ F$ is essentially $\tau^{\prime}$-surjective.

Proof We need to show that $s \circ(\overline{G F})_{0}$ is $\tau^{\prime}$-epi. Let $\mathbf{H}_{F} \subseteq\left(\mathbb{P}_{F}\right)_{0}$ and $\mathbf{H}_{G} \subseteq\left(\mathbb{P}_{G}\right)_{0}$ be subcategories such that $\left.s \circ \bar{F}_{0}\right|_{\mathbf{H}_{F}}$ and $\left.s \circ \bar{G}_{0}\right|_{\mathbf{H}_{G}}$ are surjective on objects and full. 
Let $\mathbf{H}_{G F}$ be the full subcategory of $\left(\mathbb{P}_{G F}\right)_{0}=\mathbb{A}_{0} \times \mathbb{C}_{0}$ iso $(\mathbb{C})_{1}$, with objects

$$
\begin{aligned}
\operatorname{Obj} \mathbf{H}_{G F}:= & \left\{\left(a, c \stackrel{\cong}{\rightrightarrows} G_{0} b \cong G_{0} F_{0} a\right) \mid\right. \\
& \left.\left(b, c \stackrel{\cong}{\rightrightarrows} G_{0} b\right) \in \mathbf{H}_{G},\left(a, b \cong F_{0} a\right) \in \mathbf{H}_{F}\right\} .
\end{aligned}
$$

Suppose that $c \in \mathbb{C}_{0}$, then there are objects $\left(b, c \cong G_{0} b\right) \in \mathbf{H}_{G}$ and $\left(a, b \cong F_{0} a\right) \in \mathbf{H}_{F}$, since $\left.s \circ \bar{G}_{0}\right|_{\mathbf{H}_{G}}$ and $\left.s \circ \bar{F}_{0}\right|_{\mathbf{H}_{F}}$ are surjective on objects. So $\left(a, c \stackrel{\cong}{\rightarrow} G_{0} b \stackrel{\cong}{\rightrightarrows} G_{0} F_{0} a\right) \in$ $\mathbf{H}_{G F}$, and this object maps to $c$ under $\left.s \circ(\overline{G F})_{0}\right|_{\mathbf{H}_{G F}}$.

Next, suppose that $c \stackrel{j}{\rightarrow} c^{\prime}$ is a morphism of $\mathbb{C}_{0}$ and that $\left(a, c \stackrel{\cong}{\rightarrow} G_{0} b \stackrel{\cong}{\rightarrow} G_{0} F_{0} a\right)$ and $\left(a^{\prime}, c^{\prime} \cong G_{0} b^{\prime} \cong G_{0} F_{0} a^{\prime}\right)$ are objects of $\mathbf{H}_{G F}$. Then there exist morphisms

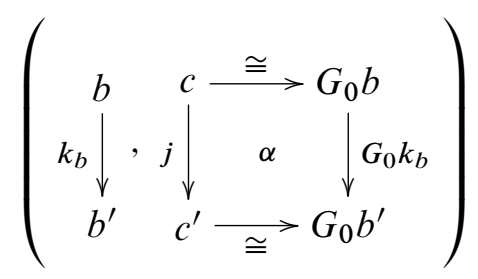

in $\mathbf{H}_{G}$, and

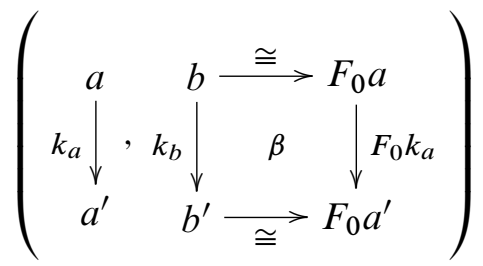

in $\mathbf{H}_{F}$, and therefore

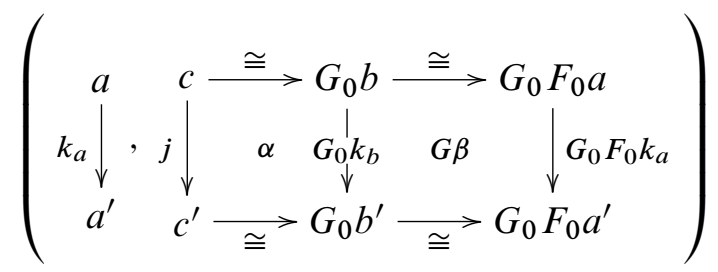

is a morphism of $\mathbf{H}_{G F}$ that maps to $c \stackrel{j}{\rightarrow} c^{\prime}$ under $\left.s \circ(\overline{G F})_{0}\right|_{\mathbf{H}_{G F}}$. So we have proved that $\left.s \circ(\overline{G F})_{0}\right|_{\mathbf{H}_{G F}}$ is surjective on objects and full. We conclude $s \circ(\overline{G F})_{0}$ is $\tau^{\prime}$-epi and $G F$ is essentially $\tau^{\prime}$-surjective.

The previous four lemmas are summarized in the following theorem.

Theorem 8.43 The class we $\left(\tau^{\prime}\right)$ of $\tau^{\prime}$-equivalences has the 2-out-of-3 property.

Proposition 8.44 Cat has enough $\tau^{\prime}$-projectives. 
Proof Suppose $\mathbf{C}$ is a category. Let $\mathbf{P}$ be the free category on the underlying directed graph of $\mathbf{C}$. This is $\tau^{\prime}$-projective by Proposition 8.36. The functor $\mathbf{P} \longrightarrow \mathbf{C}$, which is the identity on objects and defined by composition on paths of morphisms, is surjective on objects and full, so it is $\tau^{\prime}$-epi. Thus Cat has enough $\tau^{\prime}$-projectives.

Theorem 8.45 The categorically surjective topology $\tau^{\prime}$ determines a model structure (Cat (Cat), we $\left(\tau^{\prime}\right)$, fib $\left.\left(\tau^{\prime}\right), \operatorname{cof}\left(\tau^{\prime}\right)\right)$.

Proof The category Cat(Cat) is complete and cocomplete by Theorem 4.1. The class of $\tau^{\prime}$-equivalences has the 2-out-of-3 property by Theorem 8.43 and Cat has enough $\tau^{\prime}$-projectives by Proposition 8.44, so we can apply Theorem 8.1.

Remark 8.46 By Example 8.14, every double category is fibrant in the $\tau^{\prime}$-model structure.

Proposition 8.47 A double category $\mathbb{X}$ is cofibrant in the $\tau^{\prime}$-model structure if and only if $\mathbb{X}_{0}$ is a free category on a directed graph.

Proof By Corollary 8.18 a double category is cofibrant if and only if $\mathbb{X}_{0}$ is projective with respect to $\tau^{\prime}$-epi functors. But by Proposition $8.36, \mathbb{X}_{0}$ is projective with respect to $\tau^{\prime}$-epis if and only if it is a free category on a directed graph.

Remark 8.48 We can now easily construct a cofibrant replacement $\mathbb{E}$ for a double category $\mathbb{B}$ in the $\tau^{\prime}$-model structure. Let $\mathbb{E}_{0}$ be the free category on the underlying directed graph of $\mathbb{B}_{0}$, and $K_{0}: \mathbb{E}_{0} \longrightarrow \mathbb{B}_{0}$ the functor which is the identity on objects and composition on paths of morphisms. Then $\mathbb{E}_{0}$ is $\tau^{\prime}$-projective, and $K_{0}$ is a $\tau^{\prime}$-epimorphism as in the proof of Proposition 8.44. Then $\mathbb{E}$ and $K$ as defined in Remark 8.19 are a cofibrant replacement for $\mathbb{B}$ in the $\tau^{\prime}$-model structure.

As an immediate consequence of Propositions 8.24 and 8.38, we see that every $\tau^{\prime}-$ equivalence is a $\tau$-equivalence. This also follows from Remark 8.20, since the categorically surjective $\tau^{\prime}$-topology is contained in the simplicially surjective $\tau$-topology. An interesting question is whether or not a condition slightly stronger than simplicial surjectivity but also slightly weaker than categorical surjectivity would give rise to a model structure with weak equivalences between those of the $\tau^{\prime}$-structure and the $\tau$-structure. For example, such a condition on a functor is to be $U$-split. However, this condition recovers the $\tau^{\prime}$-topology instead of something new. In fact, this condition only gives a different basis for the $\tau^{\prime}$-topology which will be of use in Section 9 . 
Definition 8.49 Let $U$ : Cat $\longrightarrow$ Graph be the forgetful functor from categories to directed graphs. We say that a functor $p$ is $U$-split if there exists a morphism $q$ of directed graphs such that $(U p) \circ q=\mathrm{id}$.

Lemma 8.50 A functor $p: \mathbf{E} \longrightarrow \mathbf{B}$ is $U$-split if and only if there is a subcategory $\mathbf{H} \longrightarrow \mathbf{E}$ such that $\left.p\right|_{\mathbf{H}}: \mathbf{H} \longrightarrow \mathbf{B}$ is surjective on objects and full.

Proof Suppose $p$ is $U$-split. Then there exists a morphism of directed graphs $q$ such that $U p \circ q=$ id. Let $\mathbf{H}$ be the full subcategory $\mathbf{E}$ whose objects are in the image of $q$. Then $\left.p\right|_{\mathbf{H}}$ is surjective on objects and full, as one sees using the directed graph section $q$.

Conversely, suppose there exists a subcategory $\mathbf{H}$ of $\mathbf{E}$ such that $\left.p\right|_{\mathbf{H}}$ is categorically surjective. Then $\left.p\right|_{\mathbf{H}}$ is $U$-split, and id $=U(p \mid \mathbf{H}) \circ q=U p \circ q$ so that $p$ is also $U$-split.

\section{Proposition 8.51 The assignment}

$$
\mathbf{C} \mapsto L(\mathbf{C}):=\{\{F: \mathbf{D} \longrightarrow \mathbf{C}\} \mid F \text { is } U-\text { split }\}
$$

is a basis for the $\tau^{\prime}$-topology on Cat.

Proof We omit the proof that this is a basis.

Recall that a sieve is a covering sieve in the topology induced by a basis if and only if it contains a covering family from the basis. If $S$ is a $\tau^{\prime}$-covering sieve, it contains a categorically surjective functor, and hence a $U$-split functor by Lemma 8.50, so that $S$ is also a covering sieve in the topology induced by $L$.

Conversely, suppose $S$ is a sieve on $\mathbf{B} \in \mathbf{C a t}$ containing a $U-$ split functor $p$ : $\mathbf{E} \longrightarrow \mathbf{B}$ and $\left.p\right|_{\mathbf{H}}$ is categorically surjective. Then $p \circ i \in S$ for the inclusion $i: \mathbf{H} \longrightarrow \mathbf{E}$, and $S$ is a covering sieve in the $\tau^{\prime}$-topology.

\subsection{Model structure from the trivial topology}

On the underlying category of any 2 -category $\mathcal{K}$ with finite limits and finite colimits there is the trivial model structure as proved by Lack [60] using pseudo limits. A weak equivalence (respectively fibration) in this model structure is a morphism $f: A \longrightarrow B$ such that $\mathcal{K}(E, f): \mathcal{K}(E, A) \longrightarrow \mathcal{K}(E, B)$ is a weak equivalence (respectively fibration) for all $E$ in the categorical model structure on Cat. Thus $f$ is a weak equivalence if and only if there is a morphism $g: B \longrightarrow A$ such that $g f$ and $f g$ are isomorphic via $2-$ cells to the respective identities. A morphism $f$ is a fibration, or isofibration, 
if and only if for all morphisms $a: E \longrightarrow A$ and $b: E \longrightarrow B$ and any invertible 2-cell $\beta: b \cong f a$, there exists a morphism $a^{\prime}: E \longrightarrow A$ and an invertible 2-cell $\alpha: a^{\prime} \cong a$ with $f a^{\prime}=b$ and $f \alpha=\beta$ (the notion of isofibration at the end of Section 7.1 is a special case of this). If the $2-$ category $\mathcal{K}$ is merely a 1 -category, then the trivial model structure agrees with the usual trivial model structure: the weak equivalences are exactly the isomorphisms and all morphisms are both fibrations and cofibrations. The trivial model structure on the underlying category of a 2 -category $\mathcal{K}$ is compatible with the Cat-enrichment as proved in [60].

Thus DblCat admits three trivial model structures, depending on whether we take as 2-cells the horizontal natural transformations, the vertical natural transformations, or only trivial 2-cells. When we say trivial model structure on DblCat we mean the one arising from the 2-category DblCat $_{\mathbf{h}}=$ Cat(Cat) which has horizontal natural transformations as its 2 -cells.

The following theorem summarizes Section 7 of Everaert-Kieboom-Van der Linden's paper [32] and Theorem 3.3 of Lack's paper [60] applied to $\mathcal{K}=$ Cat(C) to conclude that the $\tau_{\text {triv }}$-model structure coincides with the trivial model structure on the underlying category of the 2-category Cat $(\mathbf{C})$.

Theorem 8.52 Let $\mathbf{C}$ be a finitely complete category such that Cat $(\mathbf{C})$ is finitely complete and finitely cocomplete. Let $\tau_{\text {triv }}$ denote the trivial topology ${ }^{7}$ on $\mathbf{C}$. Then the following hold.

(1) A morphism $p$ in $\mathbf{C}$ is $\tau_{\text {triv }}$-epi if and only if there exists a morphism $q$ such that $p q=\mathrm{id}$.

(2) An internal functor $F$ is an acyclic $\tau_{\text {triv }}-$ fibration if and only if there exists an internal functor $G$ such that $F G=$ id and $G F \cong$ id.

(3) Every object of $\mathbf{C}$ is $\tau_{\text {triv }}$-projective, and hence $\mathbf{C}$ has enough projectives.

(4) Assume for the rest of this theorem that the $\tau_{\text {triv }}$-equivalences have the 2-out-of-3 property. Then we have the $\tau_{\text {triv }}$-model structure on Cat $(\mathbf{C})$ of Theorem 8.1.

(5) Every object of Cat(C) is fibrant and cofibrant.

(6) The weak equivalences in the $\tau_{\text {triv }}$-model structure are precisely the equivalences in the 2-category Cat $(\mathbf{C})$.

(7) The $\tau_{\text {triv }}$-model structure coincides with the trivial model structure of [60] on the underlying category of the 2 -category $\mathcal{K}=\operatorname{Cat}(\mathbf{C})$.

\footnotetext{
${ }^{7}$ In the trivial topology the only covering sieve on an object is the maximal sieve.
} 


\section{Proof}

(1) This follows from Proposition 8.5.

(2) This is Proposition 7.1 of [32], and it follows from (1) along with Proposition 8.15 .

(3) This follows immediately from (1).

(4) The model structure follows from (3) and the hypotheses we have made.

(5) Every object is fibrant by Example 8.14, and every object is cofibrant by (2).

(6) Since every object is fibrant and cofibrant, the weak equivalences are precisely the homotopy equivalences. In Section 3 of [32], a cocylinder on Cat(C) is given such that two internal functors are homotopic if and only if they are naturally isomorphic. Hence, the weak equivalences are precisely the equivalences.

(7) By Theorem 3.3 of [60], the weak equivalences in the trivial model structure on Cat $(\mathbf{C})$ are the equivalences. By 3.4 of [60], the acyclic fibrations in the trivial model structure are the morphisms in (2). Since the classes of weak equivalences and acyclic fibrations in the two model structures are the same, we conclude that the two model structures coincide.

Remark 8.53 The assumption that the $\tau_{\text {triv }}$-equivalences have the 2 -out-of-3 property can be removed by proving directly that the $\tau_{\text {triv }}$-equivalences are the equivalences in the 2-category Cat(C) using (1) and fully faithfulness.

The trivial model structure on a category of internal categories is much like the Strøm structure of [79]. This analogy is made precise in Section 7 of [32].

We finish this section by comparing the categorical model structure on Cat with the trivial model structure on DblCat that arises from DblCat $_{\mathbf{h}}$. Our comparison uses the horizontal embedding $\mathbb{H}$ of Cat into DblCat, which is the same as the embedding

$$
\text { Cat }(\text { Set }) \longrightarrow \text { Cat }(\text { Cat })
$$

induced by the embedding Set $\longrightarrow$ Cat .

Proposition 8.54 The functor $(\mathbf{H}-)_{0}$ : DblCat $\longrightarrow$ Cat maps the weak equivalences and fibrations of the trivial model structure on DblCat to weak equivalences and fibrations in the categorical model structure on Cat. In particular, $(\mathbf{H}-)_{0}$ is a right Quillen functor. 
Proof The functor $(\mathbf{H}-)_{0}$ preserves weak equivalences because it is the underlying functor of the 2-functor $\mathbf{H}$ : DblCat $_{\mathbf{h}} \longrightarrow$ Cat , which maps equivalences to equivalences.

To prove that $(\mathbf{H}-)_{0}$ preserves fibrations, we use the characterization of isofibrations (in a 2-category in general, and in Cat in particular) at the beginning of Section 8.4. If $F$ is a double functor that is an isofibration, and $\beta: b \Longrightarrow(\mathbf{H} F)_{0} \circ a$ is a natural isomorphism in Cat, then we obtain the required $\alpha$ by applying $\mathbb{H}$ to the lifting problem, and then applying $(\mathbf{H}-)_{0}$ to the solution $\alpha^{\prime}$ of the new lifting problem in DblCat.

Corollary 8.55 The adjunction

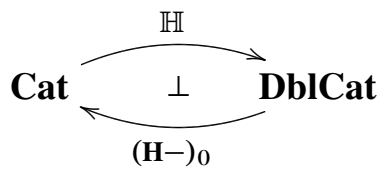

is a Quillen adjunction.

Proof This follows immediately from Proposition 8.54.

Proposition 8.56 The functor $\mathbb{H}$ : Cat $\longrightarrow$ DblCat preserves and reflects weak equivalences, fibrations, and cofibrations. In other words, a functor $F$ is a weak equivalence (respectively fibration, respectively cofibration) in the categorical model structure on Cat if and only if $\mathbb{H} F$ is a weak equivalence (respectively fibration, respectively cofibration) in the trivial model structure on DblCat that arises from the 2-category DblCat $_{\mathbf{h}}$. As a consequence, the trivial model structure on DblCat from DblCat $\mathbf{t}_{\mathbf{h}}$ extends the categorical model structure on Cat as a horizontally embedded subcategory.

Proof The 2-functor $\mathbb{H}$ : Cat $\longrightarrow$ DblCat is fully faithful in the 2-categorical sense, so it preserves and reflects equivalences (=weak equivalences).

The functor $\mathbb{H}$ preserves and reflects isofibrations because of the characterization of isofibrations at the beginning of Section 8.4 and the fact that horizontal natural transformations between functors $\mathbb{E} \longrightarrow \mathbb{H} \mathbf{C}$ are in bijective correspondence with natural transformations between the underlying 1-functors.

Cofibrations are preserved by $\mathbb{H}$ by Corollary 8.55 . The functor $\mathbb{H}$ reflects cofibrations as follows. If $F$ is a functor such that the double functor $\mathbb{H} F$ is a cofibration, and $G$ 
is an acyclic fibration in Cat, then any diagram in DblCat

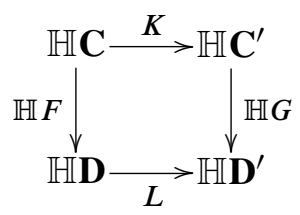

admits a lift, as $\mathbb{H} G$ is an acyclic fibration by the above. Since $\mathbb{H}$ is fully faithful, this lift gives us a lift in Cat. Hence the functor $F$ is a cofibration as well.

After this discussion of model structures on DblCat as a category of internal categories in Section 8, we now turn to a model structure on DblCat as a category of algebras and show that this model structure is the same as the categorically surjective model structure. We will make use of the trivial model structure on the underlying category of the 2-category Cat(Graph).

\section{A model structure for DblCat as the 2-category of algebras for a 2-monad}

Every 2-category of strict algebras over a 2-monad $T$ with rank (that is, which preserves $\alpha$-filtered colimits for some $\alpha$ ) on a locally finitely presentable 2-category $\mathcal{K}$ admits a canonical cofibrantly generated Cat-enriched model structure as proved by Lack [60]. It is obtained by transferring the trivial model structure on the 2category $\mathcal{K}$ described in Section 8.4. A strict morphism of strict $T$-algebras is a weak equivalence (respectively fibration) if and only if its underlying morphism is an equivalence (respectively isofibration). We prove that the model structure induced by the categorically surjective topology $\tau^{\prime}$ can be recovered in this way.

The interest in having these two different descriptions of the $\tau^{\prime}$-model structure lies in the fact that they allow a characterization of the flexible double categories (Corollary 9.4 and Remark 9.7). We will see that the cofibrant replacement in the $\tau^{\prime}$-model structure of Remark 8.48 is left 2-adjoint to the inclusion of strict algebras, strict morphisms, and 2-cells into strict algebras, pseudo morphisms, and 2-cells. In particular, the cofibrant replacement in Remark 8.48 coincides with the cofibrant replacement in the algebra structure of Lack [60]. Another interesting aspect of the two descriptions of the $\tau^{\prime}$-model structure is that DblCat provides a good setting for comparing Lack's categorical model structure on 2-Cat [58;59] to a model structure on algebras over a 2-monad.

Recall that the adjunction Graph $\dashv$ Cat induces a Cartesian monad $M$ on the category Graph of small directed graphs. These directed graphs are nonreflexive; a choice 
of distinguished arrows called identities is not part of the data. The algebras of $M$ are precisely the small categories. A Cartesian monad is a monad whose underlying functor preserves pullbacks and whose unit and multiplication are Cartesian natural transformations, that is, each of their naturality squares is a pullback square. Since $M$ is Cartesian, it induces a $2-$ monad $\bar{M}$ on Cat(Graph), the 2-category of internal categories in Graph. The strict algebras for this 2 -monad $\bar{M}$ are pairs $\left(\mathbb{D}_{0}, \mathbb{D}_{1}\right)$ of $M$-algebras with source, target, unit, and composition maps compatible with the $M$-algebra structure. Thus, the strict $\bar{M}$-algebras are precisely double categories. Similarly, strict morphisms and 2-cells for strict $\bar{M}$-algebras are double functors and horizontal natural transformations. The 2-category of strict $\bar{M}$-algebras, strict morphisms, and 2-cells is

$$
\bar{M}-\operatorname{Alg}_{\mathrm{s}}=\operatorname{Cat}(\mathbf{C a t})=\text { DblCat }_{\mathbf{h}} .
$$

The term algebra will always mean strict algebra, so we occasionally leave off the adjective strict.

Let $U:$ Cat $\longrightarrow$ Graph be the forgetful functor. The functor $U$ induces a 2-functor

$$
\bar{U}: \operatorname{Cat}(\mathbf{C a t}) \longrightarrow \operatorname{Cat}(\mathbf{G r a p h})
$$

which coincides with the forgetful 2-functor

$$
\bar{M}-\mathrm{Alg}_{\mathrm{s}} \longrightarrow \mathrm{Cat}(\mathbf{G r a p h}) .
$$

An internal category in Graph is a (nonreflexive) double graph $\mathbb{E}$ with a category structure on $(\mathrm{Obj} \mathbb{E}, \operatorname{Hor} \mathbb{E})$ and on $(\operatorname{Ver} \mathbb{E}, \mathrm{Sq} \mathbb{E})$, in other words horizontal compositions are defined in the double graph $\mathbb{E}$ but vertical compositions are not. There are no vertical identity 1 -arrows in $\mathbb{E}$, and no vertical identity squares on horizontal 1 -morphisms.

Theorem 9.1 The model structure on DblCat induced by the $2-$ monad $\bar{M}$ is the $\tau^{\prime}$-model structure.

Proof First we prove that the weak equivalences are the same. Note that a double functor $G$ is fully faithful if and only if $\bar{U} G$ is fully faithful as in Definition 8.8. A double functor $G$ is a weak equivalence as a morphism of algebras if and only if $\bar{U} G$ is a weak equivalence in the trivial model structure on Cat(Graph), which is the case if and only if $\bar{U} G$ is fully faithful and there exists a morphism $q$ of directed graphs such that $U\left(s \circ \bar{G}_{0}\right) \circ q=\mathrm{id}_{\mathbb{B}_{0}}$ by Definition 8.8 and Theorem 8.52 (1). That is equivalent to $G$ being fully faithful and $s \circ \bar{G}_{0}$ being $U$-split, which is precisely the definition of 
weak equivalence in the $\tau^{\prime}$-model structure using Proposition 8.34 and Lemma 8.50. Hence the weak equivalences coincide.

Similarly, a double functor $G$ is a fibration as a morphism of algebras if and only if $\bar{U} G$ is a fibration in the trivial model structure on Cat(Graph), which is the case if and only if there exists a morphism $q$ of directed graphs such that $\left(U\left(r_{G}\right)_{0}\right) \circ q=$ id in Diagram (19), which is the case if and only if $\left(r_{G}\right)_{0}$ is $U$-split. This is equivalent to $G$ being a fibration in the $\tau^{\prime}$-model structure. Hence the fibrations coincide.

Corollary 9.2 The categorically surjective $\tau^{\prime}$-model structure is cofibrantly generated and admits an enrichment as a Cat-enriched model category.

For a $2-$ monad $T$ on $\mathcal{K}$ as above, let $T-\mathrm{Alg}_{\mathrm{s}}$ denote the 2 -category of strict $T-$ algebras, strict morphisms, and 2-cells. As usual, we denote by $T$-Alg the 2-category of strict $T$-algebras, pseudo morphisms, and 2-cells. As proved by Blackwell-KellyPower [8], the inclusion $T-\mathrm{Alg}_{\mathrm{s}} \longrightarrow T$-Alg admits a left 2-adjoint denoted $A \mapsto A^{\prime}$. The counit component $q: A^{\prime} \longrightarrow A$ is a strict morphism, and if $q$ admits a section in $T-\mathrm{Alg}_{\mathrm{s}}$, then $A$ is called flexible. The flexible algebras are the closure under flexible colimits of the free algebras. Strict morphisms from $A^{\prime}$ to $B$ are in bijective correspondence with pseudo morphisms from $A$ to $B$.

Theorem 9.3 (Theorem 4.12 of Lack [60]) The cofibrant objects of $T$-Alg $\lg _{\mathrm{s}}$ are precisely the flexible algebras; in particular, any algebra of the form $A^{\prime}$ is cofibrant, and is thus a cofibrant replacement for A. Every free algebra is flexible.

Corollary 9.4 The cofibrant objects in the $\tau^{\prime}$-model structure are precisely the flexible double categories. In particular, a double category $\mathbb{X}$ is flexible if and only if $\mathbb{X}_{0}$ is a free category on a directed graph.

Proof This follows from Theorem 9.1, Theorem 9.3, and Proposition 8.47.

We next show that the cofibrant replacement in the $\tau^{\prime}$-model structure of Remark 8.48 is the same as the left 2 -adjoint $\mathbb{A} \mapsto \mathbb{A}^{\prime}$ to the inclusion $\bar{M}-\mathrm{Alg}_{\mathrm{s}} \longrightarrow \bar{M}-\mathrm{Alg}$. Our method is to verify that the cofibrant replacement has the same 2 -universal property as $\mathbb{A}^{\prime}$. For this we need to identify the pseudo morphisms of strict $\bar{M}$-algebras.

Proposition 9.5 The pseudo morphism between strict $\bar{M}$-algebras are the pseudo double functors which are strict in the horizontal direction, but weak in the vertical direction. 
Proof Let $\mathbb{A}$ and $\mathbb{B}$ be strict algebras for the 2 -monad $\bar{M}$, in other words, $\mathbb{A}$ and $\mathbb{B}$ are internal categories in Graph equipped with internal functors $a: \bar{M}(\mathbb{A}) \longrightarrow \mathbb{A}$, and $b: \bar{M}(\mathbb{B}) \longrightarrow \mathbb{B}$ defining the vertical composition. More specifically, $a_{0}$ and $b_{0}$ define the vertical compositions of paths of vertical arrows, and $a_{1}$ and $b_{1}$ define the vertical composition of vertical paths of squares.

A pseudo morphism $(F, \varphi): \mathbb{A} \longrightarrow \mathbb{B}$ consists of an internal functor $F: \mathbb{A} \longrightarrow \mathbb{B}$ in Graph and an invertible internal transformation

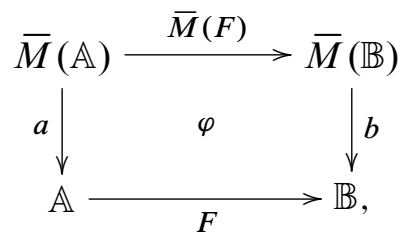

given by a morphism of graphs $\varphi:(\bar{M}(\mathbb{A}))_{0} \longrightarrow \mathbb{B}_{1}$ which satisfies the coherence conditions from Blackwell-Kelly-Power [8]. It follows from the identity coherence condition that $\varphi$ sends every object of $\mathbb{A}$ to the corresponding horizontal identity arrow. To every path $\left\langle u_{1}, \ldots, u_{n}\right\rangle$ of compatible vertical arrows in $\mathbb{A}, \varphi$ assigns a horizontally invertible square in $\mathbb{B}$ denoted by

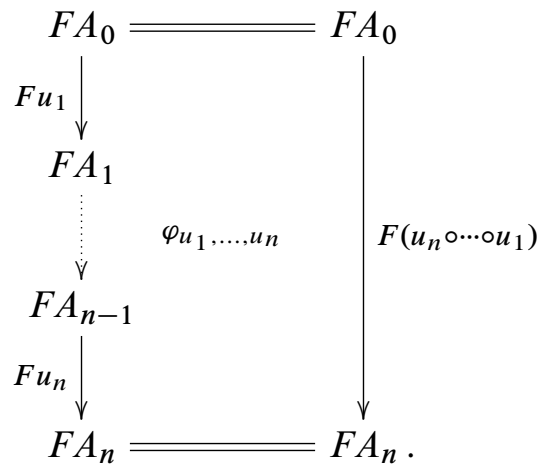

It follows from the second coherence axiom that for any path of paths

$$
\left\langle\left\langle u_{11}, \ldots, u_{1 m_{1}}\right\rangle,\left\langle u_{21}, \ldots, u_{2 m_{2}}\right\rangle, \ldots,\left\langle u_{n 1}, \ldots, u_{n m_{n}}\right\rangle\right\rangle,
$$

the pasting in Figure 5 is equal to $\varphi_{u_{11}, \ldots, u_{1 m_{1}}, \ldots, u_{n 1}, \ldots, u_{n m_{n}}}$.

Note that the coherence conditions imply $\varphi$ is completely determined by its components $\varphi_{u_{1}, u_{2}}$ for composable vertical arrows $u_{1}$ and $u_{2}$ in $\mathbb{A}$. These $\varphi_{u_{1}, u_{2}}$ are the composition coherence isomorphisms for the underlying vertical pseudo functor of $F$. The internal natural transformation $\varphi$ associates to the empty path on $A$ the unit coherence isomorphism of the underlying vertical pseudo functor of $F$. The coherence 


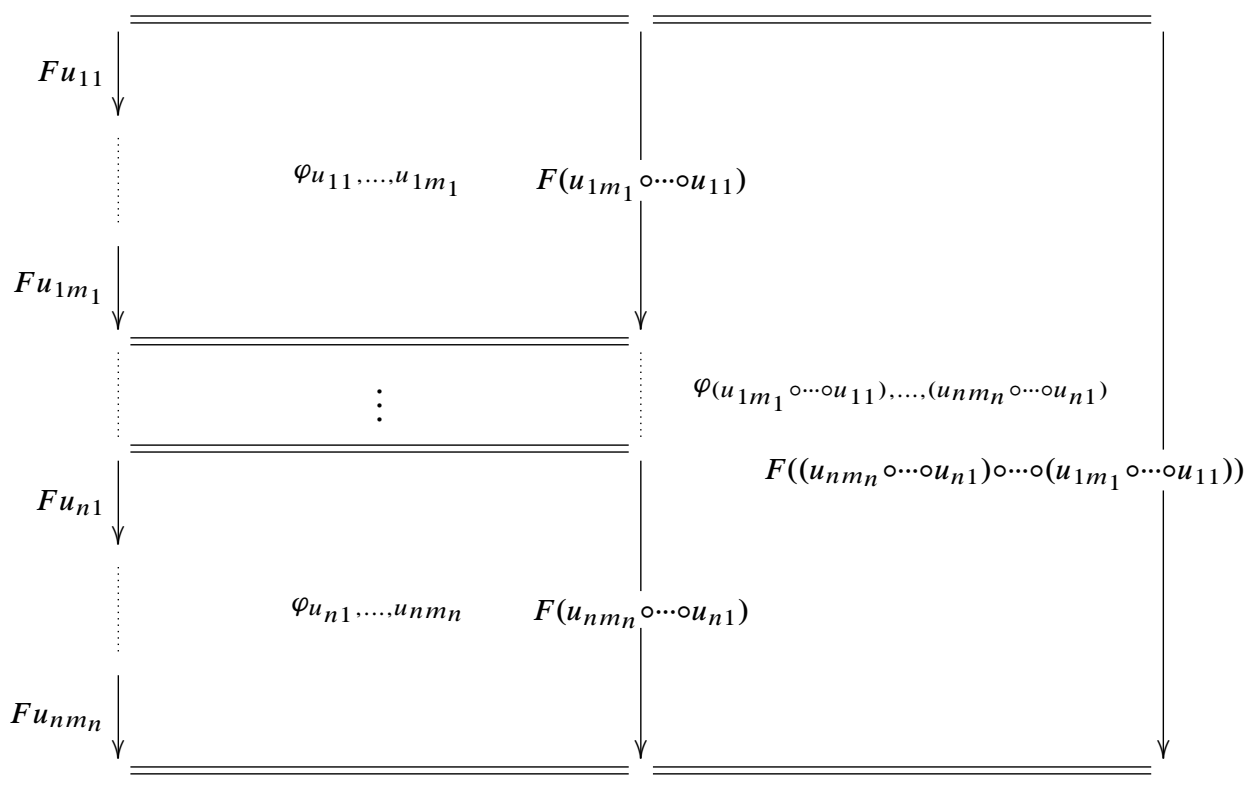

Figure 5: Pasting of associativity coherence squares

conditions on $\varphi$ contain the coherence conditions for the coherence isomorphisms of a pseudo functor.

Conversely, given a pseudo double functor (weak in the vertical direction) the natural isomorphism $\varphi$ is defined in terms of the coherence isomorphisms.

Proposition 9.6 The cofibrant replacement in the $\tau^{\prime}$-model structure of Remark 8.48 is isomorphic to the left 2 -adjoint $\mathbb{A} \mapsto \mathbb{A}^{\prime}$ to the inclusion $\bar{M}-\mathrm{Alg}_{\mathrm{s}} \longrightarrow \bar{M}-\mathrm{Alg}$.

Proof Let $Q$ denote the cofibrant replacement functor defined on objects in Remark 8.48. Our task is to present a natural isomorphism of categories

$$
\bar{M}-\operatorname{Alg}_{\mathrm{s}}(Q \mathbb{A}, \mathbb{B}) \cong \bar{M}-\mathrm{Alg}(\mathbb{A}, \mathbb{B})
$$

for strict $\bar{M}$-algebras (double categories) $\mathbb{A}$ and $\mathbb{B}$. 
The category $(Q \mathbb{A})_{0}$ is the free category on the underlying directed graph of $\mathbb{A}_{0}$, and the category $(Q \mathbb{A})_{1}$ is the pullback in Cat

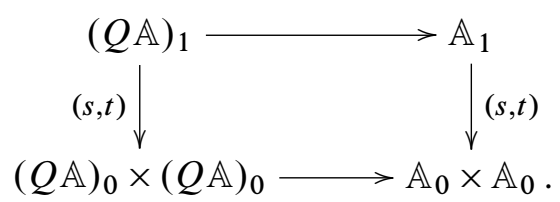

In particular, squares of $Q \mathbb{A}$ (the morphisms of $\left.(Q \mathbb{A})_{1}\right)$ have the form

$$
\left(\left\langle u_{1}, \ldots, u_{m}\right\rangle, \alpha,\left\langle v_{1}, \ldots, v_{n}\right\rangle\right),
$$

where $\alpha$ is a square in $\mathbb{A}$ with the vertical morphism $u=u_{m} \circ \cdots u_{1}$ as its horizontal source and the vertical morphism $v=v_{n} \circ \cdots \circ v_{1}$ as its horizontal target.

Given a double functor $G: Q \mathbb{A} \longrightarrow \mathbb{B}$, we may define a pseudo morphism

$$
(F, \phi): \mathbb{A} \longrightarrow \mathbb{B}
$$

as follows. On objects, $F(A)=G(A)$; on horizontal morphisms, $F(f)=G(f)$; on vertical morphisms, $F(u)=G(\langle u\rangle)$; and on squares, $F(\alpha)=G(\langle u\rangle, \alpha,\langle v\rangle)$, where $u$ is the horizontal source of $\alpha$ and $v$ is the horizontal target of $\alpha$. Further, $\phi$ has components $\phi_{u_{1}, \ldots, u_{m}}=G\left(\left\langle u_{1}, \ldots, u_{m}\right\rangle, i_{u_{m} \circ \cdots \circ u_{1}}^{h},\left\langle u_{m} \circ \cdots \circ u_{1}\right\rangle\right)$ (where $i_{u_{m} \circ \cdots \circ u_{1}}^{h}$ is the horizontal identity square on the vertical morphism $\left.u_{m} \circ \cdots \circ u_{1}\right)$.

Given a pseudo morphism $(F, \varphi): \mathbb{A} \longrightarrow \mathbb{B}$, we may define a double functor

$$
G: Q \mathbb{A} \longrightarrow \mathbb{B}
$$

as follows. On objects, $G(A)=F(A)$; on horizontal morphisms, $G(f)=F(f)$; on vertical morphisms,

$$
G\left(\left\langle u_{1}, \ldots, u_{m}\right\rangle\right)=F\left(u_{m}\right) \circ \cdots \circ F\left(u_{1}\right)
$$

and on squares

$$
G\left(\left\langle u_{1}, \ldots, u_{m}\right\rangle, \alpha,\left\langle v_{1}, \ldots, v_{n}\right\rangle\right)=\left[\begin{array}{lll}
\phi_{u_{1}, \ldots, u_{m}} & F \alpha & \left(\varphi_{v_{1}, \ldots, v_{m}}\right)^{-1}
\end{array}\right] .
$$

It is straightforward to see that these two procedures are inverse to each other, and define a bijection on the object sets of Equation (20). We extend this bijection to an isomorphism of categories. If $\theta: G_{1} \Longrightarrow G_{2}$ is a horizontal natural transformation of double functors, then we define $\lambda: F_{1} \Longrightarrow F_{2}$ by $\lambda_{A}=\theta_{A}$ and

$$
\lambda_{\left\langle u_{1}, \ldots, u_{m}\right\rangle}=\left[\begin{array}{lll}
\left(\varphi_{1}\right)_{u_{1}, \ldots, u_{m}} & \theta_{u_{m} \circ \cdots \circ u_{1}} & \left(\varphi_{2}\right)_{u_{1}, \ldots, u_{m}}^{-1}
\end{array}\right] .
$$


Conversely, if $\lambda: F_{1} \Longrightarrow F_{2}$ is a horizontal natural transformation of pseudo morphisms, then we define $\theta: G_{1} \Longrightarrow G_{2}$ by $\theta_{A}=\lambda_{A}$ and $\theta_{u}=\lambda\langle u\rangle$.

Naturality can be easily verified.

Remark 9.7 Proposition 9.6 allows us to give an alternative proof of Corollary 9.4. In fact, if $\mathbb{A}$ is a cofibrant object in the $\tau^{\prime}$-model structure, the map $\varnothing \longrightarrow \mathbb{A}$ has the left lifting property with respect to the counit component $q: Q \mathbb{A} \longrightarrow \mathbb{A}$, which is an acyclic fibration by Lemma 5.14 of Everaert-Kieboom-Van der Linden [32]. It follows easily that $q$ has a section, and thus $\mathbb{A}$ is flexible. Conversely, if $\mathbb{A}$ is flexible, then $q$ has a section $p$. In the diagram below, let $f$ be an acyclic fibration in the $\tau^{\prime}$-model structure and $h$ any map.

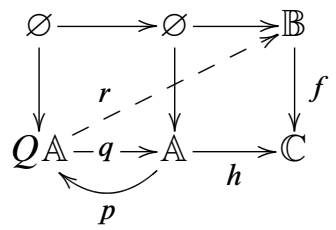

Since $Q \mathbb{A}$ is cofibrant, there is a map $r: Q \mathbb{A} \longrightarrow \mathbb{B}$ with $f r=h q$. Hence the map $r p: \mathbb{A} \longrightarrow \mathbb{B}$ satisfies $f r p=h q p=h$. This shows that $\varnothing \longrightarrow \mathbb{A}$ is a cofibration; that is, $\mathbb{A}$ is cofibrant.

The categorical model structure on 2-Cat of Lack [58; 59] has weak equivalences the strict 2-functors that are biequivalences, fibrations those strict 2-functors with the equivalence lifting property (as defined in [59], not [58]), and cofibrations those strict 2-functors whose underlying functor has the left lifting property with respect to functors that are surjective on objects and full. We can compare this with the $\tau^{\prime}$-model structure as follows.

Proposition 9.8 Consider 2-Cat vertically embedded in DblCat. If a 2-functor is a cofibration in the $\tau^{\prime}$-model structure on DblCat, then it is a cofibration in the categorical model structure on 2-Cat. A 2-category is cofibrant in the categorical model structure on 2-Cat if and only if it is cofibrant in the $\tau^{\prime}$-model structure on DblCat. Thus a 2-category is flexible in the sense of Lack [58] if and only if it is flexible as an algebra over the $2-$ monad $\bar{M}$.

Proof Suppose $G$ is a 2 -functor such that $\mathbb{V} G$ is a cofibration in the $\tau^{\prime}$-model structure on DblCat. Then $(\mathbb{V} G)_{0}$ has the left lifting property with respect to $\tau^{\prime}-$ functors by Proposition 8.17 . This implies that $(\mathbb{V} G)_{0}$ has the left lifting property with respect to all functors that are surjective on objects and full by Proposition 8.34. The 
underlying functor of $G$ is $(\mathbb{V} G)_{0}$, so $G$ is a cofibration in the categorical structure on 2-Cat.

A 2-category is cofibrant in the categorical structure on 2-Cat if and only if its underlying category is projective with respect to all functors that are surjective on objects and full. But this coincides with cofibrant 2-categories in the $\tau^{\prime}$-model structure on DblCat by Corollary 8.18 and Corollary 8.35 .

Remark 9.9 Our characterization of flexible double categories extends Lack's characterization of flexible 2-categories as those 2-categories with underlying category a free category on a graph (Theorem 4.8 (iv) of [58]). See Corollary 9.4 and Proposition 9.8 .

The sets of weak equivalences with source and target 2-categories in the two model structures have nontrivial intersection, but neither set of weak equivalences is contained in the other. The $\mathbb{V}$-image of a biequivalence is not necessarily essentially $\tau^{\prime}$-surjective, though it is fully faithful. For example, consider the inclusion $h:\{0\} \longrightarrow\{0 \cong 1\}$. Then $h$ is a biequivalence, and the only object of $\left(\mathbb{P}_{\mathbb{V} h}\right)_{0}$ in Definitions 8.3 and 8.8 is $(0,0 \stackrel{\mathrm{id} 0}{\longrightarrow} 0)$. Then $s \circ \overline{\mathbb{V}}_{0}$ cannot be surjective on objects, as its target has two objects. By Proposition 8.34, the functor $s \circ \overline{\mathbb{V}}_{0}$ is not $\tau^{\prime}$-epi. Thus $\mathbb{V} h$ is not essentially $\tau^{\prime}$-surjective.

For a reason why the left adjoint $\mathbb{V}$ in Proposition 9.8 is not a left Quillen functor, consider the 2-functor $j_{1}^{\prime}$ given by inclusion of the terminal 2-category $\{1\}$ into the free-living adjoint equivalence $E^{\prime}$. The free-living adjoint equivalence $E^{\prime}$ has objects 0 and 1 . Morphisms are

$$
\begin{aligned}
& f: 0 \longrightarrow 1 \\
& g: 1 \longrightarrow 0,
\end{aligned}
$$

as well as all concatenations of $f$ and $g$. There is a unique 2-cell between every parallel pair of morphisms. In particular, every 2-cell of $E^{\prime}$ is invertible. The 2functor $j_{1}^{\prime}$ is a generating acyclic cofibration for the categorical structure on 2-Cat as described in [59]. However $\mathbb{V} j_{1}^{\prime}$ is not a cofibration in the $\tau^{\prime}$-model structure on DblCat: its underlying functor $\left(\mathbb{V} j_{1}^{\prime}\right)_{0}$ of object categories does not have the left lifting property with respect to all $\tau^{\prime}$-epimorphisms. For example, let $\mathbf{C}$ be the smallest category containing the underlying category $\left(E^{\prime}\right)_{0}=\left(\mathbb{V} E^{\prime}\right)_{0}$ of $E^{\prime}$ as well as an additional object $1^{\prime}$ and an arrow $1^{\prime} \longrightarrow 0$. The projection from $\mathbf{C}$ to $E^{\prime}$ takes 
$1^{\prime}$ to 1 and is the identity on $E^{\prime}$, hence it is $\tau^{\prime}$-epi. Then the commutative diagram

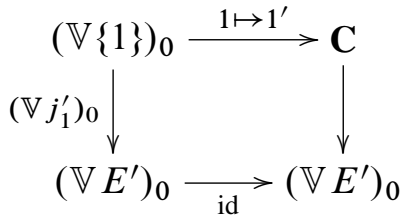

does not admit a lift. Thus, $\mathbb{V}$ in Proposition 9.8 preserves neither cofibrations nor weak equivalences.

It is interesting to note that the Cat-analogue of Theorem 9.1 does not hold. In other words, if we view Cat as the category of algebras over the 2-monad $M$ on Graph, then the associated model structure on Cat is not the model structure associated to the topology of surjective functions on Set. A covering family in a basis for this topology is a single surjective function, so that the epimorphisms for this topology are the same as the epimorphisms for the trivial topology by Proposition 8.5 and Remark 8.6, namely the surjective maps themselves. In fact, the trivial topology, simplicially surjective topology, and categorically surjective topology on Set all give rise to the categorical model structure on Cat, while the algebra structure on Cat has weak equivalences the isomorphisms of categories. When we pass to DblCat on the other hand, the three model structures associated to these three topologies become distinct, and one of them agrees with the algebra structure.

\section{Appendix: Horizontal nerves and pushouts}

Though the horizontal nerve preserves filtered colimits, it certainly does not preserve general colimits, not even pushouts. The purpose of this appendix is to explicitly describe the behavior of the horizontal nerve on pushouts in DblCat along

$$
i \otimes 1_{\mathbf{C}}: \mathbf{A} \otimes \mathbf{C} \longrightarrow \mathbf{B} \otimes \mathbf{C}
$$

where $i: \mathbf{A} \longrightarrow \mathbf{B}$ is either of the following full inclusions from Section 7.1.

$$
\begin{gathered}
c \operatorname{Sd}^{2} \Lambda^{k}[m] \longrightarrow c \operatorname{Sd}^{2} \Delta[m] \\
\{1\} \longrightarrow \mathbf{I}
\end{gathered}
$$

Theorem 10.7 is the main technical result needed for an application of Kan's Lemma on Transfer (Theorem 7.11) to transfer model structures across the adjunction $c_{h} \dashv N_{h}$ in Theorem 7.13 and Theorem 7.17. In the following, we use " $"$ " to denote set-theoretic complement. We begin with some pushouts in Cat which will aid us in our description 
of the horizontal and vertical 1-categories of the pushouts in Theorem 10.6. The squares will require an induction argument. Special pushouts in Cat have been considered by many people, for example Fritsch-Latch [36], Thomason [80] and Trnková [82].

Lemma 10.1 If $A \subseteq B$ and $D$ are sets, then the pushout in Set

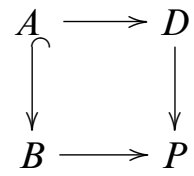

is $P=D \bigsqcup(B \backslash A)$.

Lemma 10.2 Suppose $\mathbf{A}$ is a full subcategory of $\mathbf{B}$ and

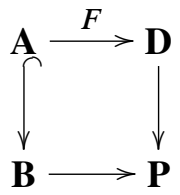

is a pushout in Cat. Then the objects of $\mathbf{P}$ are

$$
\operatorname{Obj} \mathbf{P}=\operatorname{Obj} \mathbf{D} \coprod(O b j \mathbf{B} \backslash \operatorname{Obj} \mathbf{A})
$$

and morphisms of $\mathbf{P}$ have two forms:

(1) A morphism $B_{0} \stackrel{f}{\longrightarrow} B_{1}$ with $f \in(\operatorname{Mor} \mathbf{B} \backslash \operatorname{Mor} \mathbf{A})$.

(2) A path $X_{1} \stackrel{f_{1}}{\longrightarrow} D_{1} \stackrel{d}{\longrightarrow} D_{2} \stackrel{f_{2}}{\longrightarrow} X_{2}$ where $d$ is a morphism in $\mathbf{D}$, and $f_{1}, f_{2} \in(\operatorname{Mor} \mathbf{B} \backslash \operatorname{Mor} \mathbf{A}) \cup\{$ identities on $\operatorname{Obj} \mathbf{P}\}$. If $f_{1}$ is nontrivial, then $D_{1} \in \mathbf{A}$. If $f_{2}$ is nontrivial, then $D_{2} \in \mathbf{A}$.

Proof To calculate a pushout of categories, one takes the free category on the pushout of the underlying graphs, and then mods out by the relations necessary to make the natural maps from $\mathbf{A}, \mathbf{B}, \mathbf{D}$ to the free category into functors as in Theorem 4.3. Thus the objects of $\mathbf{P}$ are

$$
\operatorname{Obj} \mathbf{D} \coprod(\operatorname{Obj} \mathbf{B} \backslash \operatorname{Obj} \mathbf{A})
$$

by Lemma 10.1. The edges of the pushout graph are

$$
\text { Mor D } \coprod(\text { Mor } \mathbf{B} \backslash \operatorname{Mor} \mathbf{A}) \text {, }
$$

again by Lemma 10.1. The free category on this consists of finite composable paths of these edges. 
Suppose

$$
P_{0} \stackrel{f_{1}}{\longrightarrow} P_{1} \stackrel{f_{2}}{\longrightarrow} P_{2} \ldots \ldots \ldots \ldots \ldots P_{k-1} \stackrel{f_{k}}{\longrightarrow} P_{k}
$$

is a morphism in the pushout $\mathbf{P}$. Then we can reduce it to the form (1) or (2) using the relations induced by $\mathbf{A}, \mathbf{B}$, and $\mathbf{D}$ as follows. Suppose $f_{j-1}$ and $f_{j+1}$ are in Mor D, while $f_{j}$ is in (Mor $\mathbf{B} \backslash$ Mor $\mathbf{A}$ ). Then $P_{j-1}$ and $P_{j}$ must be objects of $\mathbf{A}$. But by the fullness of $\mathbf{A}, f_{j}$ must be in Mor $\mathbf{A}$, and we have arrived at a contradiction. Thus no morphism of (Mor $\mathbf{B} \backslash$ Mor $\mathbf{A}$ ) can be surrounded by morphisms of $\mathbf{D}$ : there exist $0 \leq m \leq n \leq k+1$ such that for all $0 \leq j \leq m$ and all $n \leq j \leq k$ we have $f_{j} \in(\operatorname{Mor} \mathbf{B} \backslash \operatorname{Mor} \mathbf{A})$, and for all $m<j<n$ we have $f_{j} \in$ Mor $\mathbf{D}$. Next we compose the $f_{j}$ in each range, and we obtain a path of the form (1) or (2).

Remark 10.3 A morphism $f$ of $\mathbf{B}$ is in Mor $\mathbf{B} \backslash$ Mor $\mathbf{A}$ if and only if its source or target is in Obj $\mathbf{B} \backslash$ Obj $\mathbf{A}$ by the fullness of $\mathbf{A}$ in $\mathbf{B}$.

Lemma 10.4 If $A \subseteq B$ are sets and $\mathbf{C}$ and $\mathbf{D}$ are categories, then the pushout in Cat

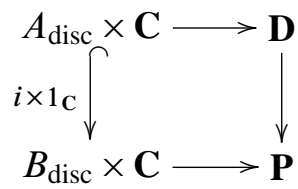

is $\mathbf{P}=\mathbf{D} \bigsqcup\left((B \backslash A)_{\text {disc }} \times \mathbf{C}\right)$. (The subscript "disc" means discrete category on a given set.)

Proof Since $B_{\text {disc }} \times \mathbf{C}=A_{\text {disc }} \times \mathbf{C} \amalg\left((B \backslash A)_{\text {disc }} \times \mathbf{C}\right)$, the pushout of the underlying graphs is

$$
\mathbf{D} \coprod\left((B \backslash A)_{\operatorname{disc}} \times \mathbf{C}\right)
$$

by Lemma 10.1. The free category on this graph, modulo the appropriate relations as in Theorem 4.3, is once again (21).

Alternatively, this Lemma also follows easily from Lemma 10.2.

Lemma 10.5 Suppose $\mathbf{A}$ is a full subcategory of $\mathbf{B}, C$ is a set, and

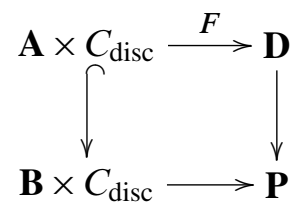


is a pushout in Cat. Then the objects of $\mathbf{P}$ are

$$
\operatorname{Obj} \mathbf{P}=\operatorname{Obj} \mathbf{D} \coprod((\operatorname{Obj} \mathbf{B} \backslash \operatorname{Obj} \mathbf{A}) \times C)
$$

and the morphisms of $\mathbf{P}$ have two forms:

(1) A morphism $\left(B_{0}, c\right) \stackrel{f}{\longrightarrow}\left(B_{1}, c\right)$ with $c$ in $C$ and $f=\left(f^{\prime}, c\right)$ in $($ Mor $\mathbf{B} \backslash \operatorname{Mor} \mathbf{A}) \times C$.

(2) A path $X_{1} \stackrel{f_{1}}{\longrightarrow} D_{1} \stackrel{d}{\longrightarrow} D_{2} \stackrel{f_{2}}{\longrightarrow} X_{2}$ where $d$ is a morphism in $\mathbf{D}$, and each of $f_{1}$ and $f_{2}$ is either in Mor $\mathbf{B} \backslash$ Mor $\mathbf{A} \times C$ or an identity morphism.

Moreover, if $f_{1}$ or $f_{2}$ is not an identity morphism in (2), then the path has one of the two respective forms

$$
\begin{aligned}
& \left(B_{1}, c_{1}\right) \stackrel{\left(f_{1}^{\prime}, c_{1}\right)}{\longrightarrow}\left(A_{1}, c_{1}\right) \stackrel{d}{\longrightarrow} D_{2} \stackrel{f_{2}}{\longrightarrow} X_{2} \\
& X_{1} \stackrel{f_{1}}{\longrightarrow} D_{1} \stackrel{d}{\longrightarrow}\left(A_{2}, c_{2}\right) \stackrel{\left(f_{2}^{\prime}, c_{2}\right)}{\longrightarrow}\left(B_{2}, c_{2}\right)
\end{aligned}
$$

where $c_{1}, c_{2} \in C, B_{1}, B_{2} \in \operatorname{Obj} \mathbf{B} \backslash \operatorname{Obj} \mathbf{A}, A_{1}, A_{2} \in \operatorname{Obj} \mathbf{A}, f_{1}^{\prime}, f_{2}^{\prime} \in \operatorname{Mor} \mathbf{B} \backslash \operatorname{Mor} \mathbf{A}$, and $d \in \operatorname{Mor} \mathbf{D}$.

Proof This follows from Lemma 10.2.

Let us recall the two full inclusions $i: \mathbf{A} \longrightarrow \mathbf{B}$ under consideration. The first case in which we are interested is the full inclusion of posets $c \operatorname{Sd}^{2} \Lambda^{k}[m] \longrightarrow c \operatorname{Sd}^{2} \Delta[m]$. Here $c$ : SSet $\longrightarrow$ Cat denotes the fundamental category functor as described in Section 6 and Sd: SSet $\longrightarrow$ SSet is the subdivision functor recalled in [39] and in Section 7.1.

The second full inclusion $i: \mathbf{A} \longrightarrow \mathbf{B}$ of interest is $\{1\} \longrightarrow \mathbf{I}$. The category $\mathbf{I}$ consists of two objects 0 and 1 and four morphisms: an isomorphism and its inverse between 0 and 1 , and the identity maps. The discrete subcategory $\{1\}$ is clearly full.

We can now give an explicit description of pushouts in DblCat along

$$
i \otimes 1_{\mathbf{C}}: \mathbf{A} \otimes \mathbf{C} \longrightarrow \mathbf{B} \otimes \mathbf{C}
$$

which we use immediately in Theorem 10.7 for the transfer. 
Theorem 10.6 Let $i: \mathbf{A} \longrightarrow$ B be either of the following full inclusions.

$$
\begin{gathered}
c \operatorname{Sd}^{2} \Lambda^{k}[m] \longrightarrow c \operatorname{Sd}^{2} \Delta[m] \\
\{1\} \longrightarrow \mathbf{I}
\end{gathered}
$$

Let $\mathbf{C}$ be a category (eg the finite ordinal $[n]$ ), and $\mathbb{D}$ a double category. Then the pushout

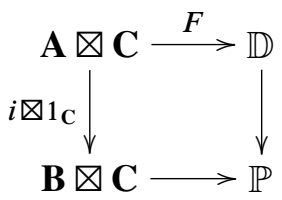

in DblCat has the following explicit description:

$$
\begin{aligned}
\operatorname{Obj} \mathbb{P}= & \text { Obj } \mathbb{D} \coprod((\operatorname{Obj} \mathbf{B} \backslash \text { Obj } \mathbf{A}) \times \text { Obj } \mathbf{C}) \\
(\mathbf{H P})_{0}= & (\mathbf{H} \mathbb{D})_{0} \coprod\left((\operatorname{Obj} \mathbf{B} \backslash \text { Obj } \mathbf{A})_{\mathrm{disc}} \times \mathbf{C}\right) \\
\operatorname{Mor}(\mathbf{V} \mathbb{P})_{0}= & \{\text { paths of the form }(1) \text { and }(2) \\
& \text { in Lemma } 10.5 \text { with } C=\text { Obj } \mathbf{C} \\
& \text { and } \left.\mathbf{D}=(\mathbf{V D D})_{0}\right\} .
\end{aligned}
$$

Squares of $\mathbb{P}$ have two forms:

(1) A square

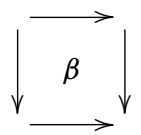

in $\mathrm{Sq}(\mathbf{B} \otimes \mathbf{C}) \backslash \mathrm{Sq}(\mathbf{A} \otimes \mathbf{C})$.

(2) A vertical path of squares

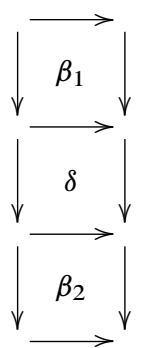

where $\delta$ is a square in $\mathbb{D}$ and each of $\beta_{1}$ and $\beta_{2}$ is either a vertical identity square (on a horizontal morphism) in $\mathbb{P}$ or is in $\mathrm{Sq}(\mathbf{B} \otimes \mathbf{C}) \backslash \mathrm{Sq}(\mathbf{A} \otimes \mathbf{C})$. Moreover, in the case of $c \mathrm{Sd}^{2} \Lambda^{k}[m] \longrightarrow c \mathrm{Sd}^{2} \Delta[m]$, the square $\beta_{1}$ is always a vertical identity square. 
Note that $\mathrm{Sq}(\mathbf{B} \otimes \mathbf{C}) \backslash \mathrm{Sq}(\mathbf{A} \otimes \mathbf{C})=$

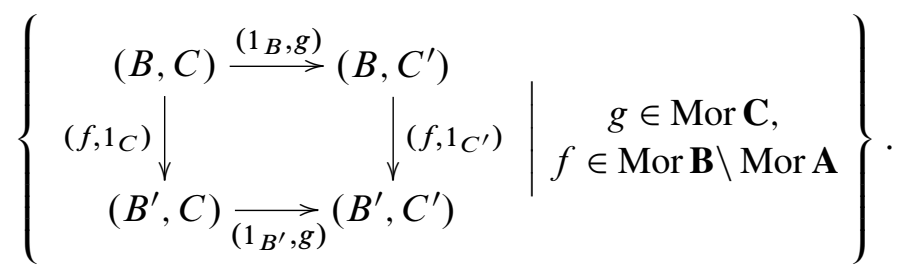

Proof We use Theorem 4.6. First we calculate the pushout $\mathbb{S}$ of the underlying double derivation schemes. The object set Obj $\mathbb{S}=\operatorname{Obj} \mathbb{P}$ is the pushout of the object sets, so (22) follows from Lemma 10.1. The horizontal and vertical 1-categories of $\mathbb{S}$ (and $\mathbb{P})$ are the pushouts of the horizontal and vertical 1-categories, so (23) follows from Lemma 10.4 and (24) follows from Lemma 10.5. By Lemma 10.1 again, the pushout of the sets of squares is

$$
\mathrm{Sq} \mathbb{S}=\mathrm{Sq} \mathbb{D} \coprod(\mathrm{Sq}(\mathbf{B} \otimes \mathbf{C}) \backslash \mathrm{Sq}(\mathbf{A} \otimes \mathbf{C})) .
$$

Thus we have calculated the pushout $\mathbb{S}$ of the underlying double derivation schemes, its horizontal and vertical 1 -categories coincide with those of $\mathbb{P}$, and they have the form claimed in the theorem. It only remains to show that the squares of $\mathbb{P}$ have the form claimed in the theorem.

The double category $\mathbb{P}$ is the free double category on the double derivation scheme $\mathbb{S}$ modulo the smallest congruence making the natural morphisms of double derivation schemes from $\mathbf{A} \otimes \mathbf{C}, \mathbf{B} \otimes \mathbf{C}$, and $\mathbb{D}$ to $\mathbb{P}$ into double functors. Squares of $\mathbb{P}$ are represented by allowable compatible arrangements in $\mathbb{S}$. To prove that squares of $\mathbb{P}$ have the form (1) or (2), it suffices to show that any allowable compatible arrangement of squares in $\mathbb{S}$ can be transformed into (1) or (2) using the relations of the congruence and the double category associativity, identity, and interchange axioms. The congruence allows us to compose squares according to the relations in the double categories $\mathbf{A} \otimes \mathbf{C}, \mathbf{B} \otimes \mathbf{C}$, and $\mathbb{D}$.

We must treat the two inclusions $i$ separately.

Let $i: \mathbf{A} \longrightarrow \mathbf{B}$ be the full inclusion $c \operatorname{Sd}^{2} \Lambda^{k}[m] \longrightarrow c \operatorname{Sd}^{2} \Delta[m]$. Recall from Section 7.1 that $c \mathrm{Sd}^{2} \Lambda^{k}[m]$ and $c \mathrm{Sd}^{2} \Delta[m]$ are respectively the posets of nondegenerate simplices of $\operatorname{Sd} \Lambda^{k}[m]$ and $\operatorname{Sd} \Delta[m]$, and that there is a morphism of the form $\left(u_{0}, \ldots, u_{p}\right) \longrightarrow\left(v_{0}, \ldots, v_{q}\right)$ in $\mathbf{B}$ if and only if

$$
\left\{u_{0}, \ldots, u_{p}\right\} \subseteq\left\{v_{0}, \ldots, v_{q}\right\} .
$$


Also, an object $\left(v_{0}, \ldots, v_{q}\right)$ of $\mathbf{B}$ is in $\mathbf{A}$ if and only all $v_{r}$ are faces of $\Lambda^{k}[m]$. Thus, we see for any path of composable morphisms in $\mathbf{B}$

$$
B_{0} \stackrel{f_{1}}{\longrightarrow} B_{1} \stackrel{f_{2}}{\longrightarrow} B_{2} \ldots \ldots \ldots B_{n-1} \stackrel{f_{n}}{\longrightarrow} B_{n}
$$

with $B_{j}$ not in $\mathbf{A}$, all $f_{\ell}$ and $B_{\ell}$ with $\ell \geq j$ are also not in $\mathbf{A}$. Thus, once a path leaves $\mathbf{A}$, it cannot return to $\mathbf{A}$. In particular, if $B \longrightarrow B^{\prime}$ is a morphism in $\mathbf{B}$ and $B$ is not in $\mathbf{A}$, then $B^{\prime}$ is also not in $\mathbf{A}$. Another useful property of $\mathbf{B}$ is that every morphism has a unique decomposition into irreducibles. These special features of the posets $\mathbf{A}$ and $\mathbf{B}$ allow us to put the squares of $\mathbb{P}$ into the desired form (1) or (2), as we do now.

Suppose $R$ is an allowable compatible arrangement of squares in $\mathbb{S}$, that is, a representative of a square in $\mathbb{P}$. If $R$ consists entirely of squares in $\mathbb{D}$, then it is equivalent to its composition in $\mathbb{D}$, so it has the form (2) and we are finished.

So suppose that $R$ contains at least one square in $\mathrm{Sq} \mathbf{B} \otimes \mathbf{C} \backslash \mathrm{Sq} \mathbf{A} \otimes \mathbf{C}$. Then $R$ has at least one vertex $(B, C)$ in $\mathbf{B} \otimes \mathbf{C}$ but not in $\mathbf{A} \otimes \mathbf{C}$, that is, $B$ is in $\mathbf{B}$ but not in $\mathbf{A}$. Any horizontal morphism in $R$ with source (respectively target) $(B, C)$ is in $\mathbf{B} \otimes \mathbf{C}$ but not in $\mathbf{A} \otimes \mathbf{C}$, as $(B, C)$ is not in $\mathbf{A} \otimes \mathbf{C}$. Thus the target (respectively source) of such a morphism has the form $\left(B, C^{\prime}\right)$ and is also in $\mathbf{B} \otimes \mathbf{C}$ but not $\mathbf{A} \otimes \mathbf{C}$. Any vertical morphism in $R$ with source $(B, C)$ is in $\mathbf{B} \otimes \mathbf{C}$ but not in $\mathbf{A} \otimes \mathbf{C}$, as $(B, C)$ is not in $\mathbf{A} \otimes \mathbf{C}$. Thus the target of such a vertical morphism is of the form $\left(B^{\prime}, C\right)$ with $B^{\prime}$ not in $\mathbf{A}$ by the special feature of the posets $\mathbf{A}$ and $\mathbf{B}$ described in the preceding paragraph. From the original vertex $(B, C)$ we traverse down a vertical morphism with source $(B, C)$ if there is one, otherwise we traverse to the right along a horizontal morphism with source $(B, C)$. In either case, we arrive at another vertex $\left(B_{1}, C_{1}\right)$ which is in $\mathbf{B} \otimes \mathbf{C}$ but not in $\mathbf{A} \otimes \mathbf{C}$. From this vertex we repeat the procedure, moving either to the right or down. We continue in this way until we reach the bottom edge of the allowable compatible arrangement $R$. We conclude that the entire bottom edge of the diagram consists of objects and horizontal morphisms in $\mathbf{B} \otimes \mathbf{C}$ but not in $\mathbf{A} \otimes \mathbf{C}$, and hence not in $\mathbb{D}$.

Each of these horizontal morphisms on the bottom edge is the bottom edge of a square in $\mathbf{B} \otimes \mathbf{C}$ but not in $\mathbf{A} \otimes \mathbf{C}$, since squares of $\mathbb{D}$ only have vertices in $\mathbb{D}$ (some objects of $\mathbb{D}$ are identified with objects of $\mathbf{A} \otimes \mathbf{C}$ ). Thus, the bottom portion of $R$ looks like Figure 6 with all squares in $\mathbf{B} \otimes \mathbf{C}$ but not in $\mathbf{A} \otimes \mathbf{C}$.

Next we factor the vertical morphisms of Figure 6 into irreducibles, which we can do since these vertical morphisms are of the form $(f, C)$ where $f$ is a morphism in $\mathbf{B}$ and $C$ is an object of $\mathbf{C}$. By the uniqueness of the factorization and the form of squares in $\mathbf{B} \otimes \mathbf{C}$, we can factor these squares at the height of the shortest one as illustrated in 


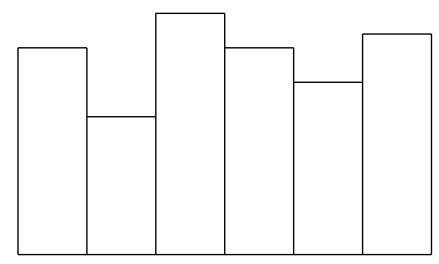

Figure 6: The bottom portion of $R$

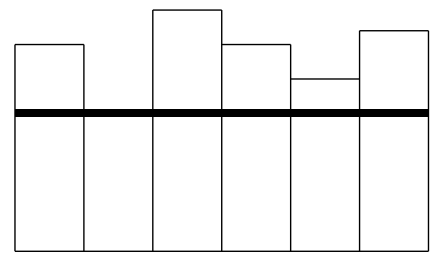

Figure 7: A factorization of the squares in Figure 6

Figure 7. We include these new horizontal morphisms into the allowable compatible arrangement $R$, and obtain a new compatible arrangement $R_{1}$. The compatible arrangement $R_{1}$ is also allowable, since the same cuts that make $R$ allowable also make $R_{1}$ allowable.

The bold horizontal line in Figure 7 is a full length cut on an allowable compatible arrangement $R_{1}$, hence it divides $R_{1}$ into two allowable compatible arrangements by Proposition 3.7. We denote the upper allowable compatible arrangement by $R_{1,1}$ and the lower allowable compatible arrangement by $R_{1,2}$. Then $R_{1,1}$ has at least one square less than $R$, since we cut off at the height of the shortest square whose bottom edge is on the bottom edge of $R$. If we argue by induction on the number of squares in an allowable compatible arrangement, we may assume that $R_{1,1}$ is equivalent to a square of the form (1) or (2). The allowable compatible arrangement $R_{1,2}$ is equivalent to a square of the form (1), as it can be composed horizontally. Finally, we compose $R_{1,1}$ with $R_{1,2}$ to conclude that $R$ is also equivalent to a compatible arrangement of the form (1) or (2).

We only need an argument for the triviality of $\beta_{1}$ whenever a compatible arrangement is equivalent to one of the form (2). Suppose $\beta_{1}$ is in $\mathrm{Sq}(\mathbf{B} \otimes \mathbf{C}) \backslash \mathrm{Sq}(\mathbf{A} \otimes \mathbf{C})$. Then its lower two vertices cannot be in $\mathbf{A} \otimes \mathbf{C}$ (for if they were, the upper two vertices must also be in $\mathbf{A} \otimes \mathbf{C}$, and the square $\beta_{1}$ would be in $\mathbf{A} \otimes \mathbf{C}$ ). Thus, the upper two vertices 
of the square $\delta$ are not in $\mathbf{A} \otimes \mathbf{C}$, a contradiction. Thus $\beta_{1}$ must be a vertical identity. This completes the proof of Theorem 10.6 for the case $c \operatorname{Sd}^{2} \Lambda^{k}[m] \longrightarrow c \operatorname{Sd}^{2} \Delta[m]$.

Now we turn to the squares in the second case. Let $i: \mathbf{A} \longrightarrow \mathbf{B}$ be the full inclusion $\{1\} \longrightarrow \mathbf{I}$ where $\mathbf{I}$ is the category with two objects 0 and 1 and an isomorphism between them. We will again argue by induction on the number of squares in the allowable compatible arrangement, but the special features of the inclusion $\{1\} \longrightarrow \mathbf{I}$ are different from those of the previous case. Note that $\mathbf{B} \otimes \mathbf{C}$ only has the four types of squares listed in Figure 8. The only vertical morphisms in $\mathbf{B} \otimes \mathbf{C}$ that are identified

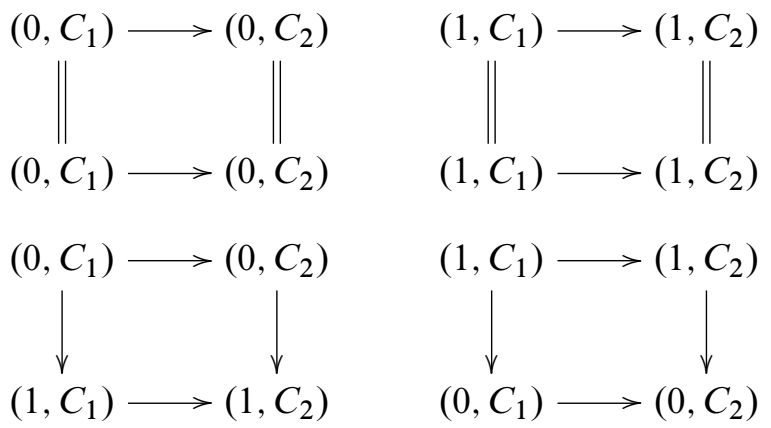

Figure 8: The four types of squares in $\mathbf{B} \otimes \mathbf{C}$

with a morphism in $\mathbb{D}$ are the trivial vertical morphisms $\operatorname{id}_{(1, C)}^{v}:(1, C) \longrightarrow(1, C)$. Suppose that any allowable compatible arrangement of squares in $\mathbb{S}$ with fewer than $n$ squares is equivalent in $\mathbb{P}$ to one of the form (1) or (2). Let $R$ be an allowable compatible arrangement of $n$ squares in $\mathbb{S}$. Since $R$ is allowable, it admits a full length cut $\mathcal{C}$ which divides $R$ into two allowable compatible arrangements each with fewer than $n$ squares. We now recombine these two smaller allowable compatible arrangements to show that $R$ is equivalent to a compatible arrangement of the form (1) or (2), but the argument is slightly different depending on whether $\mathcal{C}$ is horizontal or vertical.

Suppose the full length cut $\mathcal{C}$ is horizontal. Let $R_{1}$ and $R_{2}$ be the allowable compatible arrangements above and below $\mathcal{C}$ respectively. Since $R_{1}$ and $R_{2}$ have fewer than $n$ squares, they must be equivalent to compatible arrangements of the form (1) or (2). If $R_{1}$ and $R_{2}$ both are equivalent to compatible arrangements of the form (2), then by the fullness of $\mathbf{A}$ in $\mathbf{B}$ their vertical composite is also of the form (2), and hence $R$ is equivalent to a compatible arrangement of the form (2). If one or both of $R_{1}$ and $R_{2}$ 
has the form (1), then one can similarly conclude that $R$ is equivalent to a compatible arrangement of form (1) or (2).

Suppose the full length cut $\mathcal{C}$ is vertical. Let $Q^{\ell}$ and $Q^{r}$ be the allowable compatible arrangements to the left and to the right of $\mathcal{C}$ respectively. Since $Q^{\ell}$ and $Q^{r}$ have fewer than $n$ squares, they must be equivalent to compatible arrangements of the form (1) or (2). There are several cases to consider.

If both $Q^{\ell}$ and $Q^{r}$ are equivalent to compatible arrangements of the form (1), then their horizontal composite $R$ is clearly in $\mathbf{B} \otimes \mathbf{C}$, and hence also equivalent to a compatible arrangement of the form (1) or (2).

If $Q^{\ell}$ is equivalent to a compatible arrangement of the form (1) and $Q^{r}$ is equivalent to a compatible arrangement of the form (2), then $\beta_{1}^{r}$ and $\beta_{2}^{r}$ must be in $\mathbf{B} \otimes \mathbf{C}$ as in Figure 9. Further, the vertical morphism $k^{r}:\left(B_{2}^{r}, C\right) \longrightarrow\left(B_{3}^{r}, C\right)$ must be the
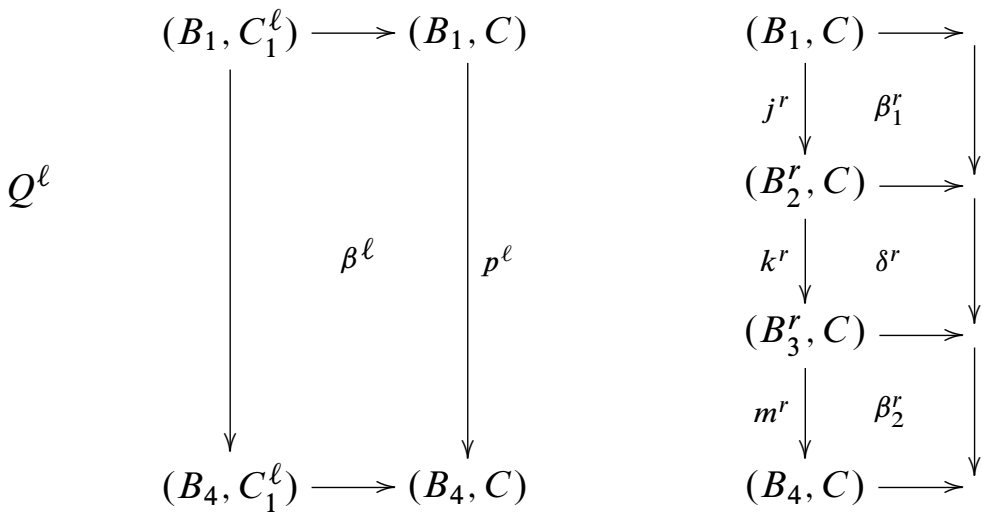

Figure 9: $Q^{\ell}$ of form (1) and $Q^{r}$ of form (2)

vertical identity $\operatorname{id}_{(1, C)}^{v}$ on the object $(1, C)$, since $k^{r}=\left(m^{r}\right)^{-1} p^{\ell}\left(j^{r}\right)^{-1}$ lies in both $\mathbf{B} \otimes \mathbf{C}$ and $\mathbb{D}$, and the only vertical morphisms in both $\mathbf{B} \otimes \mathbf{C}$ and $\mathbb{D}$ are such vertical identities. Then we can subdivide $\beta^{\ell}$ in $\mathbf{B} \otimes \mathbf{C}$ as in Figure 10. The middle square of $Q^{\ell}$ is now an identity square on a horizontal morphism in $\mathbb{D}$, and hence is also a square in $\mathbb{D}$. Finally, we horizontally compose $Q^{\ell}$ and $Q^{r}$ and use the interchange law to obtain a compatible arrangement of the form (1) or (2). Hence $R$ is equivalent to a compatible arrangement of the form (1) or (2).

Next we consider the case where $Q^{\ell}$ and $Q^{r}$ are both equivalent to compatible arrangements of the form (2) and the squares $\beta_{1}^{\ell}, \beta_{2}^{\ell}, \beta_{1}^{r}, \beta_{2}^{r}$ are in $\mathbf{B} \otimes \mathbf{C}$ as in 

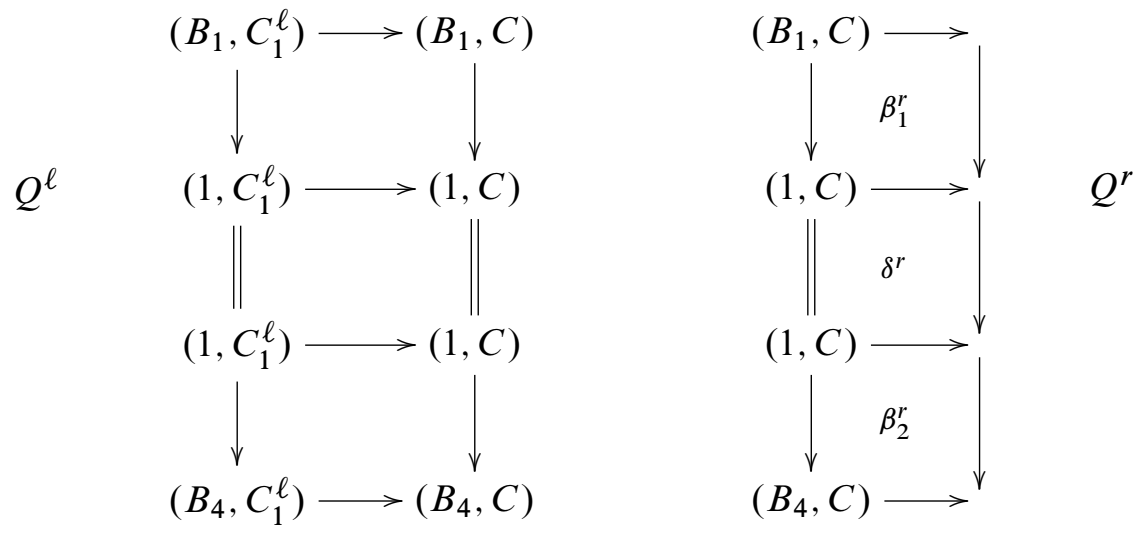

Figure 10: $\beta^{\ell}$ subdivided and $k^{r}=\mathrm{id}_{(1, C)}^{v}$
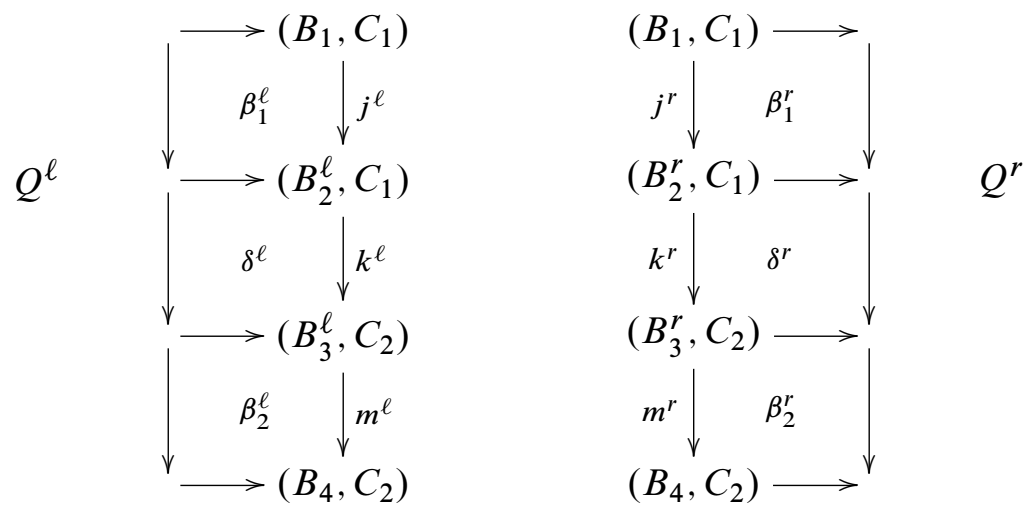

Figure 11: $Q^{\ell}$ and $Q^{r}$ both of form (2) and $\beta_{1}^{\ell}, \beta_{2}^{\ell}, \beta_{1}^{r}, \beta_{2}^{r}$ in $\mathbf{B} \otimes \mathbf{C}$

Figure 11. Then $B_{2}^{\ell}=1=B_{2}^{r}$ and $B_{3}^{\ell}=1=B_{3}^{r}$, since the only objects of $\mathbf{B} \otimes \mathbf{C}$ that are identified with an object of $\mathbb{D}$ are of the form $(1, C)$. Thus $j^{\ell}=j^{r}$ and $m^{\ell}=m^{r}$, as there is a unique vertical morphism from any object of $\mathbf{B} \otimes \mathbf{C}$ to another. Since $j^{\ell}$ and $m^{\ell}$ are invertible and $m^{\ell} k^{\ell} j^{\ell}=m^{\ell} k^{r} j^{\ell}$, we see also that $k^{\ell}=k^{r}$. Hence $Q^{\ell}$ and $Q^{r}$ can be horizontally composed to obtain a compatible arrangement equivalent to (1) or (2). 
Next we consider the case where $Q^{\ell}$ and $Q^{r}$ are both equivalent to compatible arrangements of the form (2), but the squares $\beta_{1}^{\ell}, \beta_{2}^{\ell}, \beta_{1}^{r}, \beta_{2}^{r}$ may be vertical identity squares in $\mathbb{P}$, that is, not necessarily in $\mathbf{B} \otimes \mathbf{C}$, as in Figure 12. Suppose $\beta_{1}^{\ell}$ is a vertical
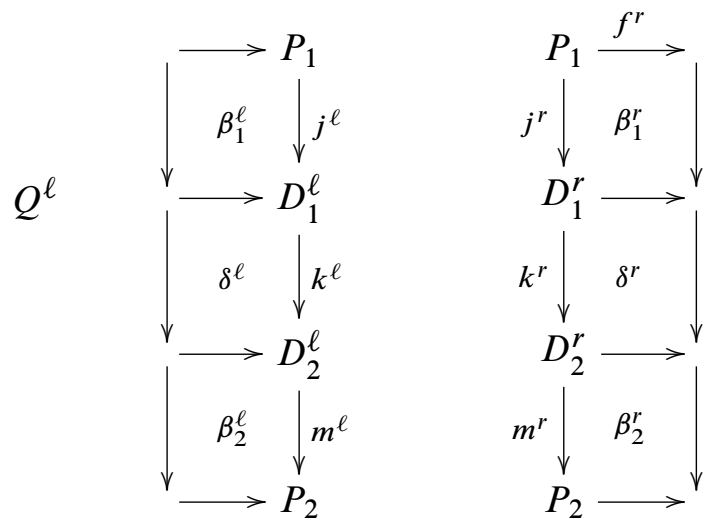

Figure 12: $Q^{\ell}$ and $Q^{r}$ both of form (2) and one of $\beta_{1}^{\ell}, \beta_{2}^{\ell}, \beta_{1}^{r}, \beta_{2}^{r}$ not in $\mathbf{B} \otimes \mathbf{C}$

identity square. Then $P_{1}=D_{1}^{\ell}$. We claim that $\beta_{1}^{r}$ is also a vertical identity square; there are two cases to prove. If $D_{1}^{\ell}$ is an object of $\mathbb{D}$ that is not of the form $(1, C)$, then $f^{r}$ cannot be in $\mathbf{B} \otimes \mathbf{C}$ (as its source is not in $\mathbf{B} \otimes \mathbf{C}$ ). Hence $\beta_{1}^{r}$ is a vertical identity square. For the second case, if $D_{1}^{\ell}$ is of the form $(1, C)$, then $j^{r}$ is a vertical arrow in $\mathbf{B} \otimes \mathbf{C}$ with source and target $(1, C)$. By the special form of squares in $\mathbf{B} \otimes \mathbf{C}$ in Figure 8 , we see that $\beta_{1}^{r}$ is also a vertical identity square. Thus, we have proved, if $\beta_{1}^{\ell}$ is a vertical identity square, then $\beta_{1}^{r}$ is also a vertical identity square. One can similarly show that if any one of $\beta_{1}^{\ell}, \beta_{2}^{\ell}, \beta_{1}^{r}, \beta_{2}^{r}$ is a vertical identity square, then the square next to it is also.

Let us continue the case where $Q^{\ell}$ and $Q^{r}$ are both equivalent to compatible arrangements of the form (2) as in Figure 12, and suppose again that $\beta_{1}^{\ell}$ is a vertical identity square. Then $\beta_{1}^{r}$ is also a vertical identity square. If either of $\beta_{2}^{\ell}$ or $\beta_{2}^{r}$ is a vertical identity square, then so is the other, in which case $Q^{\ell}$ and $Q^{r}$ can be horizontally composed to give a compatible arrangement equivalent to one of the form (1) or (2). If neither $\beta_{2}^{\ell}$ nor $\beta_{2}^{r}$ is a vertical identity square, then they are both in $\mathbf{B} \otimes \mathbf{C}$, and we can argue as in Figure 11 to conclude $D_{2}^{\ell}=D_{2}^{r}, m^{\ell}=m^{r}$, and $k^{\ell}=k^{r}$, in which case $Q^{\ell}$ and $Q^{r}$ can be horizontally composed to give a compatible arrangement equivalent to one of the form (1) or (2). 
The other cases of Figure 12, where one or more of $\beta_{1}^{\ell}, \beta_{2}^{\ell}, \beta_{1}^{r}, \beta_{2}^{r}$ is a vertical identity square in $\mathbb{P}$, are similar.

Therefore every square of $\mathbb{P}$ is equivalent to a compatible arrangement of the form (1) or (2), for both inclusions $i$ under consideration. This completes the proof of Theorem 10.6.

The two inclusions of Theorem 10.6 have some features in common, and the theorem holds for an entire class of inclusions $i: \mathbf{A} \longrightarrow \mathbf{B}$. We will return to the this topic and its interaction with Dawson-Paré-Pronk [24] in the future. Theorem 10.6 allows us to characterize the behavior of the horizontal nerve on such pushouts in Theorem 10.7, which we need to transfer the model structures from $\mathbf{C a t}^{\Delta^{\mathrm{op}}}$ in Section 7.

Theorem 10.7 Let $i: \mathbf{A} \longrightarrow \mathbf{B}$ be either of the following full inclusions.

$$
c \mathrm{Sd}^{2} \Lambda^{k}[m] \longrightarrow c \mathrm{Sd}^{2} \Delta[m]
$$

$\{1\} \longrightarrow \mathbf{I}$

Let $\mathbf{C}$ be a finite ordinal $[n]$ viewed as a category, $\mathbb{D}$ a double category, and $\mathbb{P}$ the pushout

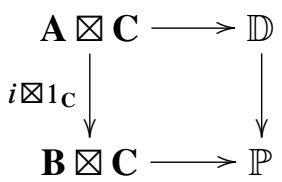

in DblCat. Then the induced map

$$
N_{h}(\mathbb{D}) \coprod_{N_{h}(\mathbf{A} \otimes \mathbf{C})} N_{h}(\mathbf{B} \otimes \mathbf{C}) \longrightarrow N_{h}(\mathbb{P})
$$

is an isomorphism of simplicial objects in Cat.

Proof We calculate the pushout

$$
N_{h}(\mathbb{D}) \coprod_{N_{h}(\mathbf{A} \otimes \mathbf{C})} N_{h}(\mathbf{B} \otimes \mathbf{C})
$$

levelwise and compare it with $N_{h}(\mathbb{P})$, whose form is known from Theorem 10.6. The horizontal nerve of an external product of categories is known from Proposition 5.8. In level 0 , the pushout (26) is

$$
\mathbb{D}_{0} \coprod_{\mathbf{A} \times(\mathrm{Obj} \mathbf{C})_{\text {disc }}} \mathbf{B} \times(\mathrm{Obj} \mathbf{C})_{\text {disc }}
$$


which is the same as the vertical 1 -category of $\mathbb{P}$ and thus $\left(N_{h} \mathbb{P}\right)_{0}$.

In level $k \geq 1$ the pushout (26) is

$$
N_{h}(\mathbb{D})_{k} \coprod_{\mathbf{A} \times N \mathbf{C}_{k}} \mathbf{B} \times N \mathbf{C}_{k}
$$

by Proposition 5.8. An application of Lemma 10.5 to level $k$ together with Theorem 10.6 give immediately that

$$
N_{h}(\mathbb{D})_{k} \coprod_{\mathbf{A} \times N \mathbf{C}_{k}}\left(\mathbf{B} \times N \mathbf{C}_{k}\right) \longrightarrow\left(N_{h} \mathbb{P}\right)_{k}
$$

is full (and is the identity on objects). To see that this functor is also faithful, we only need to concern ourselves with the squares of the form (2) in Theorem 10.6. For the Thomason structure these squares always have a representative where $\beta_{2}$ is of the form

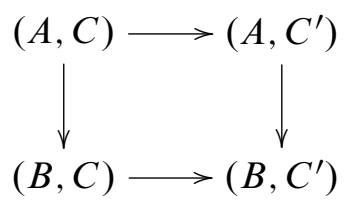

such that $A$ is a maximal element of $c \operatorname{Sd}^{2} \Lambda^{k}[n]$. This determines $\beta_{2}$ and $\delta$ uniquely. For the categorical structure all nonidentity $\beta_{2}$ squares are of the form

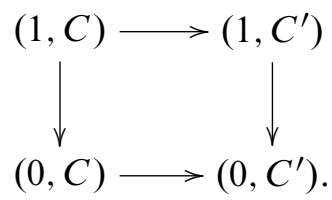

A similar argument for $\beta_{1}$ shows that $\beta_{1}, \delta$, and $\beta_{2}$ are determined uniquely.

\section{References}

[1] J Adámek, J Rosický, Locally presentable and accessible categories, London Math. Society Lecture Note Ser. 189, Cambridge University Press (1994) MR1294136

[2] A Bastiani, C Ehresmann, Multiple functors. I. Limits relative to double categories, Cah. Top. Géom. Différ. Catég. 15 (1974) 215-292 MR0379626

[3] M A Bednarczyk, A M Borzyszkowski, W Pawlowski, Generalized congruencesepimorphisms in Cat, Theory Appl. Categ. 5 (1999) No. 11, 266-280 MR1725510

[4] J Bénabou, Introduction to bicategories, from: "Reports of the Midwest Category Seminar", Springer, Berlin (1967) 1-77 MR0220789 
[5] J E Bergner, A survey of $(\infty, 1)$-categories, to appear in "Proceedings of the IMA Workshop ' $n$-Categories: Foundations and Applications' June 2004, University of Minnesota" arXiv:math/0610239

[6] J E Bergner, A model category structure on the category of simplicial categories, Trans. Amer. Math. Soc. 359 (2007) 2043-2058 MR2276611

[7] J E Bergner, Three models for the homotopy theory of homotopy theories, Topology 46 (2007) 397-436 MR2321038

[8] R Blackwell, G M Kelly, A J Power, Two-dimensional monad theory, J. Pure Appl. Algebra 59 (1989) 1-41 MR1007911

[9] R Boerger, Kongruenzrelationen auf Kategorien, Master's thesis, Westfälische Wilhelms-Universität Münster (1977)

[10] F Borceux, Handbook of categorical algebra. 1, Encyclopedia of Math. and its Applications 50, Cambridge University Press (1994) MR1291599 Basic category theory

[11] R Brown, P J Higgins, The equivalence of $\infty$-groupoids and crossed complexes, Cah. Top. Géom. Différ. 22 (1981) 371-386 MR639048

[12] R Brown, P J Higgins, The equivalence of $\omega$-groupoids and cubical T-complexes, Cah. Top. Géom. Différ. 22 (1981) 349-370 MR639047

[13] R Brown, P J Higgins, On the algebra of cubes, J. Pure Appl. Algebra 21 (1981) 233-260 MR617135

[14] R Brown, P J Higgins, Tensor products and homotopies for $\omega$-groupoids and crossed complexes, J. Pure Appl. Algebra 47 (1987) 1-33 MR906402

[15] R Brown, I Içen, Towards a 2-dimensional notion of holonomy, Adv. Math. 178 (2003) 141-175 MR1994346

[16] R Brown, K C H Mackenzie, Determination of a double Lie groupoid by its core diagram, J. Pure Appl. Algebra 80 (1992) 237-272 MR1170713

[17] R Brown, G H Mosa, Double categories, 2-categories, thin structures and connections, Theory Appl. Categ. 5 (1999) 163-175 MR1694653

[18] R Brown, C B Spencer, Double groupoids and crossed modules, Cah. Top. Géom. Différ. Catég. 17 (1976) 343-362 MR0440553

[19] M Bunge, R Paré, Stacks and equivalence of indexed categories, Cah. Top. Géom. Différ. 20 (1979) 373-399 MR558105

[20] R J M Dawson, R Paré, Characterizing tileorders, Order 10 (1993) 111-128 MR1253709

[21] R J M Dawson, R Paré, General associativity and general composition for double categories, Cah. Top. Géom. Différ. Catég. 34 (1993) 57-79 MR1213297

[22] R J M Dawson, R Paré, What is a free double category like?, J. Pure Appl. Algebra 168 (2002) 19-34 MR1879928 
[23] R J M Dawson, R Paré, D A Pronk, The structure of spans, preprint

[24] R J M Dawson, R Paré, D A Pronk, Free extensions of double categories, Cah. Topol. Géom. Différ. Catég. 45 (2004) 35-80 MR2040662

[25] R J M Dawson, R Paré, D A Pronk, Paths in double categories, Theory Appl. Categ. 16 (2006) No. 18, 460-521 MR2259260

[26] E J Dubuc, Kan extensions in enriched category theory, Lecture Notes in Math. 145, Springer, Berlin (1970) MR0280560

[27] A Ehresmann, C Ehresmann, Multiple functors. II. The monoidal closed category of multiple categories, Cah. Top. Géom. Différ. 19 (1978) 295-333 MR546074

[28] A Ehresmann, C Ehresmann, Multiple functors. III. The Cartesian closed category $\mathrm{Cat}_{n}$, Cah. Top. Géom. Différ. 19 (1978) 387-443 MR515164

[29] A Ehresmann, C Ehresmann, Multiple functors. IV. Monoidal closed structures on $\mathrm{Cat}_{n}$, Cah. Top. Géom. Différ. 20 (1979) 59-104 MR544529

[30] C Ehresmann, Catégories structurées, Ann. Sci. École Norm. Sup. (3) 80 (1963) 349-426 MR0197529

[31] C Ehresmann, Catégories et structures, Dunod, Paris (1965) MR0213410

[32] T Everaert, R W Kieboom, T Van der Linden, Model structures for homotopy of internal categories, Theory Appl. Categ. 15 (2005/06) No. 3, 66-94 MR2210576

[33] T M Fiore, Pseudo limits, biadjoints, and pseudo algebras: categorical foundations of conformal field theory, Mem. Amer. Math. Soc. 182 (2006) x+171 MR2229946

[34] T M Fiore, Pseudo algebras and pseudo double categories, J. Homotopy Relat. Struct. 2 (2007) 119-170 MR2369164

[35] T M Fiore, S Paoli, A Thomason model structure on the category of small $n$-fold categories arXiv:0808.4108

[36] R Fritsch, D M Latch, Homotopy inverses for nerve, Bull. Amer. Math. Soc. (N.S.) 1 (1979) 258-262 MR513754

[37] P Gabriel, M Zisman, Calculus of fractions and homotopy theory, Ergebnisse der Math. und ihrer Grenzgebiete 35, Springer, New York (1967) MR0210125

[38] R Garner, Double clubs, Cah. Topol. Géom. Différ. Catég. 47 (2006) 261-317 MR2303000

[39] PG Goerss, J F Jardine, Simplicial homotopy theory, Progress in Math. 174, Birkhäuser Verlag, Basel (1999) MR1711612

[40] M Grandis, Higher cospans and weak cubical categories (cospans in algebraic topology. I), Theory Appl. Categ. 18 (2007) No. 12, 321-347 MR2326435

[41] M Grandis, Cubical cospans and higher cobordisms (cospans in algebraic topology. III), J. Homotopy Relat. Struct. 3 (2008) 273-308 
[42] M Grandis, R Paré, Limits in double categories, Cah. Top. Géom. Différ. Catég. 40 (1999) 162-220 MR1716779

[43] M Grandis, R Paré, Adjoint for double categories. Addenda to: "Limits in double categories” [Cah. Topol. Géom. Différ. Catég. 40 (1999) 162-220; MR1716779], Cah. Topol. Géom. Différ. Catég. 45 (2004) 193-240 MR2090335

[44] M Grandis, R Paré, Lax Kan extensions for double categories (on weak double categories. IV), Cah. Topol. Géom. Différ. Catég. 48 (2007) 163-199 MR2351267

[45] M Grandis, R Paré, Kan extensions in double categories (on weak double categories. III), Theory Appl. Categ. 20 (2008) No. 8, 152-185 MR2395245

[46] PS Hirschhorn, Model categories and their localizations, Math. Surveys and Monogr. 99, Amer. Math. Soc. (2003) MR1944041

[47] M Hovey, Model categories, Math. Surveys and Monogr. 63, Amer. Math. Soc. (1999) MR1650134

[48] J R Isbell, Some remarks concerning categories and subspaces, Canad. J. Math. 9 (1957) 563-577 MR0094405

[49] M Johnson, The combinatorics of n-categorical pasting, J. Pure Appl. Algebra 62 (1989) 211-225 MR1026875

[50] A Joyal, R Street, Pullbacks equivalent to pseudopullbacks, Cah. Top. Géom. Différ. Catég. 34 (1993) 153-156 MR1223657

[51] A Joyal, M Tierney, Strong stacks and classifying spaces, from: "Category theory (Como, 1990)", Lecture Notes in Math. 1488, Springer, Berlin (1991) 213-236 MR1173014

[52] A Joyal, M Tierney, Quasi-categories vs Segal spaces, from: "Categories in algebra, geometry and mathematical physics”, Contemp. Math. 431, Amer. Math. Soc. (2007) 277-326 MR2342834

[53] G M Kelly, Elementary observations on 2-categorical limits, Bull. Austral. Math. Soc. 39 (1989) 301-317 MR998024

[54] G M Kelly, Basic concepts of enriched category theory, Repr. Theory Appl. Categ. (2005) vi+137 pp. MR2177301 Reprint of the 1982 original [Cambridge Univ. Press; MR0651714]

[55] T Kerler, V V Lyubashenko, Non-semisimple topological quantum field theories for 3-manifolds with corners, Lecture Notes in Math. 1765, Springer, Berlin (2001) MR1862634

[56] J Kock, Note on commutativity in double semigroups and two-fold monoidal categories, J. Homotopy Relat. Struct. 2 (2007) 217-228 MR2369167

[57] S Lack, A 2-categories companion, to appear in "Proceedings of the IMA Workshop ' $n$-Categories: Foundations and Applications' June 2004, University of Minnesota" arXiv:math/0702535 
[58] S Lack, A Quillen model structure for 2-categories, K-Theory 26 (2002) 171-205 MR1931220

[59] S Lack, A Quillen model structure for bicategories, K-Theory 33 (2004) 185-197 MR2138540

[60] S Lack, Homotopy-theoretic aspects of 2-monads, J. Homotopy Relat. Struct. 2 (2007) 229-260 MR2369168

[61] S Lack, S Paoli, An operadic approach to internal structures, Appl. Categ. Structures 13 (2005) 205-222 MR2167790

[62] S Lack, S Paoli, 2-nerves for bicategories, K-Theory 38 (2008) 153-175 MR2366560

[63] J-L Loday, Spaces with finitely many nontrivial homotopy groups, J. Pure Appl. Algebra 24 (1982) 179-202 MR651845

[64] S Mac Lane, Categories for the working mathematician, second edition, Graduate Texts in Math. 5, Springer, New York (1998) MR1712872

[65] S Mac Lane, I Moerdijk, Sheaves in geometry and logic, Universitext, Springer, New York (1994) MR1300636 A first introduction to topos theory, Corrected reprint of the 1992 edition

[66] J P May, J Sigurdsson, Parametrized homotopy theory, Math. Surveys and Monogr. 132, Amer. Math. Soc. (2006) MR2271789

[67] J Mersch, Structures quotients, Bull. Soc. Roy. Sci. Liege 33 (1964) 45-58 MR0178040

[68] J Mersch, Le problème du quotient dans les catégories, Mém. Soc. Roy. Sci. Liège Coll. in-8 (5) 11 (1965) 103 MR0213414

[69] J Morton, A double bicategory of cobordisms with corners arXiv:0611930

[70] S Paoli, Internal categorical structures in homotopical algebra, to appear in "Proceedings of the IMA Workshop ' $n$-Categories: Foundations and Applications' June 2004, University of Minnesota" Available at http://www.maths.mq.edu.au/ simonap/

[71] S Paoli, Semistrict models of connected 3-types and Tamsamani's weak 3-groupoids, J. Pure Appl. Algebra 211 (2007) 801-820 MR2344230

[72] A J Power, A 2-categorical pasting theorem, J. Algebra 129 (1990) 439-445 MR1040947

[73] A J Power, An n-categorical pasting theorem, from: "Category theory (Como, 1990)", Lecture Notes in Math. 1488, Springer, Berlin (1991) 326-358 MR1173022

[74] C Rezk, A model category for categories (2000) Available at http:// www.math.uiuc.edu/ rezk/cat-ho.dvi 
[75] C Rezk, A model for the homotopy theory of homotopy theory, Trans. Amer. Math. Soc. 353 (2001) 973-1007 MR1804411

[76] M Shulman, Comparing composites of left and right derived functors arXiv: 0706.2868

[77] M Shulman, Framed bicategories and monoidal fibrations arXiv:0706.1286

[78] R Street, Categorical structures, from: "Handbook of algebra, Vol. 1", North-Holland, Amsterdam (1996) 529-577 MR1421811

[79] A Strøm, The homotopy category is a homotopy category, Arch. Math. (Basel) 23 (1972) 435-441 MR0321082

[80] R W Thomason, Cat as a closed model category, Cah. Top. Géom. Différ. 21 (1980) 305-324 MR591388

[81] B Toën, Vers une axiomatisation de la théorie des catégories supérieures, $K$-Theory 34 (2005) 233-263 MR2182378

[82] V Trnková, Sum of categories with amalgamated subcategory, Comment. Math. Univ. Carolinae 6 (1965) 449-474 MR0190208

[83] K Worytkiewicz, K Hess, P E Parent, A Tonks, A model structure à la Thomason on 2-Cat MR2269840

Department of Mathematics, University of Chicago

5734 South University, Chicago, IL 60637, USA

and Departament de Matemàtiques, Universitat Autònoma de Barcelona

08193 Bellaterra (Barcelona), Spain

Department of Mathematics, Macquarie University

NSW 2109, Australia

Chase Building, Department of Mathematics and Statistics, Dalhousie University Halifax, Nova Scotia, Canada B3H 3J5, Canada

fiore@math.uchicago.edu, simonap@ics.mq.edu.au, pronk@mathstat.dal.ca http://www.math.uchicago.edu/ fiore/, http://www.ics.mq.edu.au/ simonap/, http://www.mathstat.dal.ca/Faculty/pronk.htm

Received: 26 October $2007 \quad$ Revised: 27 August 2008 